\title{
Functional-Analytic and Numerical Issues in Splitting Methods for Total Variation-based Image Reconstruction $\ddagger$
}

\author{
Michael Hintermüller \\ Department of Mathematics, Humboldt-Universität zu Berlin, Unter den Linden \\ 6, 10099 Berlin, Germany. \\ E-mail: hint@math.hu-berlin.de
}

\section{Carlos N. Rautenberg}

Department of Mathematics and Scientific Computing, Karl-Franzens-University of Graz, Heinrichstrasse 36, A-8010 Graz, Austria.

E-mail: carlos.rautenberg@uni-graz.at

\section{Jooyoung Hahn}

Part of the research for this work was performed while J.H. was a member of the SFB F32 04-N18 "Mathematical Optimization and Its Applications in Biomedical Sciences" at the Karl-Franzens-University of Graz. Current affiliation: AVL, Alte Poststrasse 152, 8010, Graz, Austria.

E-mail: jooyounghahn@gmail.com

\begin{abstract}
Variable splitting schemes for the function space version of the image reconstruction problem with total variation regularization (TV-problem) in its primal and pre-dual formulations are considered. In the primal splitting formulation, while existence of a solution cannot be guaranteed, it is shown that quasi-minimizers of the penalized problem are asymptotically related to the solution of the original TV-problem. On the other hand, for the pre-dual formulation a family of parametrized problems is introduced and a parameter dependent contraction of an associated fixed point iteration is established. Moreover, the theory is validated by numerical tests. Additionally, the augmented Lagrangian approach is studied, details on an implementation on a staggered grid are provided and numerical tests are shown.
\end{abstract}

$\ddagger$ This work was supported by the Austrian Ministry of Science and Education and the Austrian Science Fund FWF through START-Project Y305 Interfaces and Free Boundaries, and the SFB Project F32 04-N18 "Mathematical Optimization and Its Applications in Biomedical Sciences," the German Research Foundation DFG Research Center MATHEON through Projects C28, and the DFG Priority Program SPP 1253 through project Elliptic Mathematical Programs with Equilibrium Constraints (MPECs) in Function Space: Optimality Conditions and Numerical Realization. 


\section{Introduction}

Variable splitting and associated alternating algorithms have been recently successfully applied to discrete models in image reconstruction and denoising based on total variation (TV) regularization; see for example, [10,15,32,41-43]. The basic idea consists in the introduction of one or more new variables, a penalized problem and the resolution of two (or more) minimization problems significantly simpler than the original one. Algorithmically, this results in a sequence of nested iterations, which, on the discrete level, may exhibit an (image) resolution dependent convergence. As the penalty parameter is increased, one nevertheless expects that the solution of the original problem is approached. While this expectation may be justified in finite dimensions, in infinite dimensions new difficulties arise which render the existence of solutions of various sub-problems and the passage to the limit with the pertinent penalty parameter as delicate matters.

To the best of our knowledge, infinite dimensional analysis of such splitting and alternating minimization approaches in the appropriate Banach space setting have not yet been considered in the context of the TV-problem. Typically, rather the finite dimensional problem is studied in the literature (see [14], for example). The work by Setzer in [36] comes closest to the aforementioned function space analysis. However, it is confined to problems in Hilbert space settings only, whereas the TVproblem requires the non-reflexive space of functions of bounded variations as the associated solution space. In general, the study of infinite dimensional versions of variable splitting and other alternating minimization algorithms is motivated (among other reasons) by the possibility of obtaining mesh-independent numerical solution schemes upon discretization.

In the present paper, we are interested in studying splitting and associated alternating direction methods in function space; more precisely in the original nonreflexive Banach space associated with the TV-problem. In this context we consider the primal as well as the pre-dual formulations of the problem, and we study associated splitting techniques. Interestingly, it turns out that the existence of a solution to the associated penalized primal problem cannot be guaranteed, in general. Rather $\epsilon$ minimizers in the sense of Ekeland's variational principle can be assured only. For the penalized pre-dual problem, however, solutions do exist. As a consequence, the Bregman iteration or augmented Lagrangian schemes are not well defined in the primal context but can be shown to converge when applied to solving the pre-dual problem. We emphasize that, upon discretization, such distinct features get lost.

The pre-dual problem is posed in the Hilbert space $H_{0}(\operatorname{div})$, which is the space of $\mathbf{L}^{2}$-vector fields whose divergence is an element of $L^{2}(\Omega)$ as well; here $\Omega$ represents the image domain. For this reason we suggest a discretization of the penalized (and associated) pre-dual problem on staggered grids. It is shown that this approach compares favorably to energy based discretization techniques such as the one in [9].

In order to understand the difficulties associated when moving from finite dimensional into infinite dimensional spaces, it is useful to consider the following. In finite dimensions, the primal formulation of the total variation based denoising problem reads:

$$
\min \sum_{i} \frac{1}{2}\left|u_{i}-f_{i}\right|^{2}+\alpha\left|\left(\nabla_{h} u\right)_{i}\right|_{\ell^{2}} \quad \text { over } u \in \mathbb{R}^{N \times N},
$$

where $\nabla_{h}$ is a discrete gradient, $|\cdot|_{\ell^{2}}$ is the $\ell^{2}$-norm in $\mathbb{R}^{2}, \alpha>0$ is the regularization 
parameter, and $f \in \mathbb{R}^{N \times N}$ is a given (possibly noisy) image. In this problem, for the application of the an alternating minimization scheme, a new variable $\mathbf{p}=\left(p^{1}, p^{2}\right)$, with $p^{1}, p^{2} \in \mathbb{R}^{N \times N}$, is introduced and the following penalized problem is considered:

$\min \sum_{i} \frac{1}{2}\left|u_{i}-f_{i}\right|^{2}+\alpha\left|\mathbf{p}_{i}\right|_{\ell^{2}}+\frac{r}{2}\left|\left(\nabla_{h} u\right)_{i}-\mathbf{p}_{i}\right|_{\ell^{2}}^{2} \quad$ over $u \in \mathbb{R}^{N \times N}, \mathbf{p} \in \mathbb{R}^{N \times N} \times \mathbb{R}^{N \times N}$,

where $r>0$ denotes the penalty parameter and $\mathbf{p}_{i}=\left(p_{i}^{1}, p_{i}^{2}\right)$. For large $r>0$, it can be shown that this problem is related to (1): Let $r_{n} \rightarrow \infty$ and $\left(u^{n}, \mathbf{p}^{n}\right)$ be a solution to (2) for $r=r_{n}>0$ (note that the existence of solutions for (2) is guaranteed by standard methods). Since the problem is coercive, we have $\left(u^{n}, \mathbf{p}^{n}\right) \rightarrow\left(u^{*}, \mathbf{p}^{*}\right)$, along a subsequence still denoted by $\left\{\left(u^{n}, \mathbf{p}^{n}\right)\right\}$, for some $\left(u^{*}, \mathbf{p}^{*}\right)$ and also $\nabla_{h} u^{n} \rightarrow \nabla_{h} u^{*}$ along the previously picked subsequence. Given that $\left(u^{n}, \mathbf{p}^{n}\right)$ is a minimizer for (2), it follows that $r_{n} \sum_{i}\left|\left(\nabla_{h} u^{n}\right)_{i}-\mathbf{p}_{i}^{n}\right|_{\ell^{2}}^{2} \leq \sum_{i}\left|f_{i}\right|^{2}$ as $(u, \mathbf{p})=(0,0)$ is feasible for (2), which implies that $\nabla_{h} u^{n} \rightarrow \mathbf{p}^{*}=\nabla_{h} u^{*}$ for $r_{n} \rightarrow \infty$. Furthermore, by standard penalty arguments, one obtains $r_{n} \sum_{i}\left|\left(\nabla_{h} u^{n}\right)_{i}-\mathbf{p}_{i}^{n}\right|_{\ell^{2}}^{2} \rightarrow 0$ and that $\left(u^{*}, \mathbf{p}^{*}\right)$ is a minimizer of (1).

The infinite dimensional analogue to the TV-problem is given by

$$
\min \frac{1}{2} \int_{\Omega}|u-f|^{2}+\alpha|\mathcal{D} u|(\Omega) \quad \text { over } u \in B V(\Omega),
$$

where $|\mathcal{D} u|(\Omega)$ is the total mass of the Borel measure $\mathcal{D} u$ determined by the distributional derivative of $u$, and $f \in L^{2}(\Omega)$ is given. If we follow the same procedure as in the finite dimensional case, we need to introduce a new variable $\mathbf{p}$ in the same space as $\mathcal{D} u$, that is, $\mathbf{p}$ should be a Borel measure. As a consequence, the penalty term $\frac{r}{2}|\mathcal{D} u-\mathbf{p}|_{L^{2}}^{2}$ appears no longer appropriate, since neither $\mathbf{p}$ nor $\mathcal{D} u$ need to belong to $L^{2}(\Omega)$ and might have no pointwise description over $\Omega$. Ignoring this fact and nevertheless adding $\frac{r}{2}|\mathcal{D} u-\mathbf{p}|_{L^{2}}^{2}$ to the objective in (3) with $|\mathcal{D} u|(\Omega)$ replaced by $|\mathbf{p}|_{\mathbf{L}^{1}}$ significantly changes the nature of the problem. As a consequence, existence of a solution $\left(u_{r}^{*}, \mathbf{p}_{r}^{*}\right)$ to the resulting penalized problem cannot be guaranteed, in general. Furthermore, in case the penalty term is taken to be $\frac{r}{2}|\mathcal{D} u-\mathbf{p}|(\Omega)$, then, although the problem is now well-defined as we prove in section 2.1, there are no advantages in the splitting approach over the original TV-formulation. This discussion is continued in section 2.1 below where a solution to this issue is also proposed. In the case of the pre-dual formulation, similar (yet more amenable) challenges are faced and solved in section 3.1.

In section 2 we are concerned with a variable splitting approach for the primal formulation of the TV-problem. We provide an infinite dimensional penalty framework based on $\epsilon$-minimizers in a sufficient regular state space which is amenable to numerical implementation. Under appropriate conditions it is proven that the sequence of $\epsilon$ minimizers converges to the solution of (3). Furthermore, provided regularity and boundedness assumptions hold true, we show that an augmented Lagrangian-looking penalty functional exhibits the same behavior than the pure penalty method.

Section 3 is devoted to the variable splitting approach for the Fenchel pre-dual formulation of the TV-problem. It is proven (Lemma 3.1) that the sequence of minimizers of the associated penalized problem is well-defined and converges (in a certain sense) to the solution of the pre-dual problem. Additionally, we show that it is necessary to analyze the alternating algorithm within a different framework than the standard backward-backward or forward-backward schemes; see, e.g., [30]. It is also 
shown that an augmented Lagrangian approach is suitable for the infinite dimensional problem, in the sense that there exists a Lagrange multiplier such that the solution of the splitting problem for the pre-dual formulation is a global minimizer of the augmented Lagrangian, without the necessity of a limiting process for the penalty parameter.

Details of a numerical implementation on a staggered grid for the pre-dual formulation are provided in Section 4. Our numerical evidence shows that the finite difference scheme on a staggered grid has advantages with respect to other existing approaches in the literature; specifically, it is shown to reduce direction dependent blurriness in reconstructions. We also show compatibility properties of the discrete divergence and gradient operators and provide details on the numerical implementation of the algorithms used throughout the paper on a staggered grid. The section ends with comparative results obtained by the algorithms specified in the paper and in the existing literature.

Notation. Let $\Omega \subset \mathbb{R}^{2}$ be a bounded and open domain. We denote by $L^{p}(\Omega)$ the usual Lebesgue space of real-valued functions on the domain $\Omega$. The space $\mathbf{L}^{p}(\Omega)$ is defined as $L^{p}(\Omega) \times L^{p}(\Omega)$ such that $\mathbf{f}:=\left(f_{1}, f_{2}\right): \Omega \rightarrow \mathbb{R}^{2}$ belongs to $\mathbf{L}^{p}(\Omega)$ iff $f_{i} \in L^{p}(\Omega)$ for $i=1,2$. The Sobolev space $W^{1,1}(\Omega)$ contains weakly differentiable functions in $L^{1}(\Omega)$ whose weak derivatives also belong to $\mathbf{L}^{1}(\Omega)$ (see [1] for a definition of the Sobolev space). It is endowed with the norm $|v|_{W^{1,1}}=|v|_{L^{1}}+|\nabla v|_{\mathbf{L}^{1}}$. Throughout the paper, strong convergence is denoted by " $\rightarrow$ " and weak convergence by "-". For a vector $\mathbf{x} \in \mathbb{R}^{2}$, the Euclidian norm is denoted either by $|\mathbf{x}|_{\ell^{2}}$ or $|\mathbf{x}|$.

The Lebesgue measure of a measurable set $\Omega$ is denoted as $|\Omega|$, and we say that a property holds "a.e. in $\Omega$ ", if it is true in $\Omega$ except for a subset $\Omega_{0} \subset \Omega$ such that $\left|\Omega_{0}\right|=0$.

We denote by $\mathscr{L}(V, W)$ the vector space of bounded linear operators between the normed vector spaces $V$ and $W$ endowed with the norm $|G|_{\mathscr{L}(V, W)}=\sup _{|v|_{V}=1}|G v|_{W}$, for $G \in \mathscr{L}(V, W)$.

The following results can be found in [3], which we also follow closely notationwise. We denote the set of $\mathbb{R}^{2}$-valued Borel measures by $\mathbf{M}\left(\Omega ; \mathbb{R}^{2}\right)$. If $B$ is a Borel set, then then total mass $|\mu|(B)$ for $\mu \in \mathbf{M}\left(\Omega ; \mathbb{R}^{2}\right)$ is defined as

$$
|\mu|(B):=\sup \left\{\sum_{i=0}^{\infty}\left|\mu\left(B_{i}\right)\right|: \bigcup_{i=0}^{\infty} B_{i}=B\right\},
$$

where the supremum is taken over all partitions of $B$ in its Borel field. Note that $\mathbf{M}\left(\Omega ; \mathbb{R}^{2}\right)$ is a Banach space when endowed with the norm $\mu \mapsto|\mu|(\Omega)$. Additionally, if $\mu \in \mathbf{M}\left(\Omega ; \mathbb{R}^{2}\right)$, then $|\mu|$ is a non-negative Borel measure and $|\mu|(B)=\int_{B} \mathrm{~d}|\mu|$.

The space of functions of bounded variation over $\Omega$ is denoted as $B V(\Omega)$ and it is defined to be the space of functions $u \in L^{1}(\Omega)$ with gradient $\mathcal{D} u$ (in the distributional sense) in $\mathbf{M}\left(\Omega ; \mathbb{R}^{2}\right)$. Endowed with the norm

$$
|u|_{B V}=|u|_{L^{1}}+|\mathcal{D} u|(\Omega),
$$

$B V(\Omega)$ becomes a Banach space, and we write $\int_{\Omega}|\mathcal{D} u|:=|\mathcal{D} u|(\Omega)$ (in order to avoid the notation $\left.\int_{\Omega} \mathrm{d}|\mathcal{D} u|\right)$. Note that if $u \in W^{1,1}(\Omega)$, then $u \in B V(\Omega)$ and $|\mathcal{D} u|(\Omega)=\int_{\Omega}|\nabla u| \mathrm{d} x$, where $\nabla u$ refers to the weak gradient of $u$. It can be proven that

$$
\int_{\Omega}|\mathcal{D} u|=\sup \left\{\left.\int_{\Omega} u \operatorname{divvd} x\left|\mathbf{v} \in C_{c}^{1}\left(\Omega ; \mathbb{R}^{2}\right),\right| \mathbf{v}(\mathbf{x})\right|_{\ell^{2}} \leq 1 \text { a.e. } \mathbf{x} \in \Omega\right\},
$$


where $C_{c}^{1}\left(\Omega ; \mathbb{R}^{2}\right)$ denotes the set of continuously differentiable functions $u: \Omega \rightarrow \mathbb{R}^{2}$ with compact support in $\Omega$.

\section{The primal formulation}

This section is devoted to the study of a continuous analogue to the splitting and penalty methods in [41] for the minimization of a discrete TV- problem . Let $\Omega \subset \mathbb{R}^{2}$ be an open, bounded image domain with Lipschitz continuous boundary $\partial \Omega$, and $f \in L^{2}(\Omega)$ is a given noisy image. The continuous primal formulation of the TV-problem (also called ROF-problem due to the seminal work by Rudin, Osher and Fatemi in [35]) is given by

$$
\bar{u}=\underset{u \in B V(\Omega)}{\arg \min }\left\{\mathcal{J}_{p}(u):=\frac{1}{2} \int_{\Omega}|u-f|^{2}+\alpha|\mathcal{D} u|(\Omega)\right\},
$$

where $\alpha$ is a positive constant.

In many papers (see for instance $[10,32,35,41,42]$ ) finite dimensional versions of (5) are considered. In this context, splitting techniques introducing a new variable and penalizing (by means of the $\ell_{2}$-norm) deviations of this new variable from being the gradient of the reconstructed image are proposed. Such a procedure circumvents the difficulties arising from the nonlinearity and the non-differentiability of the regularization term. Moreover, the (local) convergence analysis of associated iteration schemes for the numerical solution benefit from the discrete context, but may lead to resolution-/mesh-dependent convergence. In the context of (5), however, a straightforward extension to $L^{2}(\Omega)$-penalization (rather than the discrete $\ell_{2}$-penalty) appears no longer suitable as $\mathcal{D} u$ is a measure only. Furthermore, the non-reflexivity of $B V(\Omega)$ challenges the existence of a solution of the reformulation of (5) relevant in variable splitting approaches.

\subsection{A primal variable splitting method}

In [41], a primal variable splitting approach in finite dimensions was considered. In this section we want to understand whether this approach can be related to the original problem (5) and an associated variable splitting technique relying on $L^{2}(\Omega)$ penalties. In fact, the function space analogue of the associated subproblem in the variable splitting approach reads

$$
\min \mathcal{E}_{n}^{p}(u, \mathbf{p}):=\frac{1}{2} \int_{\Omega}|u-f|^{2}+\alpha \int_{\Omega}|\mathbf{p}|+\frac{r_{n}}{2}|\mathbf{p}-\nabla u|_{\mathbf{L}^{2}}^{2}, \quad \text { over }(u, \mathbf{p}) \in X,
$$

where $f \in L^{2}(\Omega)$, and for each $n \in \mathbb{N}$ we have $\mathcal{E}_{n}^{p}: X \rightarrow \mathbb{R} \cup\{+\infty\}$ with $X:=W^{1,1}(\Omega) \times \mathbf{L}^{1}(\Omega), r_{n}>0$ for $n \in \mathbb{N}$ and $r_{n} \rightarrow+\infty$ for $n \rightarrow+\infty$. Since $\mathcal{E}_{n}^{p}$ is not coercive and $X$ is not reflexive, existence of a solution to (6) cannot be guaranteed for fixed $n$. As a remedy, we rather study the behavior of a sequence $\left\{\left(u_{n}, \mathbf{p}_{n}\right)\right\}$ in $X$ associated with $r_{n} \uparrow+\infty$. For this purpose, let $\left(u_{n}, \mathbf{p}_{n}\right)$ satisfy

$$
\mathcal{E}_{n}^{p}\left(u_{n}, \mathbf{p}_{n}\right) \leq \inf _{(u, \mathbf{p}) \in X} \mathcal{E}_{n}^{p}(u, \mathbf{p})+\epsilon_{n},
$$

for $\epsilon_{n}>0, n \in \mathbb{N}$, with $\epsilon_{n} \downarrow 0$. 
Let us briefly comment on the choice of $X$ in this context. First of all, $W^{1,1}(\Omega)$ appears somewhat minimal for $(6)$ and it is continuously embedded in $B V(\Omega)$. For $u \in W^{1,1}(\Omega)$ we have $\nabla u \in \mathbf{L}^{1}(\Omega)$ and $|\mathcal{D} u|(\Omega)=\int_{\Omega}|\nabla u| d x$. We note that other penalized unconstrained problems are conceivable for the splitting approach. A particular example of a suitable objective is

$$
B V(\Omega) \times \mathbf{M}\left(\Omega ; \mathbb{R}^{2}\right) \ni(u, \mathbf{p}) \mapsto \frac{1}{2} \int_{\Omega}|u-f|^{2}+\alpha|\mathbf{p}|(\Omega)+r_{n}|\mathbf{p}-\mathcal{D} u|(\Omega) .
$$

In this case, minimizers do exist but the minimization problem is essentially at least as complicated as the original TV-problem. Concerning the existence of a solution to (8), let $\left\{\left(u_{k}, \mathbf{p}_{k}\right)\right\}$ be an infimizing sequence for (8). Then $\left|\mathbf{p}_{k}\right|(\Omega)<\infty$, and hence there exists $\mathbf{p}^{*} \in \mathbf{M}\left(\Omega ; \mathbb{R}^{2}\right)$ such that $\mathbf{p}_{k} \rightarrow \mathbf{p}^{*}$ (along a subsequence) in the $\sigma\left(C_{c}^{\prime}, C_{c}\right)$ topology, where $C_{c}$ and $C_{c}^{\prime}$ stand for $C_{c}\left(\Omega ; \mathbb{R}^{2}\right)$ and its dual, respectively. Therefore, we have that $\left|\mathbf{p}^{*}\right|(\Omega) \leq \underline{\lim }_{k \rightarrow \infty}\left|\mathbf{p}_{k}\right|(\Omega)$. Additionally, $\left\{u_{k}\right\}$ is bounded in $L^{2}(\Omega)$ (and hence in $L^{1}(\Omega)$ ) and $\left\{\mathcal{D} u_{k}\right\}$ is bounded in $\mathbf{M}\left(\Omega ; \mathbb{R}^{2}\right)$ (by the boundedness of $\left\{\mathbf{p}_{k}\right\}$ ), from which we infer that $\left\{u_{k}\right\}$ is bounded in $B V(\Omega)$. This implies that there exists $u^{*} \in B V(\Omega)$ such that $u_{k} \rightarrow u^{*}$ in $L^{2}(\Omega)$ and $\mathcal{D} u_{k} \rightarrow \mathcal{D} u^{*}$ in the $\sigma\left(C_{c}^{\prime}, C_{c}\right)$-topology along a suitable subsequence; see Proposition 10.1.1. in [3] and Theorem 10.1.4., in the same reference, for a proof of the compact embedding $B V(\Omega) \hookrightarrow L^{1}(\Omega)$. Therefore, we have

$$
\begin{aligned}
\frac{1}{2} \int_{\Omega}\left|u^{*}-f\right|^{2}+\alpha\left|\mathbf{p}^{*}\right|(\Omega)+ & r_{n}\left|\mathbf{p}^{*}-\mathcal{D} u^{*}\right|(\Omega) \leq \\
& \varliminf_{k \rightarrow \infty}\left[\frac{1}{2} \int_{\Omega}\left|u_{k}-f\right|^{2}+\alpha\left|\mathbf{p}_{k}\right|(\Omega)+r_{n}\left|\mathbf{p}_{k}-\mathcal{D} u_{k}\right|(\Omega)\right],
\end{aligned}
$$

i.e., $\left\{\left(u^{*}, \mathbf{p}^{*}\right)\right\}$ is a minimizer of (8). Additionally, a similar argument can be used to show that the sequence of minimizers $\left\{\left(u_{n}^{*}, \mathbf{p}_{n}^{*}\right)\right\}$ of (8) satisfies $u_{n}^{*} \rightarrow u^{* *}$ in $L^{2}(\Omega)$, $\mathbf{p}_{n} \rightarrow \mathcal{D} u^{* *}$ in the $\sigma\left(C_{c}^{\prime}, C_{c}\right)$ - topology, both along a subsequence as $r_{n} \rightarrow \infty$, where $u^{* *} \in B V(\Omega)$ solves the TV-problem. However, as noted before, in this variable splitting scheme we have no advantages (neither theoretical nor numerical) over the original TV-formulation as one still needs to handle the TV-seminorm $|\cdot|(\Omega)$.

Now, we return to (5) and show that the minimization problem is associated with asymptotic properties of the functional (6). The proof of the following result is given in Appendix A.

Theorem 2.1 Let $\left\{\left(u_{n}, \mathbf{p}_{n}\right)\right\}$ be the sequence in $X$ which is defined by (7). Then, we have

$$
u_{n} \rightarrow \bar{u} \text { in } L^{2}(\Omega) \text { and } \int_{\Omega}\left|\mathbf{p}_{n}\right| \rightarrow|\mathcal{D} \bar{u}|(\Omega)
$$

along a subsequence as $n \rightarrow \infty$, where $\bar{u} \in B V(\Omega)$ is given by (5).

Note that the penalized problem (6) can be formally related to the constrained optimization problem

$$
\begin{aligned}
& \min \mathcal{J}_{p}(u, \mathbf{p}):=\frac{1}{2}|u-f|_{L^{2}}^{2}+\alpha|\mathbf{p}|_{\mathbf{L}^{1}} \quad \text { over }(u, \mathbf{p}) \in X, \\
& \text { subject to (s.t.) } g(u, \mathbf{p}):=\nabla u-\mathbf{p}=0 .
\end{aligned}
$$


In finite dimensions, augmented Lagrangian methods have been applied successfully for solving image reconstruction problems over the recent years; see for example $[10,25,42]$. However, in the infinite dimensional context difficulties arise, not only of theoretical nature but also associated with the numerical implementation of the algorithm. For example, the functional $X \ni(u, \mathbf{p}) \mapsto \mathcal{J}_{p}(u, \mathbf{p})$ is not differentiable and Lagrange multipliers associated with the constraint (11) might not exist. In order to overcome these issues, the following theorem considers a "pseudo augmented Lagrangian" setting, where the pseudo-Lagrange multipliers are regular objects.

For this purpose consider the sequences $\left\{r_{n}\right\}$ in $\mathbb{R}^{+}$with $r_{n+1} \geq r_{n}$ and $\lim _{n \rightarrow \infty} r_{n} \rightarrow \infty$ and $\left\{\boldsymbol{\lambda}_{n}\right\}$ in $\mathbf{L}^{2}(\Omega)$ with $\sup _{n \in \mathbb{N}}\left|\boldsymbol{\lambda}_{n}\right|_{\mathbf{L}^{2}}<\infty$. For each $n \in \mathbb{N}$, we define the extended real-valued functional $\mathcal{L}_{r_{n}}^{p}: X \times \mathbf{L}^{2}(\Omega) \rightarrow \mathbb{R} \cup\{+\infty\}$ as

$$
\mathcal{L}_{r_{n}}^{p}\left(u, \mathbf{p}, \boldsymbol{\lambda}_{n}\right):=\frac{1}{2}|u-f|_{L^{2}}^{2}+\alpha|\mathbf{p}|_{\mathbf{L}^{1}}+\frac{r_{n}}{2}|\mathbf{p}-\nabla u|_{\mathbf{L}^{2}}^{2}+\int_{\Omega} \boldsymbol{\lambda}_{n} \cdot(\mathbf{p}-\nabla u) .
$$

Similarly as before, we consider a sequence $\left\{\left(u_{n}, \mathbf{p}_{n}\right)\right\}$ in $X$ of $\epsilon$-minimizers of $(u, \mathbf{p}) \mapsto \mathcal{L}_{r_{n}}^{p}(u, \mathbf{p}, \boldsymbol{\lambda})$. Let $\left\{\epsilon_{n}\right\}$ satisfy $\epsilon_{n}>0$ for all $n \in \mathbb{N}$ and $\epsilon_{n} \downarrow 0$. Moreover, for a fixed $n \in \mathbb{N}$ let $\left(u_{n}, \mathbf{p}_{n}\right)$ satisfy

$$
\mathcal{L}_{r_{n}}^{p}\left(u_{n}, \mathbf{p}_{n}, \boldsymbol{\lambda}_{n}\right) \leq \inf _{(u, \mathbf{p}) \in X} \mathcal{L}_{r_{n}}^{p}\left(u, \mathbf{p}, \boldsymbol{\lambda}_{n}\right)+\epsilon_{n}
$$

We observe that the sequence $\left\{\left(u_{n}, \mathbf{p}_{n}\right)\right\}$ is well-defined since $(u, \mathbf{p}) \mapsto \mathcal{L}_{r_{n}}^{p}\left(u, \mathbf{p}, \boldsymbol{\lambda}_{n}\right)$ is bounded from below and, thus, admits an infimum over $X$. For the sake of brevity suppose that $r_{n} \geq 1$. Then, completing squares in the last two terms of $\mathcal{L}_{r_{n}}^{p}$, we obtain $\mathcal{L}_{r_{n}}^{p}\left(u, \mathbf{p}, \boldsymbol{\lambda}_{n}\right) \geq-\left|\boldsymbol{\lambda}_{n}\right|_{\mathbf{L}^{2}} \geq-\sup _{n \in \mathbb{N}}\left|\boldsymbol{\lambda}_{n}\right|_{\mathbf{L}^{2}}>-\infty$. We are now in a position to state (its proof is deferred to the Appendix A) the analogue to Theorem 2.1 but for the functional $\mathcal{L}_{r_{n}}^{p}$.

Theorem 2.2 Let $\left\{\left(u_{n}, \mathbf{p}_{n}\right)\right\}$ be the sequence defined by (13). Then the assertion of Theorem 2.1 holds true.

2.1.1. The Discrete Augmented Lagrangian For the sake of keeping this paper selfcontained, we briefly specify the augmented Lagrangian method for solving a discrete version of (10)-(11). For details see, e.g., [32,42]. Given a penalty parameter $r_{n}>0$ and an (approximate) multiplier $\boldsymbol{\lambda}$, the discrete augmented Lagrangian reads

$$
\begin{gathered}
\mathcal{L}_{n, h}^{p}(u, \mathbf{p}, \boldsymbol{\lambda}):=h^{2} \sum_{(i, j) \in \Omega_{h}} \frac{1}{2}\left|u_{i, j}-f_{i, j}\right|^{2}+\alpha\left|\mathbf{p}_{i, j}\right|+\frac{r_{n}}{2}\left|\mathbf{p}_{i, j}-\left(\nabla_{h} u\right)_{i, j}\right|^{2} \\
+\boldsymbol{\lambda}_{i, j} \cdot\left(\mathbf{p}_{i, j}-\left(\nabla_{h} u\right)_{i, j}\right)
\end{gathered}
$$

where $\mathcal{L}_{n, h}^{p}: X_{h} \rightarrow \mathbb{R} \cup\{\infty\}$ with $X_{h}:=\mathbb{R}^{N^{2}} \times \mathbb{R}^{2 N^{2}} \times \mathbb{R}^{2 N^{2}}$. Note that for the ease of exposition we assume that the discrete image domain $\Omega_{h}$ consists of $N^{2}=N \times N$ pixels denoted by $(i, j)$, which are uniformly distributed. Fixing $\Omega=(0,1)^{2}$, this implies that the (equi)distance between two neighbouring pixels is $h=\frac{1}{N-1}$. Further, $\nabla_{h}$ denotes a difference approximation of $\nabla$; see, e.g., [8] for a suitable one-sided difference approximation, which we need to weight by $1 / h$ to be consistent with the scaling by $h$. The operator $-\operatorname{div}_{h}$ represents the adjoint of $\nabla_{h}$.

In Algorithm 1, an alternating minimization scheme (see $[32,42]$ ) for the augmented Lagrangian is implemented. For a fixed penalty parameter $r_{n}$, "suitable 


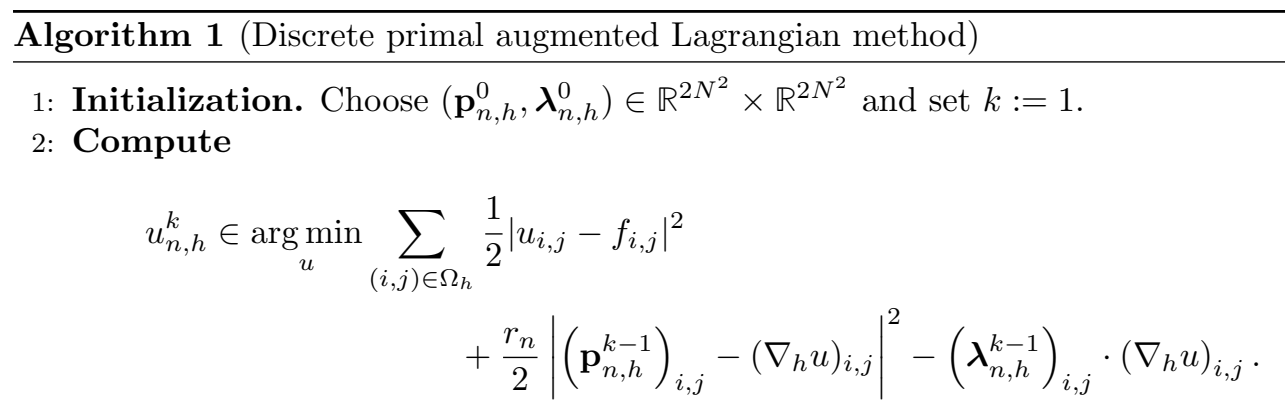

3: Compute

$$
\mathbf{p}_{n, h}^{k} \in \underset{\mathbf{p}}{\arg \min } \sum_{(i, j) \in \Omega_{h}} \alpha\left|\mathbf{p}_{i, j}\right|+\frac{r_{n}}{2}\left|\mathbf{p}_{i, j}-\left(\nabla_{h} u_{n, h}^{k}\right)_{i, j}\right|^{2}+\left(\boldsymbol{\lambda}_{n, h}^{k-1}\right)_{i, j} \cdot \mathbf{p}_{i, j} .
$$

4: Set

$$
\left(\boldsymbol{\lambda}_{n, h}^{k}\right)_{i, j}:=\left(\boldsymbol{\lambda}_{n, h}^{k-1}\right)_{i, j}+r_{n}\left(\left(\mathbf{p}_{n, h}^{k}\right)_{i, j}-\left(\nabla_{h} u_{n, h}^{k}\right)_{i, j}\right), \quad(i, j) \in \Omega_{h}
$$

5: Check stopping criteria. If suitable stopping criteria are met, $\left(u_{n, h}^{*}, \mathbf{p}_{n, h}^{*}, \boldsymbol{\lambda}_{n, h}^{*}\right)=\left(u_{n, h}^{k}, \mathbf{p}_{n, h}^{k}, \boldsymbol{\lambda}_{n, h}^{k}\right) ;$ otherwise set $k:=k+1$ and return to step 2 .

stopping criteria" in step 5 of Algorithm 1 refer to the norm of the residual of the first-order optimality conditions dropping below a user-specified tolerance. In step 3 of Algorithm 1, a soft-thresholding technique provides an explicit solution which guarantees the first-order optimality condition of the $\mathbf{p}$-subproblem. Thus, it suffices to check whether the residual of the first-order optimality condition of the $u$-subproblem in step 2, i.e.,

$$
\mathbf{R}_{n, h}^{k}:=r_{n} \operatorname{div}_{h}\left(\mathbf{p}_{n, h}^{k}-\nabla_{h} u_{n, h}^{k}\right)+\operatorname{div}_{h} \boldsymbol{\lambda}_{n, h}^{k}+u_{n, h}^{k}-f,
$$

sufficiently small in norm.

It is known (see [42] for a proof) that for any finite positive penalty parameter $r_{n}>0$, a sequence $\left\{\left(u_{n, h}^{k}, \mathbf{p}_{n, h}^{k}, \boldsymbol{\lambda}_{n, h}^{k}\right)\right\}_{k \in \mathbb{N}}$ (generated by Algorithm 1) converges to a saddle point of $\mathcal{L}_{n, h}^{p}(u, \mathbf{p}, \boldsymbol{\lambda})$, as $k \rightarrow \infty$. Moreover the limit point of $\left\{u_{n, h}^{k}\right\}$ is a minimizer of the discrete ROF/TV-functional. In addition, the algorithm is equivalent to the split Bregman method; see [21]. However, it is not clear whether one can obtain the same convergence results in infinite dimensions. In fact, the update in step 2 of Algorithm 1 might no longer be appropriate, as the Lagrange multiplier, if it exists at all, might not allow for pointwise descriptions. A similar issue with respect to the multiplier is also present in step 3. Moreover, the update rule in step 4 has to be considered with care in order to reflect the (function space) regularity of the involved quantities.

This concludes our analysis and numerical development for the primal problem. 


\section{The Fenchel pre-dual formulation}

It has been observed that a (pre)dual version of the TV-problem (5) are numerically more amenable than its primal counterpart; see $[8,11,23,24]$. In this section, we therefore propose splitting algorithms for finding a solution of the TVproblem (5) from a solution to its Fenchel pre-dual formulation:

$$
\overline{\mathbf{s}} \in \underset{\mathbf{s} \in H_{0}(\operatorname{div})}{\arg \min }\left\{\mathcal{J}_{d}(\mathbf{s}):=\frac{1}{2}|\operatorname{divs}+f|_{L^{2}}^{2}: \quad|\mathbf{s}|_{\ell^{2}} \leq \alpha \text { a.e. in } \Omega\right\},
$$

where $\operatorname{div} \in \mathscr{L}\left(H_{0}(\operatorname{div}), L^{2}(\Omega)\right)$, with

$$
H_{0}(\operatorname{div})=\left\{\mathbf{s} \in \mathbf{L}^{2}(\Omega): \operatorname{divs} \in L^{2}(\Omega), \mathbf{s} \cdot \nu=0 \text { on } \partial \Omega\right\}
$$

and $\nu$ the outward unit normal vector on $\partial \Omega$. For more information on (16) and associated solvers see [23] and [13]. A solution of the TV-problem (5) is obtained from $\overline{\mathbf{s}}$ by

$$
\bar{u}=\operatorname{div} \overline{\mathbf{s}}+f \quad \text { in } \quad \Omega .
$$

A main feature of the minimization problem (16) is that a minimizer need not be unique due to the non-trivial kernel of the div-operator. In [23] an orthogonal projection in $\mathbf{L}^{2}(\Omega)$ onto $\left\{\mathbf{s} \in H_{0}(\operatorname{div})\right.$ : divs $=0$ a.e. in $\left.\Omega\right\}$ is used in order to obtain a unique solution in (16). In [24] a Tikhonov-type regularization in $H_{0}^{1}(\Omega)$ is considered as a remedy against non-uniqueness. In the aforementioned paper it is shown that the Tikhonov regularization in the pre-dual formulation (16) changes the TV-regularization in the primal formulation (5) into a local Huber-type regularization.

As announced above, in recent years algorithms operating on the (pre-)dual formulation of the TV-problem have become popular; see, e.g., [8, 23, 24]. While in finite dimensions, the dual problem equals the pre-dual, this is not the case in infinite dimensions as a consequence of the non-reflexivity of $B V(\Omega)$.

The primal-dual algorithms in $[23,24]$ in the infinite dimensional setting exhibit local superlinear convergence. In order to obtain a solution of the TV- problem, the Tikhonov-regularization parameters contained in these approaches need to vanish; otherwise the iterates of the algorithms in $[23,24]$ remain in a space more regular than $B V(\Omega)$.

In finite dimensions, an algorithm for computing a minimizer of a discretized version of $\mathcal{J}_{d}$ in (16) is proposed in [8], where its conditional convergence is also proved. Moreover, concerning proper discretization of $\mathcal{J}_{d}$, in [9] an upwind finite-difference method is introduced and shown to reduce anisotropy of the reconstruction, when compared to standard schemes like the ones in [8]. However, it is not straightforward to extend the algorithms proposed in $[8,9]$ to infinite dimensional spaces.

\subsection{A variable splitting method}

In this and the next section we study (pre-)dual variable splitting and augmented Lagrangian methods in function space, respectively. For this purpose let $\mathcal{R} \subset \mathbf{L}^{2}(\Omega)$ be defined as

$$
\mathcal{R}:=\left\{\mathbf{t} \in \mathbf{L}^{2}(\Omega):|\mathbf{t}(\mathbf{x})| \leq \alpha \text { a.e. in } \Omega\right\},
$$


and let $\delta_{\mathcal{R}}$ denote the indicator function

$$
\delta_{\mathcal{R}}(\mathbf{t})= \begin{cases}0 & \mathbf{t} \in \mathcal{R}, \\ +\infty & \text { otherwise }\end{cases}
$$

Utilizing a variable splitting approach by introducing $\mathbf{t}$ and considering

$$
\min \mathcal{E}_{n}^{d}(\mathbf{s}, \mathbf{t}):=\frac{1}{2}|\operatorname{divs}+f|_{L^{2}}^{2}+\frac{q_{n}}{2}|\mathbf{s}-\mathbf{t}|_{L^{2}}^{2}+\delta_{\mathcal{R}}(\mathbf{t}) \quad \text { over }(\mathbf{s}, \mathbf{t}) \in Y,
$$

where $Y:=H_{0}(\operatorname{div}) \times \mathbf{L}^{2}(\Omega)$ and $\mathcal{E}_{n}^{d}: Y \rightarrow \mathbb{R} \cup\{+\infty\}$, for $q_{n}>0$ an approximate version of (16) is obtained. Before we commence our study of (20), its relation to (16) and its numerical realization, we discuss the current understanding of backwardbackward (BB) and forward-backward (FB) iterations for solving approximate versions of (16), we then relate those methods to (20) and our proposed iterative scheme, which is summarized in Algorithm 2 below. As a bottom line, we emphasize already here that typical (BB)- or (FB)-iterations for solving (16) do not guarantee a function space analysis, whereas our method does.

3.1.1. Backward-Backward and Forward-Backward schemes In this subsection we propose a splitting alternating minimization algorithm (Algorithm 2 below) to approximate solutions of (16). As the scheme can be written as a particular case of a generalized version of either a backward-backward or forward-backward algorithm (also called implicit-implicit and explicit-implicit scheme, respectively, see [30]). We first review such methods in a general setting.

Suppose that $A, B$ and $A+B$ are maximal monotone operators (see for example [4] for a definition of maximal monotone operator) over a Hilbert space $\mathscr{H}$, respectively, and $C$ is a linear bounded operator on the same Hilbert space. We are interested in the following abstract problem:

$$
\text { Find } \mathbf{s} \in \mathscr{H} \text {, such that } 0 \in(A+B)(\mathbf{s}) .
$$

The following algorithmic schemes are intended to solve (21): Given an initial guess $\mathbf{s}^{0} \in \mathscr{H}$, the backward-backward algorithm is given by

$$
\mathbf{s}^{k+1}=(I+\lambda A)^{-1} C(I+\lambda B)^{-1} \mathbf{s}^{k}, \quad k=0,1,2, \ldots
$$

and the forward-backward method by

$$
\mathbf{s}^{k+1} \in(I+\lambda A)^{-1} C(I-\lambda B) \mathbf{s}^{k}, \quad k=0,1,2, \ldots
$$

In the case $C=I,(\mathrm{BB})$ and (FB) determine the classical backward-backward and forward-backward algorithms, respectively. A general operator theoretic approach for proving convergence of these algorithms was taken by Lions and Mercier (see [30]). Specific applications of (FB) to variational inequalities can be found in $[5,6]$ and of (BB) in [29]. For applications of (FB) to general convex constrained minimization, the results trace back to the work of Goldstein (see [20]). Strong convergence of the sequence of iterates generated by (BB) could be obtained assuming that $B$ is coercive, and strong linear convergence is implied if $B$ is Lipschitz continuous and strongly monotone (see $[12,30]$ ). Although weak convergence may be obtained under weaker conditions, convergence to a zero of $A+B$ can only be guaranteed for weighted 
averages of $\left\{\mathbf{s}^{k}\right\}$ if $\lambda \geq 0$ is replaced by a sequence $\left\{\lambda_{k}\right\} \in \ell^{2} \backslash \ell^{1}$ (see [29] and [33]). Strong convergence of the sequence of iterates generated by (FB) depends on several conditions. In fact, one requires Lipschitz continuity of $B$, with either $A$ or $B$ strongly monotone and some extra hypothesis on $\lambda$ (see $[12,18]$ and [16] for a finite dimensional account of the issue).

In order to anticipate some of the subsequent development we mention already here, that in the case of our splitting algorithm, if we fit the iteration into either (BB) and (FB) with $C=I$, the space $\mathscr{H}$ can be taken as $H_{0}($ div $)$ or $\mathbf{L}^{2}(\Omega)$. In either scenario, we show that $A$ and/or $B$ do not satisfy the known conditions to provide strong convergence. Furthermore, even weak convergence may not be guaranteed as we show in section 3.1.3. We overcome these drawbacks by choosing $C \neq I$, providing different state spaces for each step and by relaxing restrictions on $A$ and $B$. Eventually, we prove strong linear convergence of the iteration to an approximate solution of (16) in Theorem 3.1.

3.1.2. Pre-dual splitting scheme. Now we return to (20) and show that minimizers of $\mathcal{E}_{n}^{d}$ are closely related to problem (16) for $n \rightarrow \infty$, provided that $\lim _{n \rightarrow \infty} q_{n}=\infty$.

Lemma 3.1 For each $n \in \mathbb{N}$, there exists a minimizer $\left(\mathbf{s}_{n}, \mathbf{t}_{n}\right)$ of $\mathcal{E}_{n}^{d}: H_{0}($ div $) \times$ $\mathbf{L}^{2}(\Omega) \rightarrow \mathbb{R}$. For $q_{n} \rightarrow \infty$ as $n \rightarrow \infty$, one further has $\mathbf{s}_{n} \rightarrow \overline{\mathbf{s}}$ (along a subsequence) in $H_{0}$ (div) as $n \rightarrow \infty$, where $\overline{\mathbf{s}}$ is a solution to (16).

Proof. If $\mathbf{t} \notin \mathcal{R}$, then $\mathcal{E}_{n}^{d}(\mathbf{s}, \mathbf{t})=\infty$. Hence, it suffices to consider the constrained minimization problem

$$
\min \mathcal{E}_{n}^{d}(\mathbf{s}, \mathbf{t}) \quad \text { over }(\mathbf{s}, \mathbf{t}) \in Y_{\mathcal{R}},
$$

where $Y_{\mathcal{R}}:=H_{0}(\operatorname{div}) \times \mathcal{R} \subset Y$.

We first note that $\mathcal{E}_{n}^{d}$ is convex and prove that it is coercive on $Y_{\mathcal{R}}$. For this purpose, suppose that the sequence $\left\{\left(\mathbf{s}_{\ell}, \mathbf{t}_{\ell}\right)\right\}$ in $Y_{\mathcal{R}}=H_{0}(\operatorname{div}) \times \mathcal{R} \subset Y$, with $\ell \in \mathbb{N}$, is unbounded. Since $\mathbf{t}_{\ell} \in \mathcal{R}$ and $\mathcal{R}$ is a bounded subset of $\mathbf{L}^{2}(\Omega)$ it follows that $\left\{\mathbf{t}_{\ell}\right\}$ is bounded and, thus, $\left\{\mathbf{s}_{\ell}\right\}$ is unbounded in $H_{0}$ (div), i.e., $\left|\mathbf{s}_{\ell}\right|_{\mathbf{L}^{2}} \rightarrow \infty$ or $\left|\operatorname{divs} \mathbf{s}_{\ell}\right|_{L^{2}} \rightarrow \infty$. The reverse triangle inequality yields

$$
\mathcal{E}_{n}^{d}(\mathbf{s}, \mathbf{t}) \geq \frac{1}{2}\left(|\operatorname{divs}|_{L^{2}}-|f|_{L^{2}}\right)^{2}+\frac{q_{n}}{2}\left(|\mathbf{s}|_{\mathbf{L}^{2}}-|\mathbf{t}|_{\mathbf{L}^{2}}\right)^{2},
$$

and hence $\mathcal{E}_{n}^{d}\left(\mathbf{s}_{\ell}, \mathbf{t}_{\ell}\right) \rightarrow \infty$ as $\ell \rightarrow \infty$, i.e., $\mathcal{E}_{n}^{d}$ is coercive over $Y_{\mathcal{R}}$.

It follows directly that $\mathcal{E}_{n}^{d}$ is continuous and convex. Moreover, since $Y_{\mathcal{R}} \subset Y$ is closed, $\mathcal{E}_{n}^{d}$ is weakly lower semicontinuous over $Y_{\mathcal{R}}$. Since $Y$ is reflexive, the problem in (22) has a solution and consequently, for each $n \in \mathbb{N}, \mathcal{E}_{n}^{d}$ has minimizers over $Y$.

For $n \in \mathbb{N}$, let $\left(\overline{\mathbf{s}}_{n}, \overline{\mathbf{t}}_{n}\right) \in Y$ be a minimizer of $\mathcal{E}_{n}^{d}$. Next we study $n \rightarrow \infty$ and relate limit points to (16).

For the sake of simplicity assume that $q_{n} \leq q_{n+1}$ for $n \in \mathbb{N}$, otherwise we can extract an increasing subsequence. Then, as $(0,0) \in Y_{\mathcal{R}}$, we infer $\frac{1}{2}|f|_{L^{2}}^{2}=$ $\mathcal{E}_{n}^{d}(0,0) \geq \mathcal{E}_{n}^{d}\left(\overline{\mathbf{s}}_{n}, \overline{\mathbf{t}}_{n}\right)$ and $\mathcal{E}_{n}^{d}\left(\overline{\mathbf{s}}_{n}, \overline{\mathbf{t}}_{n}\right) \leq \mathcal{E}_{n}^{d}\left(\overline{\mathbf{s}}_{n+1}, \overline{\mathbf{t}}_{n+1}\right) \leq \mathcal{E}_{n+1}^{d}\left(\overline{\mathbf{s}}_{n+1}, \overline{\mathbf{t}}_{n+1}\right)$ for all $n \in \mathbb{N}$. It follows from standard arguments for penalty functions (see [31]) that $\lim _{n \rightarrow \infty} \frac{q_{n}}{2}\left|\overline{\mathbf{s}}_{n}-\overline{\mathbf{t}}_{n}\right|_{\mathbf{L}^{2}}^{2}=0$.

Since $\left\{\overline{\mathbf{t}}_{n}\right\}$ in $\mathcal{R}$ is bounded in $\mathbf{L}^{2}(\Omega)$ and $\frac{1}{2}|f|_{L^{2}}^{2} \geq \mathcal{E}_{n}^{d}\left(\overline{\mathbf{s}}_{n}, \overline{\mathbf{t}}_{n}\right)$, from the coercivity of $\mathcal{E}_{n}^{d}$ we obtain that $\left\{\overline{\mathbf{s}}_{n}\right\}$ is bounded in $H_{0}($ div $)$. Thus, since $H_{0}(\operatorname{div}) \times \mathbf{L}^{2}(\Omega)$ is a Hilbert space, there exists a subsequence of $\left\{\left(\overline{\mathbf{s}}_{n}, \overline{\mathbf{t}}_{n}\right)\right\}$ (again denoted by $\left\{\left(\overline{\mathbf{s}}_{n}, \overline{\mathbf{t}}_{n}\right)\right\}$ ) 
for which $\left(\overline{\mathbf{s}}_{n}, \overline{\mathbf{t}}_{n}\right) \rightarrow(\overline{\mathbf{s}}, \overline{\mathbf{t}})$ in $H_{0}(\operatorname{div}) \times \mathbf{L}^{2}(\Omega)$ as $n \rightarrow \infty$. Now, let $\mathbf{s} \in \mathcal{R} \cap H_{0}(\operatorname{div})$ be arbitrary, but fixed. Then

$$
\frac{1}{2}|\operatorname{divs}+f|_{L^{2}}^{2}=\mathcal{E}_{n}^{d}(\mathbf{s}, \mathbf{s}) \geq \mathcal{E}_{n}^{d}\left(\overline{\mathbf{s}}_{n}, \overline{\mathbf{t}}_{n}\right) \geq \frac{1}{2}\left|\operatorname{div} \overline{\mathbf{s}}_{n}+f\right|_{L^{2}}^{2}+\frac{q_{n}}{2}\left|\overline{\mathbf{s}}_{n}-\overline{\mathbf{t}}_{n}\right|_{\mathbf{L}^{2}}^{2} .
$$

Using the weakly lower semicontinuity of the norm and $\lim _{n \rightarrow \infty} \frac{q_{n}}{2}\left|\overline{\mathbf{s}}_{n}-\overline{\mathbf{t}}_{n}\right|_{\mathbf{L}^{2}}^{2}=0$, we obtain

$$
\frac{1}{2}|\operatorname{divs}+f|_{L^{2}}^{2} \geq \frac{1}{2}|\operatorname{div} \overline{\mathbf{s}}+f|_{L^{2}}^{2} .
$$

As $\mathcal{R}$ is weakly closed, we have that $\overline{\mathbf{t}} \in \mathcal{R}$. This fact and $\lim _{n \rightarrow \infty}\left|\overline{\mathbf{s}}_{n}-\overline{\mathbf{t}}_{n}\right|_{\mathbf{L}^{2}}=0$ yield $\overline{\mathbf{s}} \in \mathcal{R} \cap H_{0}$ (div). Finally, as $\mathbf{s} \in \mathcal{R} \cap H_{0}$ (div) was arbitrary, $\overline{\mathbf{s}}$ is a solution to (16).

Remark. The previous theorem can be strengthened. In fact, besides $\mathbf{s}_{n}^{*} \rightarrow \mathbf{s}^{*}$ in $H_{0}(\operatorname{div})$, one also has divs ${ }_{n}^{*} \rightarrow \operatorname{divs} \mathbf{s}^{*}$ in $L^{2}(\Omega)$. However, whether $\mathbf{s}_{n}^{*} \rightarrow \mathbf{s}^{*}$ in $H_{0}(\operatorname{div})$, remains an open question.

In order to study the convergence of our splitting algorithm, we introduce the following auxiliary problems: For $0<\eta<1$ let $\mathcal{G}_{n}, \mathcal{G}_{n}^{\eta}: H_{0}$ (div) $\rightarrow \mathbb{R}$ be defined by $\mathcal{G}_{n}(\mathbf{s}):=\mathcal{E}_{n}^{d}\left(\mathbf{s}, P_{\mathcal{R}}(\mathbf{s})\right)$ and $\mathcal{G}_{n}^{\eta}(\mathbf{s}):=\mathcal{E}_{n}^{d}\left(\mathbf{s}, \eta P_{\mathcal{R}}(\mathbf{s})\right)$, where $P_{\mathcal{R}}(\mathbf{s})$ is the $\mathbf{L}^{2}(\Omega)$ projection of $\mathbf{s}$ onto $\mathcal{R}$, i.e.,

$$
\begin{aligned}
& \mathcal{G}_{n}(\mathbf{s})=\frac{1}{2}|\operatorname{divs}+f|_{L^{2}}^{2}+\frac{q_{n}}{2}\left|\mathbf{s}-P_{\mathcal{R}}(\mathbf{s})\right|_{\mathbf{L}^{2}}^{2} \\
& \mathcal{G}_{n}^{\eta}(\mathbf{s})=\frac{1}{2}|\operatorname{divs}+f|_{L^{2}}^{2}+\frac{q_{n}}{2}\left|\mathbf{s}-\eta P_{\mathcal{R}}(\mathbf{s})\right|_{\mathbf{L}^{2}}^{2}
\end{aligned}
$$

We utilize an alternating minimization algorithm (Algorithm 2) for approximating minimizers of (20). For this purpose and given some $\mathbf{t}_{\eta}^{k-1} \in \mathcal{R}$, we introduce a new family of problems, which depend on $\eta \in(0,1)$ :

$$
\mathbf{s}_{\eta}^{k}=\underset{\mathbf{s} \in H_{0}(\operatorname{div})}{\arg \min } \frac{1}{2}|\operatorname{divs}+f|_{L^{2}}^{2}+\frac{q}{2}\left|\mathbf{s}-\eta \mathbf{t}_{\eta}^{k-1}\right|_{\mathbf{L}^{2}}^{2} .
$$

The update for $\mathbf{t}_{\eta}^{k-1}$ is computed by $\mathbf{t}_{\eta}^{k}=P_{\mathcal{R}}\left(\mathbf{s}_{\eta}^{k}\right)$.

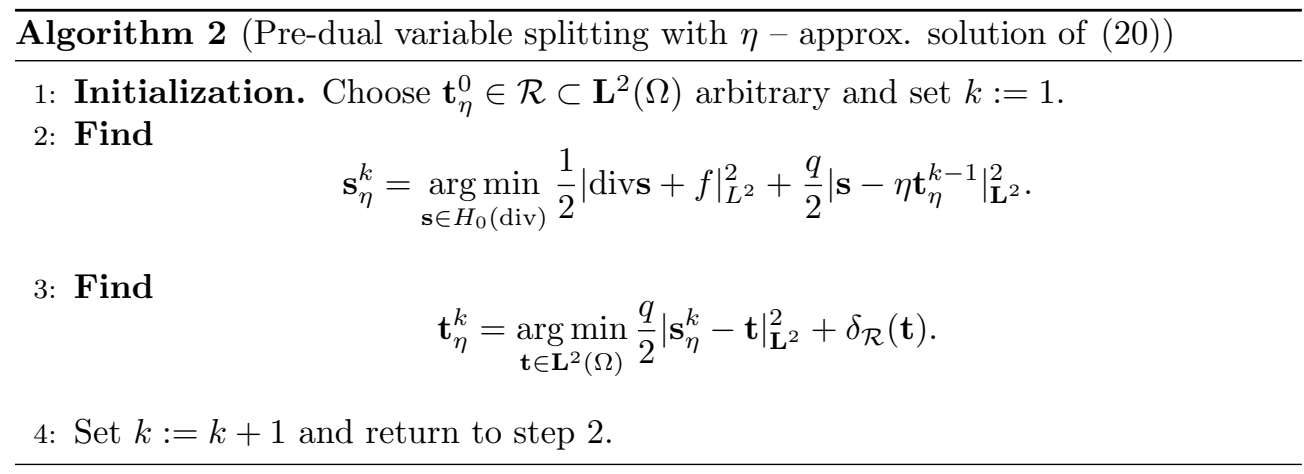

A few words on Algorithm 2 are in order. The introduction of the variable $\mathbf{t}$ in the functional $\mathcal{E}_{n}^{d}(\mathbf{s}, \mathbf{t})$ in $(20)$ helps to handle the pointwise inequality constraint in the numerical implementation. The alternating direction algorithm with the variable $\mathbf{t}$ 
divides the minimization problems for $\mathcal{E}_{n}^{d}(\mathbf{s}, \mathbf{t})$ in Algorithm 2 into two parts. Each subproblem has a unique solution and their respective numerical implementation is rather straightforward. In fact, the first-order optimality condition of the ssubproblem reduces to a linear elliptic partial differential equation of second order, and a projection provides an explicit solution for the $\mathbf{t}$-subproblem. The parameter $\eta \in(0,1)$ is necessary to guarantee convergence (in function space) of the sequence $\left\{\mathbf{s}_{\eta}^{k}\right\}$ to some $\mathbf{s}_{\eta} \in H_{0}(\operatorname{div})$ as $k \rightarrow \infty$ with $\mathbf{s}_{\eta}$ a minimizer of $\mathcal{G}_{n}^{\eta}$; see Theorem 3.1(i) below. Theorem 3.1(ii) asserts that a sequence $\left\{\mathbf{s}_{\eta_{i}}\right\}$ of minimizers of $\mathcal{G}_{n}^{\eta_{i}}(\cdot)$ converges to a minimizer of $\mathcal{G}_{n}(\cdot)$ as $\eta_{i} \uparrow 1$. A detailed discussion of Algorithm 2 for $\eta=1$ in view of the shortcomings of classical theory for backward-backward (BB) and forwardbackward (FB) iterations is included in section 3.1.3. Specifically, it is argued that already weak convergence can only be expected in special cases confined to $\mathbf{L}^{2}(\Omega)$ rather than $H_{0}$ (div), and strong convergence appears to be impossible by means of classical results in the literature.

The following theorem establishes properties of the iteration $\left\{\mathbf{s}_{\eta}^{k}\right\}_{k=1}^{\infty}$ in $H_{0}(\operatorname{div})$ such as the strong convergence of the sequence of iterates and relations to the minimizers of $\mathcal{G}_{n}$ and $\mathcal{G}_{n}^{\eta}$ as well as $\mathcal{E}_{n}^{d}$.

Theorem 3.1 Suppose that $n \in \mathbb{N}$ is fixed, and consider $H_{0}(\operatorname{div}) \times \mathbf{L}^{2}(\Omega) \ni(\mathbf{s}, \mathbf{t}) \mapsto$ $\mathcal{E}_{n}^{d}(\mathbf{s}, \mathbf{t}), H_{0}(\operatorname{div}) \ni \mathbf{s} \mapsto \mathcal{G}^{d}(\mathbf{s}), \mathcal{G}_{n}^{\eta}(\mathbf{s})$ as defined in (20) and (24), respectively, and the sequence $\left\{\mathbf{s}_{\eta}^{k}\right\}_{k=1}^{\infty}$ in $H_{0}$ (div) defined by Algorithm 2 with $q=q_{n}$. Then the following holds true:

i. For each $0<\eta<1$, there exists $\mathbf{s}_{\eta} \in H_{0}$ (div) such that $\mathbf{s}_{\eta}^{k} \rightarrow \mathbf{s}_{\eta}$ in $H_{0}$ (div) as $k \rightarrow \infty$, where the convergence is linear. The map $(0,1) \ni \eta \mapsto \mathbf{s}_{\eta} \in H_{0}$ (div) is locally Lipschitz continuous, and $\mathbf{s}_{\eta}$ is the unique minimizer of $\mathcal{G}_{n}^{\eta}$.

ii. If $\left\{\eta_{i}\right\}$ is a sequence in $(0,1)$ with $\eta_{i} \uparrow 1$ as $i \rightarrow \infty$, then the sequence $\left\{\mathbf{s}_{\eta_{i}}\right\}$ of minimizers of $\mathcal{G}_{n}^{\eta_{i}}$ satisfies $\mathbf{s}_{\eta_{i}} \rightarrow \mathbf{s}^{*}$ (along a subsequence) with $\mathbf{s}^{*} \in H_{0}(\operatorname{div})$, a minimizer of $\mathcal{G}_{n}$.

iii. The pair $\left(\mathbf{s}^{*}, P_{\mathcal{R}}\left(\mathbf{s}^{*}\right)\right) \in H_{0}(\operatorname{div}) \times \mathbf{L}^{2}(\Omega)$ is a minimizer of $\mathcal{E}_{n}^{d}$, i.e., it is a solution of $(20)$.

Proof. We start by proving i. Let $\mathbf{t} \in \mathbf{L}^{2}(\Omega)$ be fixed, and define $\mathcal{Q}(\mathbf{s}):=$ $\frac{1}{2}|\operatorname{divs}+f|_{L^{2}}^{2}+\frac{q}{2}|\mathbf{s}-\eta \mathbf{t}|_{\mathbf{L}^{2}}^{2}$ for $\mathbf{s} \in H_{0}($ div $)$. Then, $\mathcal{Q}$ is clearly continuous on $H_{0}(\operatorname{div})$ and the inequality

$$
\mathcal{Q}(\mathbf{s}) \geq \frac{1}{2}\left(|\operatorname{divs}|_{L^{2}}-|f|_{L^{2}}\right)^{2}+\frac{q}{2}\left(|\mathbf{s}|_{\mathbf{L}^{2}}-\eta|\mathbf{t}|_{\mathbf{L}^{2}}\right)^{2}
$$

implies that $\mathcal{Q}$ is coercive. Also, since the norms in $L^{2}(\Omega)$ and $\mathbf{L}^{2}(\Omega)$ are strictly convex and weakly lower semi-continuous, $\mathbf{s} \mapsto \mathcal{Q}(\mathbf{s})$ is strictly convex and weakly lower semicontinuous in $H_{0}$ (div) and, hence, admits a unique global minimizer. Thus, $\mathbf{s}_{\eta}^{k}$ is well-defined in step 2 of Algorithm 2 and we can write $\mathbf{s}_{\eta}^{k}=T\left(\eta \mathbf{t}_{\eta}^{k-1}\right)($ for $k \in \mathbb{N})$ where $T(\eta \cdot): \mathbf{L}^{2}(\Omega) \rightarrow H_{0}(\operatorname{div})$ denotes the solution map associated with the problem in step 2 . Since $\mathcal{R}$ is closed, convex and non-empty in $\mathbf{L}^{2}(\Omega)$, step 3 of Algorithm 2 is equivalent to the projection of $\mathbf{s}$ onto $\mathcal{R}$. As a consequence, $\mathbf{t}_{\eta}^{k}=P_{\mathcal{R}}\left(\mathbf{s}_{\eta}^{k}\right)$ is uniquely defined. Therefore, we can write $\mathbf{s}_{\eta}^{k}=T\left(\eta P_{\mathcal{R}}\left(\mathbf{s}_{\eta}^{k-1}\right)\right)$.

Next, consider $|\mathbf{s}|_{q}^{2}:=|\operatorname{divs}|_{L^{2}}^{2}+q|\mathbf{s}|_{\mathbf{L}^{2}}^{2}$ for $\mathbf{s} \in H_{0}($ div $)$, which defines a norm equivalent to $|\cdot|_{H_{0}(\mathrm{div})}$. We now prove that $T\left(\eta P_{\mathcal{R}}(\cdot)\right)$ is a contraction with respect 
to this norm. As the objective in step 2 of Algorithm 2 is Fréchet differentiable in $H_{0}$ (div), the first order optimality condition for $\mathbf{s}_{i}=T\left(\mathbf{t}_{i}\right)$ for $i=1,2$ reads

$$
\left(\operatorname{divs}_{i}+f, \operatorname{divh}_{i}\right)_{L^{2}}+q\left(\mathbf{s}_{i}-\mathbf{t}_{i}, \mathbf{h}_{i}\right)_{\mathbf{L}^{2}}=0, \quad \forall \mathbf{h}_{i} \in H_{0}(\operatorname{div}) .
$$

Taking $\mathbf{h}_{1}=\mathbf{h}_{2}=\mathbf{s}_{2}-\mathbf{s}_{1}$ above, and subtracting the equality for $i=2$ from the one for $i=1$, we obtain

$$
\left|\operatorname{div}\left(\mathbf{s}_{2}-\mathbf{s}_{1}\right)\right|_{L^{2}}^{2}+q\left|\mathbf{s}_{2}-\mathbf{s}_{1}\right|_{\mathbf{L}^{2}}^{2}=q\left(\mathbf{t}_{2}-\mathbf{t}_{1}, \mathbf{s}_{2}-\mathbf{s}_{1}\right)_{\mathbf{L}^{2}} .
$$

This yields $|\delta \mathbf{s}|_{q}^{2} \leq q|\delta \mathbf{t}|_{\mathbf{L}^{2}}|\delta \mathbf{s}|_{\mathbf{L}^{2}}$, with $\delta \mathbf{s}:=\mathbf{s}_{2}-\mathbf{s}_{1}$ and $\delta \mathbf{t}:=\mathbf{t}_{2}-\mathbf{t}_{1}$. This implies that $|\delta \mathbf{s}|_{q} \leq q^{1 / 2}|\delta \mathbf{t}|_{\mathbf{L}^{2}}$. Since $\mathbf{L}^{2}(\Omega)$ is a Hilbert space, we have $\left|P_{\mathcal{R}}\left(\mathbf{u}_{2}\right)-P_{\mathcal{R}}\left(\mathbf{u}_{1}\right)\right|_{\mathbf{L}^{2}} \leq$ $\left|\mathbf{u}_{2}-\mathbf{u}_{1}\right|_{\mathbf{L}^{2}}$ for all $\mathbf{u}_{\mathbf{1}}, \mathbf{u}_{\mathbf{2}} \in H_{0}(\operatorname{div}) \subset \mathbf{L}^{2}(\Omega)$. Then, for $\mathbf{t}_{i}=\eta P_{\mathcal{R}}\left(\mathbf{u}_{i}\right)$ we infer that $|\delta \mathbf{s}|_{q} \leq \eta q^{1 / 2}|\delta \mathbf{u}|_{\mathbf{L}^{2}} \leq \eta|\delta \mathbf{u}|_{q}$, i.e.,

$$
\left|T\left(\eta P_{\mathcal{R}}\left(\mathbf{u}_{2}\right)\right)-T\left(\eta P_{\mathcal{R}}\left(\mathbf{u}_{1}\right)\right)\right|_{q} \leq \eta\left|\mathbf{u}_{2}-\mathbf{u}_{1}\right|_{q}
$$

Since $0<\eta<1$, by the Banach Contraction Principle, the sequence $\mathbf{s}_{\eta}^{k}=$ $T\left(\eta P_{\mathcal{R}}\left(\mathbf{s}_{\eta}^{k-1}\right)\right)$ converges strongly to $\mathbf{s}_{\eta}$ in the $q$-norm and hence in the usual norm for $H_{0}$ (div), as well which is the unique fixed point for $H_{0}(\operatorname{div}) \ni \mathbf{s} \mapsto T\left(\eta P_{\mathcal{R}}(\mathbf{s})\right)$. This implies that $\mathbf{s}_{\eta}$ is the unique minimizer of $H_{0}(\operatorname{div}) \ni \mathbf{s} \mapsto \mathcal{G}_{n}^{\eta}(\mathbf{s})$ in (24). In fact, if there were other minimizers, then these minimizers would also be fixed points of $T\left(\eta P_{\mathcal{R}}(\cdot)\right)$, which contradict the above argument.

Above, we have shown that $|\delta \mathbf{s}|_{q} \leq q^{1 / 2}|\delta \mathbf{t}|_{\mathbf{L}^{2}}$ for $\mathbf{s}_{i}=T\left(\mathbf{t}_{i}\right), i=1,2$. Now, let $\eta_{1}, \eta_{2} \in(0,1)$. Hence there exist $\mathbf{s}_{\eta_{i}} \in H_{0}$ (div) such that $\mathbf{s}_{\eta_{i}}=T\left(\eta_{i} P_{\mathcal{R}}\left(\mathbf{s}_{\eta_{i}}\right)\right)$. Since $\left|P_{\mathcal{R}}\left(\mathbf{s}_{\eta_{i}}\right)\right|_{\mathbf{L}^{2}} \leq \alpha$ by the definition of $\mathcal{R}$ and the projection map $P_{\mathcal{R}}$ is non-expansive, we have

$$
\left|\mathbf{s}_{\eta_{2}}-\mathbf{s}_{\eta_{1}}\right|_{q} \leq q^{1 / 2}\left|\eta_{2} P_{\mathcal{R}}\left(\mathbf{s}_{\eta_{2}}\right)-\eta_{1} P_{\mathcal{R}}\left(\mathbf{s}_{\eta_{1}}\right)\right|_{\mathbf{L}^{2}} \leq q^{1 / 2}\left(\eta_{2}\left|\mathbf{s}_{\eta_{2}}-\mathbf{s}_{\eta_{1}}\right|_{\mathbf{L}^{2}}+\alpha\left|\eta_{2}-\eta_{1}\right|\right) .
$$

From this it is straightforward to conclude

$$
\left|\mathbf{s}_{\eta_{2}}-\mathbf{s}_{\eta_{1}}\right|_{\mathbf{L}^{2}} \leq \frac{\alpha}{1-\eta_{2}}\left|\eta_{2}-\eta_{1}\right| \quad \text { and } \quad\left|\operatorname{div}\left(\mathbf{s}_{\eta_{2}}-\mathbf{s}_{\eta_{1}}\right)\right|_{L^{2}} \leq \frac{\alpha q^{1 / 2}}{1-\eta_{2}}\left|\eta_{2}-\eta_{1}\right| .
$$

Hence, $\eta \mapsto \mathbf{s}_{\eta}$ is locally Lipschitz continuous on $(0,1)$. This concludes the proof of $\mathbf{i}$.

We now proceed with the proof of ii. Take a sequence $\left\{\eta_{i}\right\}$ in $(0,1)$ such that $\eta_{i} \uparrow 1$ as $i \rightarrow \infty$ and consider the sequence $\left\{\mathbf{s}_{\eta_{i}}\right\}$ of fixed points $\mathbf{s}_{\eta_{i}}=T\left(\eta P_{\mathcal{R}}\left(\mathbf{s}_{\eta_{i}}\right)\right)=$ $\arg \min _{\mathbf{s} \in H_{0}(\mathrm{div})} \mathcal{G}_{n}^{\eta_{i}}(\mathbf{s})$. Then

$$
\begin{aligned}
\frac{1}{2}|f|_{L^{2}}^{2}=\mathcal{G}_{n}^{\eta_{i}}(0) & \geq \min _{\mathbf{s} \in H_{0}(\operatorname{div})} \mathcal{G}_{n}^{\eta_{i}}(\mathbf{s})=\mathcal{G}_{n}^{\eta_{i}}\left(\mathbf{s}_{\eta_{i}}\right) \\
& \geq \frac{1}{2}\left(\left|\operatorname{div} \mathbf{s}_{\eta_{\mathbf{i}}}\right|_{L^{2}}-|f|_{L^{2}}\right)^{2}+\frac{q}{2}\left(\left|\mathbf{s}_{\eta_{i}}\right|_{\mathbf{L}^{2}}-\eta_{i}\left|P_{\mathcal{R}}\left(\mathbf{s}_{\eta_{i}}\right)\right|_{\mathbf{L}^{2}}\right)^{2} .
\end{aligned}
$$

Since $\left|P_{\mathcal{R}}\left(\mathbf{s}_{\eta_{i}}\right)\right|_{\mathbf{L}^{2}} \leq \alpha$ for all $i$, the sequences $\left\{\left|\operatorname{divs}_{\eta_{\mathrm{i}}}\right|_{L^{2}}\right\}$ and $\left\{\left|\mathbf{s}_{\eta_{\mathrm{i}}}\right|_{\mathbf{L}^{2}}\right\}$ are uniformly bounded. Hence, $\left\{\mathbf{s}_{\eta_{i}}\right\}$ is bounded in $H_{0}(\operatorname{div})$, and since the latter is a reflexive space, there exists a subsequence of $\left\{\mathbf{s}_{\eta_{i}}\right\}$ (again denoted by $\left\{\mathbf{s}_{\eta_{i}}\right\}$ ) such that $\mathbf{s}_{\eta_{i}} \rightarrow \mathbf{s}^{*}$ for some $\mathbf{s}^{*} \in H_{0}(\operatorname{div})$ as $i \rightarrow \infty$.

We now prove that $\mathbf{s}^{*}$ is a minimizer of $\mathbf{s} \mapsto \mathcal{G}_{n}(\mathbf{s})$. For this purpose, we note that the sequence $\left\{P_{\mathcal{R}}\left(\mathbf{s}_{\eta_{i}}\right)\right\} \subset \mathcal{R}$ is bounded in $\mathbf{L}^{2}(\Omega)$. Hence, there exists 
a subsequence of $\left\{P_{\mathcal{R}}\left(\mathbf{s}_{\eta_{i}}\right)\right\}$ (also denoted by $\left\{P_{\mathcal{R}}\left(\mathbf{s}_{\eta_{i}}\right)\right\}$ ) for which $P_{\mathcal{R}}\left(\mathbf{s}_{\eta_{i}}\right) \rightarrow z$ for some $z \in \mathcal{R} \subset \mathbf{L}^{2}(\Omega)$. Since $\mathbf{s}_{\eta_{i}} \rightarrow \mathbf{s}^{*}$, we have $\left(\mathbf{s}_{\eta_{i}}-\eta_{i} P_{\mathcal{R}}\left(\mathbf{s}_{\eta_{i}}\right)\right) \rightarrow\left(\mathbf{s}^{*}-z\right)$ in $\mathbf{L}^{2}(\Omega)$ as $i \rightarrow \infty$. Here we use the fact that $H_{0}(\operatorname{div}) \hookrightarrow \mathbf{L}^{2}(\Omega)$ as well as $\mathbf{L}^{2}(\Omega)^{*} \equiv$ $\mathbf{L}^{2}(\Omega) \hookrightarrow H_{0}(\operatorname{div})^{*}$ are continuous, dense embeddings. Hence, if $\left\{z_{n}\right\} \subset H_{0}($ div $)$ such that $\left(l, z_{n}\right)_{H_{0}(\operatorname{div})^{*}, H_{0}(\operatorname{div})} \rightarrow(l, z)_{H_{0}(\operatorname{div})^{*}, H_{0}(\text { div })}$ for all $l \in H_{0}(\operatorname{div})^{*}$, then the same holds for $l \in \mathbf{L}^{2}(\Omega)$. Thus, since $z \in \mathcal{R}$, by the weak lower semicontinuity of the norm we have

$$
\left|\mathbf{s}^{*}-P_{\mathcal{R}}\left(\mathbf{s}^{*}\right)\right|_{\mathbf{L}^{2}} \leq\left|\mathbf{s}^{*}-z\right|_{\mathbf{L}^{2}} \leq \varliminf_{i \rightarrow \infty}\left|\mathbf{s}_{\eta_{i}}-\eta_{i} P_{\mathcal{R}}\left(\mathbf{s}_{\eta_{i}}\right)\right|_{\mathbf{L}^{2}},
$$

where we utilize the minimum distance property of the projection $P_{\mathcal{R}}$. Let $\mathbf{s} \in H_{0}(\operatorname{div})$ be arbitrary. Then

$$
\begin{aligned}
\frac{1}{2}\left|\operatorname{divs}^{*}+f\right|_{L^{2}}^{2}+\frac{q_{n}}{2} \mid \mathbf{s}^{*} & -\left.P_{\mathcal{R}}\left(\mathbf{s}^{*}\right)\right|_{\mathbf{L}^{2}} ^{2} \leq \frac{1}{2}\left|\operatorname{divs}^{*}+f\right|_{L^{2}}^{2}+\frac{q_{n}}{2}\left|\mathbf{s}^{*}-z\right|_{\mathbf{L}^{2}}^{2} \\
& \leq\left(\varliminf_{i \rightarrow \infty} \frac{1}{2}\left|\operatorname{div} \mathbf{s}_{\eta_{i}}+f\right|_{L^{2}}^{2}\right)+\left(\underline{\lim _{i \rightarrow \infty}} \frac{q_{n}}{2}\left|\mathbf{s}_{\eta_{i}}-\eta_{i} P_{\mathcal{R}}\left(\mathbf{s}_{\eta_{i}}\right)\right|_{\mathbf{L}^{2}}^{2}\right) \\
& \leq \varliminf_{i \rightarrow \infty}\left(\frac{1}{2}\left|\operatorname{divs} \mathbf{s}_{\eta_{i}}+f\right|_{L^{2}}^{2}+\frac{q_{n}}{2}\left|\mathbf{s}_{\eta_{i}}-\eta_{i} P_{\mathcal{R}}\left(\mathbf{s}_{\eta_{i}}\right)\right|_{\mathbf{L}^{2}}^{2}\right) \\
& =\varliminf_{i \rightarrow \infty} \mathcal{G}_{n}^{\eta_{i}}\left(\mathbf{s}_{\eta_{i}}\right) \leq \varliminf_{i \rightarrow \infty} \mathcal{G}_{n}^{\eta_{i}}(\mathbf{s}) \\
& =\varliminf_{i \rightarrow \infty}\left(\frac{1}{2}|\operatorname{div} \mathbf{s}+f|_{L^{2}}^{2}+\frac{q_{n}}{2}\left|\mathbf{s}-\eta_{i} P_{\mathcal{R}}(\mathbf{s})\right|_{\mathbf{L}^{2}}^{2}\right) \\
& =\frac{1}{2}|\operatorname{divs}+f|_{L^{2}}^{2}+\frac{q_{n}}{2}\left|\mathbf{s}-P_{\mathcal{R}}(\mathbf{s})\right|_{\mathbf{L}^{2}}^{2},
\end{aligned}
$$

i.e., $\mathcal{G}_{n}\left(\mathbf{s}^{*}\right)=\inf _{\mathbf{s} \in H_{0}(\operatorname{div})} \mathcal{G}_{n}(\mathbf{s})$.

Finally, we consider iii. Let $(\mathbf{s}, \mathbf{t}) \in H_{0}(\operatorname{div}) \times \mathbf{L}^{2}(\Omega)$ be arbitrary. Since we have proven that $\mathbf{s}^{*}$ is a minimizer of $\mathbf{s} \mapsto \mathcal{G}_{n}(\mathbf{s})$, from the minimum distance property of $P_{\mathcal{R}}$ we get

$$
\begin{aligned}
\mathcal{E}_{n}^{d}\left(\mathbf{s}^{*}, P_{\mathcal{R}}\left(\mathbf{s}^{*}\right)\right) & =\mathcal{G}_{n}\left(\mathbf{s}^{*}\right) \leq \mathcal{G}_{n}(\mathbf{s})=\frac{1}{2}|\operatorname{divs}+f|_{L^{2}}^{2}+\frac{q_{n}}{2}\left|\mathbf{s}-P_{\mathcal{R}}(\mathbf{s})\right|_{\mathbf{L}^{2}}^{2} \\
& \leq \frac{1}{2}|\operatorname{divs}+f|_{L^{2}}^{2}+\frac{q_{n}}{2}|\mathbf{s}-\mathbf{t}|_{\mathbf{L}^{2}}^{2}+\delta_{\mathcal{R}}(\mathbf{t}) \\
& =\mathcal{E}_{n}^{d}(\mathbf{s}, \mathbf{t}) .
\end{aligned}
$$

Henceforth, taking the infimum over $(\mathbf{s}, \mathbf{t}) \in H_{0}(\operatorname{div}) \times \mathbf{L}^{2}(\Omega)$ we obtain $\mathcal{E}_{n}^{d}\left(\mathbf{s}^{*}, P_{\mathcal{R}}\left(\mathbf{s}^{*}\right)\right) \leq \inf (\mathbf{s , t}) \in H_{0}(\operatorname{div}) \times \mathbf{L}^{2}(\Omega) \mathcal{E}_{n}^{d}(\mathbf{s}, \mathbf{t})$, i.e., $\left(\mathbf{s}^{*}, P_{\mathcal{R}}\left(\mathbf{s}^{*}\right)\right)$ is a minimizer of $\mathcal{E}_{n}^{d}$.

Remark on the role of $\eta \in(0,1)$. As we have proven in Theorem 3.1, the value of $\eta$ is the Lipschitz constant of the nonlinear operator that determines the sequence $\left\{\mathbf{s}_{\eta}^{k}\right\}$ via a fixed point iteration. Hence, based on the proof of the aforementioned theorem, $\eta \in(0,1)$ is required to obtain strong convergence in $H_{0}$ (div) of the sequence $\left\{\mathbf{s}_{\eta}^{k}\right\}$. Furthermore, numerical evidence of the necessity of $\eta \in(0,1)$ is provided in section 3.3.1.

One might be tempted to derive the contraction result of the proof in Theorem 3.1 by other more direct means using maximal monotone operator theory: In fact, Algorithm 2 can be written as an iteration of the type (FB) where $C=\eta, \lambda=1 / q$, $\mathbf{s} \mapsto A \mathbf{s}=(\operatorname{div})^{*}(\operatorname{divs}+f)$ and $B=q\left(I-P_{\mathcal{R}}\right)$ (see item (i) and (iv) in section 
3.1.3). Note, however, that the contractivity of $(I+\lambda A)^{-1} C(I-\lambda B)$ in $H_{0}$ (div) with $\eta \in(0,1)$ cannot be obtained simply from non-expansivity of $(I+\lambda A)^{-1}$ as $(I-\lambda B)$ is non-expansive only in $\mathbf{L}^{2}(\Omega)$.

3.1.3. The role of $\eta$ in Algorithm 2 and the limitation of classical splitting approaches applied to Algorithm 2. In this section we further substantiate the need of $\eta \in(0,1)$ in Algorithm 2 and of the results in Theorem 3.1. For this purpose we suppose that $\eta=1$ and analyse the scope of existing results for (FB) and (BB) (with $C=I$ ). In what follows we point to the shortcomings of these results for classical splitting algorithms when applied with $\mathscr{H}=H_{0}($ div $)$ or $\mathscr{H}=\mathbf{L}^{2}(\Omega)$.

Since our main interest is the behaviour of the sequence $\left\{\mathbf{s}^{k}\right\}_{k=1}^{\infty}$, we focus (without loss of generality) on the fixed point iteration in $\mathbf{s}^{k}$ generated by Algorithm 2 .

(i) (BB) with $\mathscr{H}=H_{0}\left(\right.$ div). Step 2 in Algorithm 2 can be written as $\left(I+\frac{1}{q} A\right) \mathbf{s}^{k}=$ $\mathbf{t}^{k-1}$ with $H_{0}(\operatorname{div}) \ni \mathbf{s} \mapsto A \mathbf{s}=(\operatorname{div})^{*}(\operatorname{div} \mathbf{s}+f) \in H_{0}(\operatorname{div})$ (via the Riesz map) and step 3 is simply given by $\mathbf{t}^{k}=P_{\mathcal{R}}\left(\mathbf{s}^{k}\right)$ where $P_{\mathcal{R}}$ is the $\mathbf{L}^{2}(\Omega)$-projection operator onto the closed convex set $\mathcal{R}$. It is known that for any $\lambda>0$ one has $P_{\mathcal{R}}=\left(I+\lambda \partial \delta_{\mathcal{R}}\right)^{-1}$ where $\partial \delta_{\mathcal{R}}$ is the subdifferential (in $\mathbf{L}^{2}(\Omega)$ ) of the indicator function $\delta_{\mathcal{R}}$ of the set $\mathcal{R}$. It is well known that $\partial \delta_{\mathcal{R}}$ is maximal monotone (see, e.g., Rockafellar's theorem [34]). Then, in particular for $\lambda=\frac{1}{q}$, we have that $\mathbf{s}^{k}=\left(I+\frac{1}{q} A\right)^{-1}\left(I+\frac{1}{q} B\right)^{-1} \mathbf{s}^{k-1}$ with $B=\partial \delta_{\mathcal{R}}$. Structurally, this iteration is of the form (BB). However, $\partial \delta_{\mathcal{R}}$ is in general not maximal monotone on $H_{0}$ (div). Additionally, $B$ is neither Lipschitz continuous nor strongly monotone over $\mathscr{H}$ and neither $A$ nor $B$ are coercive. Hence, as mentioned in the paragraph following the definition of $(\mathrm{BB})$ and regarding on classical convergence results for splitting methods, no guarantees concerning the strong convergence of the sequence of iterates generated by (BB) can be given. Furthermore, in case a subsequence of iterates is weakly convergent, it does not converge to a zero of $A+B$ (see $[12,29,30])$. Our approach, however, guarantees strong convergence of $\mathbf{s}_{\eta}^{k}$ to $\mathbf{s}_{\eta}^{*}$ in $H_{0}$ (div) as $k \rightarrow \infty$ and weak convergence of $\mathbf{s}_{\eta_{i}}^{*}$ in $H_{0}($ div $)$ to $\mathbf{s}^{*}$ such that the pair $\left(\mathbf{s}^{*}, P_{\mathcal{R}}\left(\mathbf{s}^{*}\right)\right) \in H_{0}(\operatorname{div}) \times \mathbf{L}^{2}(\Omega)$ is a solution to $(20)$.

As a final comment, consider the following: The set $\tilde{\mathcal{R}}:=\mathcal{R} \cap H_{0}$ (div) is closed, convex and non-empty in $H_{0}(\mathrm{div})$. The minimization problem in step 3 could be considered in $H_{0}$ (div) (with $\mathcal{R}$ exchanged by $\tilde{\mathcal{R}}$ ). Then, this step is equivalent to $\mathbf{t}^{k}=\tilde{P}_{\tilde{\mathcal{R}}}\left(\mathbf{s}^{k}\right)$. However, $\tilde{P}_{\tilde{\mathcal{R}}}$ is now the projection in $H_{0}(\operatorname{div})$, which is unfortunately more complicated to compute than the projection in $\mathbf{L}^{2}(\Omega)$. Indeed, the latter can be obtained explicitly as

$$
P_{\mathcal{R}}(\mathbf{s})(\mathbf{x})= \begin{cases}\mathbf{s}(\mathbf{x}) & |\mathbf{s}(\mathbf{x})| \leq \alpha \\ \frac{\mathbf{s}(\mathbf{x})}{|\mathbf{s}(\mathbf{x})|} & \text { otherwise }\end{cases}
$$

The projection $\tilde{P}_{\tilde{\mathcal{R}}}(\mathbf{s})$, on the other hand, requires to solve

$$
\min \frac{1}{2}|\operatorname{divs}-\operatorname{div} \mathbf{t}|_{L^{2}}^{2}+\frac{1}{2}|\mathbf{s}-\mathbf{t}|_{\mathbf{L}^{2}}^{2}, \quad \text { over } \mathbf{t} \in \tilde{\mathcal{R}}
$$

(ii) (BB) with $\mathscr{H}=\mathbf{L}^{2}(\Omega)$. The map $\mathbf{s} \mapsto F(\mathbf{s}):=\frac{1}{2} \mid$ divs $+\left.f\right|_{L^{2}} ^{2}$ is convex, proper and lower semi-continuous on $\mathbf{L}^{2}(\Omega)$ with effective domain $\operatorname{dom}(F)=H(\operatorname{div}):=$ 
$\left\{\mathbf{v} \in \mathbf{L}^{2}(\Omega): \operatorname{divs} \in L^{2}(\Omega)\right\}$. Its subdifferential $\partial F$ is maximal monotone and step 2 can, thus, be written as $\mathbf{s}^{k}=\left(I+\frac{1}{q} A\right)^{-1} \mathbf{t}^{k-1}$ with $A=\partial F$. As in the previous paragraph, $\mathbf{t}^{k}=P_{\mathcal{R}} \mathbf{s}^{k}=\left(I+\frac{1}{q} B\right)^{-1} \mathbf{s}^{k}$ with $B=\partial \delta_{\mathcal{R}}$. Hence, Algorithm 2 is equivalent to the (BB) iteration with $\lambda=1 / q$. As in the previous paragraph, note that $B$ is neither Lipschitz continuous nor strongly monotone over $\mathscr{H}$ and neither $A$ nor $B$ are coercive. As a consequence, strong convergence of the iteration cannot be obtained by standard theoretic arguments, to the best of our knowledge. Again as before, weak cluster points of the iteration are not related to the original problem.

(iii) (FB) with $\mathscr{H}=H_{0}$ (div). In order to pose the problem as an (FB)-iteration, it is necessary to consider $(I-\lambda B)=P_{\mathcal{R}}$, where $P_{\mathcal{R}}$ is the $\mathbf{L}^{2}(\Omega)$-projector onto the closed convex set $\mathcal{R}$. But now, it follows that $B$ is no longer maximal monotone over $\mathscr{H}$. Hence, standard theory for splitting methods does not apply to this setting .

(iv) (FB) with $\mathscr{H}=\mathbf{L}^{2}(\Omega)$. From the previous paragraph, we observe that $B=\left(I-P_{\mathcal{R}}\right) / \lambda$ and since $P_{\mathcal{R}}=\left(I+\lambda \partial \delta_{\mathcal{R}}\right)^{-1}, B$ is the Fréchet derivative of the Moreau-Yosida regularization $\varphi_{\lambda}(\cdot)$ of $\delta_{\mathcal{R}}$, which is given by $\varphi_{\lambda}(\mathbf{s}):=$ $\min _{\mathbf{t} \in \mathbf{L}^{2}(\Omega)} \frac{1}{2 \lambda}|\mathbf{s}-\mathbf{t}|_{\mathbf{L}^{2}}^{2}+\delta_{\mathcal{R}}(\mathbf{t})$ (see [37]). Since $\varphi_{\lambda}$ is convex, we can write the iteration in the form of (FB), with $\lambda=1 / q, B=\varphi_{1 / q}^{\prime}$ and $A=\partial F$ (where $F$ is as in item ii). Although in some cases it can be proven that the generated sequence converges weakly to a zero of $A+B$ (see for example [20]), it is not clear that this statement is true in general. In fact, general results that guarantee weak convergence require the update of $\lambda$ in each step leading to a sequence $\left\{\lambda_{k}\right\} \in \ell^{2} \backslash \ell^{1}$. Then, convergence is restricted to ergodic means of the iteration (see [33]). Note also that Bruck's paper [5], which is cited in [30] related to weak convergence of this iteration, has been corrected in [6] where the conditions necessary for proving convergence have been strengthened considerably.

Furthermore, to the best of our knowledge, known sufficient conditions for the strong convergence of this iteration involve the strong monotonicity of at least one of the operators $A$ and $B$ (see Chapter 4 in [12] for a list of these conditions) or the strong monotonicity of the operator $B^{-1}$. Such conditions, however, do not hold true in our case.

(v) All the aforementioned issues persist if one now considers the fixed-point iteration in $\mathbf{t}^{k}$ (rather than in $\mathbf{s}^{k}$ ) generated in Algorithm 2 and performs the analogous analysis as in items (i) - (iv).

Above we have comprehensively argued that the existing function space results in the literature concerning convergence of splitting methods of the form (FB) and (BB) (with $C=I$, i.e., $\eta=1$ ) are even in the best case scenario not suitable to yield convergence properties of Algorithm 2. In contrast, Theorem 3.1, yields strong convergence of the aforementioned algorithm in $H_{0}(\mathrm{div})$. It should also be noted that the requirement of $\eta \in(0,1)$ cannot be relaxed to $\eta \in(0,1]$ as our numerical evidence in Figure 1 demonstrates.

We further emphasize the significance of $H_{0}$ (div)-convergence. In fact, according to (17), given a sequence $\mathbf{s}^{k}$ converging to $\overline{\mathbf{s}}$ in $H_{0}$ (div) the corresponding sequence of images is given by

$$
u^{k}=\operatorname{divs}^{k}+f
$$


The $H_{0}$ (div)-convergence of $\mathbf{s}^{k}$ yields $L^{2}$-convergence of divs ${ }^{k}$ to div $\overline{\mathbf{s}}$ and thus $u^{k} \rightarrow \bar{u}$ in $L^{2}(\Omega)$ as $k \rightarrow \infty$. Moreover, when $\overline{\mathbf{s}}$ solves the Fenchel pre-dual problem (16), then Fenchel duality arguments yield $\bar{u} \in \mathrm{BV}(\Omega)$.

\subsection{Augmented Lagrangian method for the pre-dual problem}

In order to avoid a limiting process of the penalty parameter $q_{n}$ in (20), we prove that an approach based on the augmented Lagrangian method is suitable for the problem in this respect. For this purpose, let $Y:=H_{0}(\operatorname{div}) \times H_{0}(\operatorname{div})$ and consider the following family of problems with regularization parameter $\epsilon \geq 0$ :

$$
\begin{aligned}
& \min \mathcal{J}_{d}^{\epsilon}(\mathbf{s}):=\frac{1}{2}|\operatorname{divs}+f|_{L^{2}}^{2}+\frac{\epsilon}{2}|\mathbf{s}|_{\mathbf{L}^{2}}^{2} \quad \text { over }(\mathbf{s}, \mathbf{t}) \in Y, \\
& \text { s.t. } \quad \mathbf{t} \in \tilde{\mathcal{R}} \text { and } g(\mathbf{s}, \mathbf{t}):=\mathbf{s}-\mathbf{t}=0,
\end{aligned}
$$

with $\mathcal{J}_{d}^{\epsilon}: H_{0}$ (div) $\rightarrow \mathbb{R}, g: Y \rightarrow H_{0}$ (div) and $\tilde{\mathcal{R}}=\mathcal{R} \cap H_{0}$ (div). We note that the the $\epsilon$-term is for the sake of generality only, with the advantage of the existence of a unique solution to (28) for $\epsilon>0$. For the choice $\epsilon=0$, on the other hand, (28) is equivalent to the pre-dual problem (16).

Concerning existence of a solution, we note that the objective $\mathcal{J}_{d}^{\epsilon}$ is coercive, convex (strictly convex if $\epsilon>0$ ) and continuous (and hence lower semicontinuous) and $\tilde{\mathcal{R}}$ is closed, convex and non-empty. Therefore there exists a solution $\mathbf{s}^{*}$ which is unique if $\epsilon>0$ that solves the minimization problem (28) and satisfies $\mathbf{s}^{*} \in \tilde{\mathcal{R}}$.

Next, we consider the Lagrangian $\mathcal{L}^{d}: Y \times H_{0}(\mathrm{div})^{*} \rightarrow \mathbb{R} \cup\{+\infty\}$ associated with (28) and the pertinent augmented Lagrangian $\mathcal{L}_{\text {Aug }}^{d}: Y \times H_{0}(\operatorname{div})^{*} \times \mathbb{R}^{+} \rightarrow \mathbb{R} \cup\{+\infty\}$ defined as

$$
\begin{aligned}
\mathcal{L}^{d}(\mathbf{s}, \mathbf{t}, \boldsymbol{\lambda}) & :=\frac{1}{2}|\operatorname{divs}+f|_{L^{2}}^{2}+\frac{\epsilon}{2}|\mathbf{s}|_{\mathbf{L}^{2}}^{2}+\delta_{\tilde{\mathcal{R}}}(\mathbf{t})+\langle\boldsymbol{\lambda}, \mathbf{s}-\mathbf{t}\rangle, \\
\mathcal{L}_{\text {Aug }}^{d}(\mathbf{s}, \mathbf{t}, \boldsymbol{\lambda}, q) & :=\frac{1}{2}|\operatorname{divs}+f|_{L^{2}}^{2}+\frac{\epsilon}{2}|\mathbf{s}|_{\mathbf{L}^{2}}^{2}+\delta_{\tilde{\mathcal{R}}}(\mathbf{t})+\langle\boldsymbol{\lambda}, \mathbf{s}-\mathbf{t}\rangle+\frac{q}{2}|\mathbf{s}-\mathbf{t}|_{H_{0}(\text { div })}^{2},
\end{aligned}
$$

for $q>0$. Here and below, " $\langle\cdot, \cdot\rangle$ " denotes the duality pairing between $H_{0}(\operatorname{div})^{*}$ and $H_{0}(\operatorname{div})$.

Since the mapping $g$ in $(28 \mathrm{~b})$ is Fréchet differentiable and $D g(\mathbf{s}, \mathbf{t}) \in$ $\mathscr{L}\left(Y, H_{0}(\right.$ div $\left.)\right)$, given by $D g(\mathbf{s}, \mathbf{t})\left(\mathbf{h}_{1}, \mathbf{h}_{2}\right)=\mathbf{h}_{1}-\mathbf{h}_{2}$, we observe that for any $(\mathbf{s}, \mathbf{t})$

$$
\begin{aligned}
0 \in \operatorname{int}\left\{D g(\mathbf{s}, \mathbf{t})\left(\left(\mathbf{r}_{1}, \mathbf{r}_{2}\right)-(\mathbf{s}, \mathbf{t})\right)+g(\mathbf{s}, \mathbf{t}): \mathbf{r}_{1} \in H_{0}(\operatorname{div}), \mathbf{r}_{2} \in \tilde{\mathcal{R}}\right\} \equiv \\
\quad \operatorname{int}\left\{\mathbf{r}_{1}-\mathbf{r}_{2}: \mathbf{r}_{1} \in H_{0}(\operatorname{div}), \mathbf{r}_{2} \in \tilde{\mathcal{R}}\right\},
\end{aligned}
$$

where the interior is taken in $H_{0}$ (div). As a consequence, by standard surjectivity techniques (see $[27,44]$ ) there exists a Lagrange multiplier $\boldsymbol{\lambda}^{*} \in H_{0}(\operatorname{div})^{*}$ such that $\left\langle\boldsymbol{\lambda}^{*}, \mathbf{s}^{*}-\mathbf{t}^{*}\right\rangle=0$ and

$$
D \mathcal{J}_{d}^{\epsilon}\left(\mathbf{s}^{*}\right)+\boldsymbol{\lambda}^{*} \circ D g\left(\mathbf{s}^{*}, \mathbf{t}^{*}\right) \in-\mathcal{R}\left(\mathbf{s}^{*}, \mathbf{t}^{*}\right)^{+},
$$

where $\mathcal{R}\left(\mathbf{s}^{*}, \mathbf{t}^{*}\right)^{+}$is the polar cone of the conical hull of $\mathcal{R} \backslash\left(\mathbf{s}^{*}, \mathbf{t}^{*}\right)$, i.e.,

$-\mathcal{R}\left(\mathbf{s}^{*}, \mathbf{t}^{*}\right)^{+}=\left\{F \in Y^{*}:\left(F,\left(\mathbf{s}^{*}, \mathbf{t}^{*}\right)\right)_{Y^{*}, Y} \leq\left(F,\left(\mathbf{r}_{1}, \mathbf{r}_{2}\right)\right)_{Y^{*}, Y}, \forall \mathbf{r}_{1} \in H_{0}(\operatorname{div}), \mathbf{r}_{2} \in \tilde{\mathcal{R}}\right\}$.

Hence (31) is equivalent to

$$
\begin{aligned}
& \left(\operatorname{divs}^{*}+f, \operatorname{divs}^{*}\right)_{L^{2}}+\epsilon\left(\mathbf{s}^{*}, \mathbf{s}^{*}\right)_{\mathbf{L}^{2}}+\left\langle\boldsymbol{\lambda}^{*}, \mathbf{s}^{*}-\mathbf{t}^{*}\right\rangle \leq \\
& \left(\operatorname{divs}^{*}+f, \operatorname{div} \mathbf{r}_{1}\right)_{L^{2}}+\epsilon\left(\mathbf{s}^{*}, \mathbf{r}_{1}\right)_{\mathbf{L}^{2}}+\left\langle\boldsymbol{\lambda}^{*}, \mathbf{r}_{1}-\mathbf{r}_{2}\right\rangle, \quad \forall \mathbf{r}_{1} \in H_{0}(\operatorname{div}), \mathbf{r}_{2} \in \tilde{\mathcal{R}} .
\end{aligned}
$$


Here we use $D \mathcal{J}_{d}^{\epsilon}(\mathbf{s})\left(\mathbf{h}_{1}, \mathbf{h}_{2}\right)=\left(\operatorname{divs}+f, \operatorname{divh} \mathbf{h}_{1}\right)_{L^{2}}+\epsilon\left(\mathbf{s}, \mathbf{h}_{1}\right)_{\mathbf{L}^{2}}$ for all $(\mathbf{s}, \mathbf{t}),\left(\mathbf{h}_{1}, \mathbf{h}_{2}\right) \epsilon$ $Y$. This implies that

$$
\left\langle\boldsymbol{\lambda}^{*}, \mathbf{s}^{*}-\mathbf{t}^{*}\right\rangle \leq\left\langle\boldsymbol{\lambda}^{*}, \mathbf{r}_{1}-\mathbf{r}_{2}\right\rangle+D \mathcal{J}_{d}^{\epsilon}\left(\mathbf{s}^{*}\right)\left(\mathbf{r}_{1}-\mathbf{s}^{*}\right) \quad \forall \mathbf{r}_{1} \in H_{0}(\operatorname{div}), \mathbf{r}_{2} \in \tilde{\mathcal{R}} .
$$

Theorem 3.2 Let $\left(\mathbf{s}^{*}, \mathbf{t}^{*}\right) \in Y$ be a solution ( which is unique for $\epsilon>0$ ) to (28), and let $\boldsymbol{\lambda}^{*}$ be the associated Lagrange multiplier. Then, for $q>0,\left(\mathbf{s}^{*}, \mathbf{t}^{*}\right)$ is a global minimizer (unique for $\epsilon>0$ ) of the map

$$
Y \ni(\mathbf{s}, \mathbf{t}) \mapsto \mathcal{L}_{\text {Aug }}^{d}\left(\mathbf{s}, \mathbf{t}, \lambda^{*}, q\right) .
$$

Proof. Let $\mathbf{s} \in H_{0}(\mathrm{div})$. Then, it is straightforward to observe that $D^{2} \mathcal{J}_{d}^{\epsilon}(\mathbf{s}) \in$ $\mathscr{L}\left(H_{0}(\operatorname{div}) \times H_{0}(\operatorname{div}), \mathbb{R}\right)$ is given by

$$
D^{2} \mathcal{J}_{d}^{\epsilon}(\mathbf{s})\left(\mathbf{h}_{1}, \mathbf{h}_{2}\right)=\left(\operatorname{div} \mathbf{h}_{2}, \operatorname{divh} \mathbf{h}_{1}\right)_{L^{2}}+\epsilon\left(\mathbf{h}_{2}, \mathbf{h}_{1}\right)_{\mathbf{L}^{2}}, \quad \forall \mathbf{h}_{1}, \mathbf{h}_{2} \in H_{0}(\operatorname{div}) .
$$

Consequently, $D^{3} \mathcal{J}_{d}^{\epsilon}(\mathbf{s})=0$. Assuming that $0<\epsilon<1$, we have $D^{2} \mathcal{J}_{d}^{\epsilon}(\mathbf{s})(\mathbf{h}, \mathbf{h})=$ $|\operatorname{divh}|_{L^{2}}^{2}+\epsilon|\mathbf{h}|_{\mathbf{L}^{2}}^{2} \geq \epsilon|\mathbf{h}|_{H_{0}(\text { div) }}^{2}$. We also have the following representation based on a Taylor series expansion:

$$
\mathcal{J}_{d}^{\epsilon}(\mathbf{s}+\mathbf{h})=\mathcal{J}_{d}^{\epsilon}(\mathbf{s})+D \mathcal{J}_{d}^{\epsilon}(\mathbf{s})(\mathbf{h})+\frac{1}{2} D^{2} \mathcal{J}_{d}^{\epsilon}(\mathbf{s})(\mathbf{h}, \mathbf{h}), \quad \forall \mathbf{h} \in H_{0}(\operatorname{div}) .
$$

Since $\mathcal{L}_{\text {Aug }}^{d}\left(\mathbf{s}, \mathbf{t}, \boldsymbol{\lambda}^{*}, q\right)=+\infty$ if $\mathbf{t} \notin \tilde{\mathcal{R}}$, it is enough to consider $(\mathbf{s}, \mathbf{t}) \in H_{0}(\operatorname{div}) \times \tilde{\mathcal{R}}$. Since $\left(\mathbf{s}^{*}, \mathbf{t}^{*}\right) \in Y$ is a solution to $(28)$, we have that $\left|\mathbf{s}^{*}-\mathbf{t}^{*}\right|_{H_{0}(\text { div })}=0$. Then, by the previous paragraph and (32), we have the following chain of inequalities:

$$
\begin{aligned}
\mathcal{L}_{\text {Aug }}^{d}\left(\mathbf{s}^{*}, \mathbf{t}^{*}, \boldsymbol{\lambda}^{*}, q\right) & =\mathcal{J}_{d}^{\epsilon}\left(\mathbf{s}^{*}\right)+\delta_{\tilde{\mathcal{R}}}\left(\mathbf{t}^{*}\right)+\left\langle\boldsymbol{\lambda}^{*}, \mathbf{s}^{*}-\mathbf{t}^{*}\right\rangle \\
& \leq \mathcal{J}_{d}^{\epsilon}\left(\mathbf{s}^{*}\right)+\delta_{\tilde{\mathcal{R}}}\left(\mathbf{t}^{*}\right)+\left\langle\boldsymbol{\lambda}^{*}, \mathbf{s}-\mathbf{t}\right\rangle+D \mathcal{J}_{d}^{\epsilon}\left(\mathbf{s}^{*}\right)\left(\mathbf{s}-\mathbf{s}^{*}\right) \\
& =\mathcal{J}_{d}^{\epsilon}\left(\mathbf{s}^{*}\right)+\delta_{\tilde{\mathcal{R}}}(\mathbf{t})+\left\langle\boldsymbol{\lambda}^{*}, \mathbf{s}-\mathbf{t}\right\rangle+D \mathcal{J}_{d}^{\epsilon}\left(\mathbf{s}^{*}\right)\left(\mathbf{s}-\mathbf{s}^{*}\right) \\
& =\mathcal{J}_{d}^{\epsilon}(\mathbf{s})-\frac{1}{2} D^{2} \mathcal{J}_{d}^{\epsilon}\left(\mathbf{s}^{*}\right)\left(\mathbf{s}-\mathbf{s}^{*}, \mathbf{s}-\mathbf{s}^{*}\right)+\delta_{\tilde{\mathcal{R}}}(\mathbf{t})+\left\langle\boldsymbol{\lambda}^{*}, \mathbf{s}-\mathbf{t}\right\rangle \\
& =\mathcal{L}_{\text {Aug }}^{d}\left(\mathbf{s}, \mathbf{t}, \boldsymbol{\lambda}^{*}, q\right)-\frac{1}{2} D^{2} \mathcal{J}_{d}^{\epsilon}\left(\mathbf{s}^{*}\right)\left(\mathbf{s}-\mathbf{s}^{*}, \mathbf{s}-\mathbf{s}^{*}\right)-\frac{q}{2}|\mathbf{s}-\mathbf{t}|_{H_{0}(\text { div })}^{2} .
\end{aligned}
$$

For $\epsilon>0$, we obtain

$$
\mathcal{L}_{\text {Aug }}^{d}\left(\mathbf{s}^{*}, \mathbf{t}^{*}, \boldsymbol{\lambda}^{*}, q\right) \leq \mathcal{L}_{\text {Aug }}^{d}\left(\mathbf{s}, \mathbf{t}, \boldsymbol{\lambda}^{*}, q\right)-\frac{\epsilon}{2}\left|\mathbf{s}-\mathbf{s}^{*}\right|_{H_{0}(\text { div })}^{2}-\frac{q}{2}|\mathbf{s}-\mathbf{t}|_{H_{0}(\text { div })}^{2},
$$

and for $\epsilon=0$, we find

$$
\mathcal{L}_{\text {Aug }}^{d}\left(\mathbf{s}^{*}, \mathbf{t}^{*}, \boldsymbol{\lambda}^{*}, q\right) \leq \mathcal{L}_{\text {Aug }}^{d}\left(\mathbf{s}, \mathbf{t}, \boldsymbol{\lambda}^{*}, q\right)-\frac{1}{2}\left|\operatorname{div}\left(\mathbf{s}-\mathbf{s}^{*}\right)\right|_{L^{2}}^{2}-\frac{q}{2}|\mathbf{s}-\mathbf{t}|_{H_{0}(\operatorname{div})}^{2} .
$$

Therefore, $\mathcal{L}_{\text {Aug }}^{d}\left(\mathbf{s}^{*}, \mathbf{t}^{*}, \boldsymbol{\lambda}^{*}, q\right) \leq \mathcal{L}_{\text {Aug }}^{d}\left(\mathbf{s}, \mathbf{t}, \boldsymbol{\lambda}^{*}, q\right)$ for any $(\mathbf{s}, \mathbf{t}) \in Y$, i.e., $\left(\mathbf{s}^{*}, \mathbf{t}^{*}\right)$ is a global minimizer.

For $\epsilon>0$, if the equality $\mathcal{L}_{\text {Aug }}^{d}\left(\mathbf{s}^{*}, \mathbf{t}^{*}, \boldsymbol{\lambda}^{*}, q\right)=\mathcal{L}_{\text {Aug }}^{d}\left(\mathbf{s}, \mathbf{t}, \boldsymbol{\lambda}^{*}, q\right)$ holds true, then $\mathbf{s}=\mathbf{s}^{*}=\mathbf{t}$. Additionally, since $\left(\mathbf{s}^{*}, \mathbf{t}^{*}\right)$ is the unique solution to $(28)$, we have that 
$g\left(\mathbf{s}^{*}, \mathbf{t}^{*}\right)=\mathbf{s}^{*}-\mathbf{t}^{*}=0$ by $(28 \mathrm{~b})$. Hence, $(\mathbf{s}, \mathbf{t})=\left(\mathbf{s}^{*}, \mathbf{t}^{*}\right)$ and the uniqueness of the global minimizer follows.

Remark. If $\frac{q}{2}|\mathbf{s}-\mathbf{t}|_{H_{0}(\text { div) }}^{2}$ in the definition of $\mathcal{L}_{\text {Aug }}^{d}$ is replaced by $\frac{q}{2}|\mathbf{s}-\mathbf{t}|_{\mathbf{L}^{2}(\Omega)}^{2}$, then the assertion of Theorem 3.2 still hold true. However, as we see in what follows, such a change restricts the scope of the resulting augmented-Lagrangian-type method.

The augmented Lagrangian method for solving problem (28) operates as follows: Given some $\boldsymbol{\lambda}^{0} \in H_{0}(\operatorname{div})^{*}$, iterate for $k=1,2, \ldots$ :

$$
\begin{aligned}
& \text { compute }\left(\mathbf{s}^{k}, \mathbf{t}^{k}\right) \in \underset{(\mathbf{s}, \mathbf{t}) \in H_{0}(\operatorname{div}) \times \tilde{\mathcal{R}}}{\arg \min } \mathcal{L}_{c}^{d}\left(\mathbf{s}, \mathbf{t}, \boldsymbol{\lambda}^{k-1}, q\right), \\
& \text { update } \boldsymbol{\lambda}^{k}=\boldsymbol{\lambda}^{k-1}+q\left(\mathbf{s}^{k}-\mathbf{t}^{k}\right),
\end{aligned}
$$

with

$$
\mathcal{L}_{c}^{d}(\mathbf{s}, \mathbf{t}, \boldsymbol{\lambda}, q):=\frac{1}{2}|\operatorname{divs}+f|_{L^{2}}^{2}+\frac{\epsilon}{2}|\mathbf{s}|_{\mathbf{L}^{2}}^{2}+\langle\boldsymbol{\lambda}, \mathbf{s}-\mathbf{t}\rangle+\frac{q}{2}|\mathbf{s}-\mathbf{t}|_{H_{0}(\operatorname{div})}^{2} .
$$

Although the study of the convergence of (33) is beyond the scope of the present paper (in particular the convergence of inexact variants like the one highlighted in the discrete setting in Algorithm 4 below), several subtleties of the algorithm owing to the function space context should be pointed out. If $\boldsymbol{\lambda}^{0} \in H_{0}$ (div) $\subset H_{0}(\operatorname{div})^{*}$, then $\boldsymbol{\lambda}^{k} \in H_{0}$ (div) for all $k \in \mathbb{N}$ given the fact that $\left(\mathbf{s}^{k}, \mathbf{t}^{k}\right) \in Y$. In this case, the resolution of (33a) is similar to the one of (20) and a splitting scheme like the one of Algorithm 2 can be utilized. In fact, provided $\eta \in(0,1)$, for every $k \in \mathbb{N}$ we may consider

$$
\begin{aligned}
& \mathbf{s}_{\eta}^{k, j+1}=\underset{\mathbf{s} \in H_{0}(\text { div })}{\arg \min } \frac{1}{2}|\operatorname{divs}+f|_{L^{2}}^{2}+\frac{\epsilon}{2}|\mathbf{s}|_{\mathbf{L}^{2}}^{2}+\left\langle\boldsymbol{\lambda}^{k-1}, \mathbf{s}\right\rangle+\frac{q}{2}\left|\mathbf{s}-\eta \mathbf{t}_{\eta}^{k, j}\right|_{H_{0}(\text { div })}^{2}, \\
& \mathbf{t}_{\eta}^{k, j+1}=\underset{\mathbf{t} \in H_{0}(\text { div })}{\arg \min } \frac{q}{2}\left|\mathbf{s}_{\eta}^{k, j}-\mathbf{t}\right|_{H_{0}(\text { div })}^{2}-\left\langle\boldsymbol{\lambda}^{k-1}, \mathbf{t}\right\rangle+\delta_{\tilde{\mathcal{R}}}(\mathbf{t}) .
\end{aligned}
$$

A slight modification of the arguments in the proof of Theorem 3.1 yields that $\left(\mathbf{s}_{\eta}^{k, j}, \mathbf{t}_{\eta}^{k, j}\right) \rightarrow\left(\mathbf{s}_{\eta}^{k}, \mathbf{t}_{\eta}^{k}\right)$ as $j \rightarrow \infty$ and $\left(\mathbf{s}_{\eta}^{k}, \mathbf{t}_{\eta}^{k}\right) \rightarrow\left(\mathbf{s}^{k}, \mathbf{t}^{k}\right)$ as $\eta \uparrow 1$ in $Y$, respectively. In the general case where $\boldsymbol{\lambda}^{0} \in H_{0}(\operatorname{div})^{*}$ but $\boldsymbol{\lambda}^{0} \notin H_{0}$ (div), the resolution of (34) requires the inverse of the Riesz map in order to replace $\boldsymbol{\lambda}^{k-1}$ by its $H_{0}$ (div)-representation.

If $\frac{q}{2}|\mathbf{s}-\mathbf{t}|_{H_{0}(\text { div) }}^{2}$ in the definition of $\mathcal{L}_{c}^{d}$ is replaced by $\frac{q}{2}|\mathbf{s}-\mathbf{t}|_{\mathbf{L}^{2}(\Omega)}^{2}$, then (33a) is defined over $H_{0}($ div $) \times \mathcal{R}$, provided that $\boldsymbol{\lambda}^{k-1} \in \mathbf{L}^{2}(\Omega)$ and consequently $\boldsymbol{\lambda}^{0} \in \mathbf{L}^{2}(\Omega)$, as well. Hence, if $\boldsymbol{\lambda}^{0} \in \mathbf{L}^{2}(\Omega)$, then $\boldsymbol{\lambda}^{k} \in \mathbf{L}^{2}(\Omega)$ for all $k \in \mathbb{N}$. In this case, in order to approximate (33a) we may consider the analogue of (34) with the obvious changes: $H_{0}$ (div)-norms are replaced by $\mathbf{L}^{2}(\Omega)$-norms, $\tilde{\mathcal{R}}$ is replaced by $\mathcal{R}$ and problem $(34 \mathrm{~b})$ is posed in $\mathbf{L}^{2}(\Omega)$. Finally, a similar argument as above yields $\left(\mathbf{s}_{\eta}^{k, j}, \mathbf{t}_{\eta}^{k, j}\right) \rightarrow\left(\mathbf{s}_{\eta}^{k}, \mathbf{t}_{\eta}^{k}\right)$ as $j \rightarrow \infty$ and $\left(\mathbf{s}_{\eta}^{k}, \mathbf{t}_{\eta}^{k}\right) \rightarrow\left(\mathbf{s}^{k}, \mathbf{t}^{k}\right)$ as $\eta \uparrow 1$ in $H_{0}(\operatorname{div}) \times \mathbf{L}^{2}(\Omega)$, respectively. In general, however, one should not expect that $\left\{\boldsymbol{\lambda}^{k}\right\}$ is bounded in $\mathbf{L}^{2}(\Omega)$, as only $\boldsymbol{\lambda}^{*} \in H_{0}(\operatorname{div})$ is guaranteed.

It should further be noticed that upon discretization (and whenever mesh independence properties are not indispensable), the term $|\mathbf{s}-\mathbf{t}|_{H_{0}(\mathrm{div})}^{2}$ in $\mathcal{L}_{c}^{d}$ and consequently in (34) may be replaced by the squared discrete $\mathbf{L}^{2}(\Omega)$-norm of the difference of the discrete counterparts of $\mathbf{s}$ and $\mathbf{t}$. This simplifies the realization of (34b) considerably (even in function space), but the overall scheme does not admit a function space counterpart with convergence guarantees, in general. Such a finite dimensional approach with inexact subproblem solutions is considered in section 3.3.2 below. 


\subsection{Discrete pre-dual approaches}

In this section we study the methods of the previous solvers for the pre-dual problem (pre-dual splitting and augmented Lagrangian methods) in a discrete setting.

3.3.1. Discrete pre-dual splitting. For a given $N \times N$ image $f \in \mathbb{R}^{N^{2}}$, we denote by $\Omega_{h}=\{1, \ldots, N\} \times\{1, \ldots, N\}$ the discretization of $\Omega=(0,1) \times(0,1)$ with mesh size $h=\frac{1}{N-1}$. The necessary modifications in case of $\Omega_{h}=\{1, \ldots, M\} \times\{1, \ldots, N\}$ with $M \neq N$ are obvious. The discrete optimization problem associated to (20) is as follows:

$$
\begin{aligned}
& \min \mathcal{E}_{n, h}^{d}(\mathbf{s}, \mathbf{t}) \quad \text { over }(\mathbf{s}, \mathbf{t}) \in Y_{h}=\mathbb{R}^{2 N^{2}} \times \mathbb{R}^{2 N^{2}}, \\
& \mathcal{E}_{n, h}^{d}(\mathbf{s}, \mathbf{t}):=h^{2} \sum_{(i, j) \in \Omega_{h}} \frac{1}{2}\left|\left(\operatorname{div}_{h} \mathbf{s}\right)_{i, j}+f_{i, j}\right|^{2}+\frac{q_{n}}{2}\left|\mathbf{s}_{i, j}-\mathbf{t}_{i, j}\right|^{2}+\delta_{\mathcal{R}_{h}}(\mathbf{t}),
\end{aligned}
$$

where $\mathcal{R}_{h}=\left\{\mathbf{t} \in \mathbb{R}^{2 N^{2}}|| \mathbf{t}_{i, j} \mid \leq \alpha,(i, j) \in \Omega_{h}\right\}$, and the discrete indicator function is defined by

$$
\delta_{\mathcal{R}_{h}}(\mathbf{t})= \begin{cases}0 & \mathbf{t} \in \mathcal{R}_{h}, \\ +\infty & \text { otherwise }\end{cases}
$$

The discrete version of Algorithm 2 with $q=q_{n}>0$ is summarised in Algorithm 3 which generates a sequence $\left\{\left(\mathbf{s}_{n, h}^{k}, \mathbf{t}_{n, h}^{k}\right)\right\}_{k \in \mathbb{N}}$ with a fixed positive penalty parameter $q_{n}$ and $0<\eta<1$.

$\overline{\text { Algorithm } 3 \text { (Discrete pre-dual variable splitting with } \eta \text { - approx. discrete solution }}$ of $(20))$

1: Initialization. Choose $\mathbf{t}_{n, h}^{0} \in \mathcal{R}_{h}$ arbitrary and set $k:=1$.

2: Compute

$$
\mathbf{s}_{n, h}^{k} \in \underset{\mathbf{s}}{\arg \min } \sum_{(i, j) \in \Omega_{h}} \frac{1}{2}\left|\left(\operatorname{div}_{h} \mathbf{s}\right)_{i, j}+f_{i, j}\right|^{2}+\frac{q_{n}}{2}\left|\mathbf{s}_{i, j}-\eta\left(\mathbf{t}_{n, h}^{k-1}\right)_{i, j}\right|^{2} .
$$

3: Compute

$$
\mathbf{t}_{n, h}^{k} \in \underset{\mathbf{t}}{\arg \min } \sum_{(i, j) \in \Omega_{h}} \frac{q_{n}}{2}\left|\left(\mathbf{s}_{n, h}^{k}\right)_{i, j}-\mathbf{t}_{i, j}\right|^{2}+\delta_{\mathcal{R}_{h}}(\mathbf{t}) .
$$

4: Check stopping criteria. If suitable stopping criteria are met, set $\left(\mathbf{s}_{n, h}^{*}, \mathbf{t}_{n, h}^{*}\right)=$ $\left(\mathbf{s}_{n, h}^{k}, \mathbf{t}_{n, h}^{k}\right)$; otherwise set $k:=k+1$ are return to step 2 .

For a fixed $q_{n}$, suitable stopping criterion in step 4 of Algorithm 3 is considered satisfied when the norm of the residual of the first-order optimality conditions drops below a user-specified tolerance. The projection (27) provides an explicit formula for $\mathbf{t}_{n, h}^{k}$ in step 3, which satisfies the first-order condition of the $\mathbf{t}$-subproblem exactly. Thus, it is enough to check the residual of the first-order condition of the s-subproblem. For this purpose, we compute

$$
\mathbf{R}_{n, h}^{k}:=-\nabla_{h}\left(\operatorname{div}_{h} \mathbf{s}_{n, h}^{k}+f\right)+q_{n}\left(\mathbf{s}_{n, h}^{k}-\eta \mathbf{t}_{n, h}^{k}\right)
$$


and $\tilde{\mathbf{R}}_{n, h}^{k}$ such that $-\Delta_{h} \tilde{\mathbf{R}}_{n, h}^{k}=\mathbf{R}_{n, h}^{k}$ with homogeneous Dirichlet boundary conditions on $\partial \Omega_{h}$, where $\Delta_{h}$ denotes the discrete Laplacian on the underlying mesh. Then, the discrete $L^{2}(\Omega)$-norm of $\nabla_{h} \tilde{\mathbf{R}}_{n, h}^{k}$ provides the discrete $H^{-1}(\Omega)$-norm of $\mathbf{R}_{n, h}^{k}$. The value of $\mathbf{s}_{n, h}^{*}$ in step 4 of Algorithm 3 is $\mathbf{s}_{n, h}^{k}$ for the smallest $k$ that satisfies

$$
\left|\nabla_{h} \tilde{\mathbf{R}}_{n, h}^{k}\right|_{\mathbf{L}^{2}} \leq \epsilon_{d},
$$

where $\epsilon_{d}>0$ is some prescribed tolerance. The aforementioned index $k>0$ is the number of iterations of the algorithm and it is denoted as $K_{n, h}$, as it depends on the penalty parameter $q_{n}$ and the mesh size $h>0$. Starting with $q_{0}>0$, and increasing the penalty parameter up to $q_{n}$, the total number of iterations is the sum of the iterations corresponding to each penalty parameter, i.e.,

$$
K_{h}:=\sum_{i=0}^{n} K_{i, h}
$$

Finally, the approximated solution of the discrete TV-problem is determined as

$$
u_{n, h}^{*}=\operatorname{div}_{h} \mathbf{s}_{n, h}^{*}+f .
$$

As in [41] we use $q_{n+1}=2 q_{n}$ to increase the penalty parameters.

We also would like to emphasize the fact that our goal here is not to display the best possible reconstruction result, i.e., selecting optimal regularization parameters, but rather to focus on the algorithm and its behavior.

We start our numerical study by showing that the practical behavior of $\eta$ in (3) is consistent with Theorem 3.1. For this purpose, we use the image shown in Figure 1(a) which contains white Gaussian noise with zero mean and a standard deviation equal to 30. The parameters used in our algorithm are $\alpha=0.2, \epsilon_{d}=10^{-6}$ and the reconstruction is provided in Figure 1(b). Starting with a penalty parameter $q_{0}=2^{-5}$, we numerically compute $u_{n, h}^{*}$ for $n \in\{0,1, \ldots, 24\}$ with $h=1 / 128$. Since $u_{n, h}^{*}$ and $K_{h}$ depend on $\eta$, we stop our computations at $q_{25}=2^{20}$ for all $\eta$-values and denote these as $u_{\eta}$ and $K_{\eta}$, respectively. In order to measure more detailed features when $\eta$ approaches 1 , we choose $\eta_{m}$ according to

$$
\eta_{m}=\sum_{i=1}^{m} 9 \cdot 10^{-i} .
$$

Figure 1(c) shows the behavior of $\left|u_{\eta}-u_{\eta_{\infty}}\right|_{L^{2}}$ with respect to $\eta \in[0.1,1)$, where $\eta_{\infty}$ denotes the maximum $\eta$-value used which is $\eta_{9}=1-10^{-10}$, in this case. As we are in finite dimensions upon discretization, convergence of $u_{\eta}$ in the discrete $L^{2}(\Omega)$-norm of the approximated solution to the TV-problem in (40) is observed when $\eta \rightarrow 1$. This behavior is guaranteed by Theorem 3.1(ii.) considered in finite dimensions. Local Lipschitz behavior of $\eta \mapsto u_{\eta}$ is observed for $\eta<1$ and a non-Lipschitz behavior appears at $\eta=1$, as expected by Theorem 3.1(i.).

Figure 1(d) shows the behavior of $m \mapsto\left|u_{\eta_{m}}-u_{\eta_{\infty}-c}\right|_{L^{2}}$ with respect to $m$ as in (41) and with $c=10^{-3}$. This is consistent with the non-Lipschitz behavior of $\eta \mapsto u_{\eta}$ at $\eta=1$ expected from Theorem 3.1(i). The total number of iterations $K_{\eta_{m}}$ with respect to $m$ as in (41) is shown in Figure 1(e). The behavior of the map $m \mapsto K_{\eta_{m}}$ appears to contain two distinct features that are explained by the contraction of the map $\mathbf{s} \mapsto T\left(\eta P_{\mathcal{R}}(\mathbf{s})\right)$, as noted in the proof of Theorem 3.1, and 


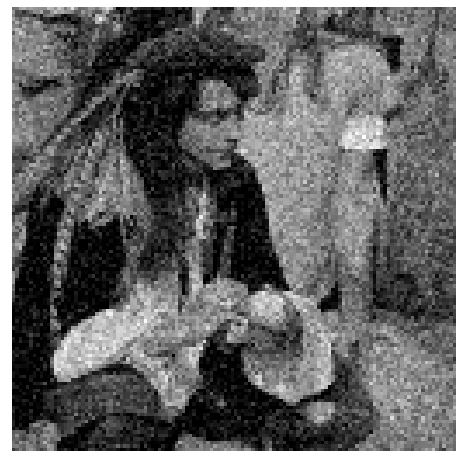

(a)

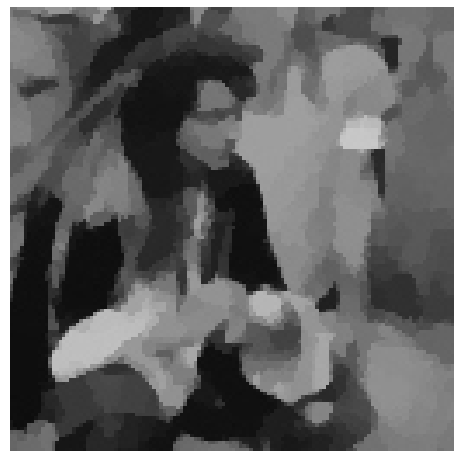

(b)

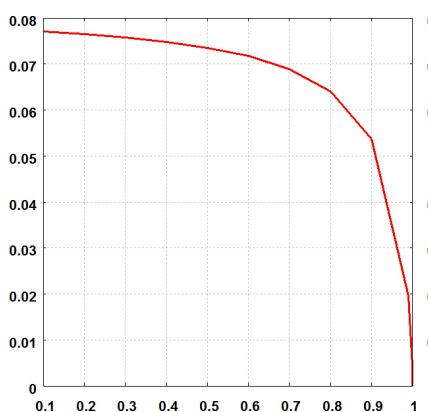

(c)

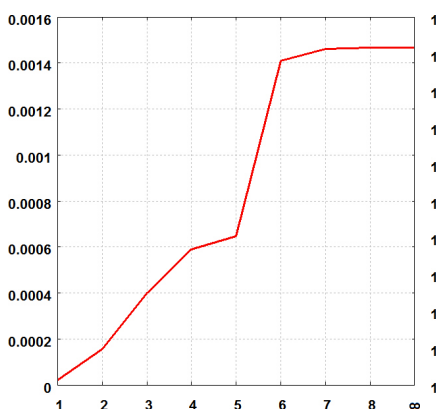

(d)

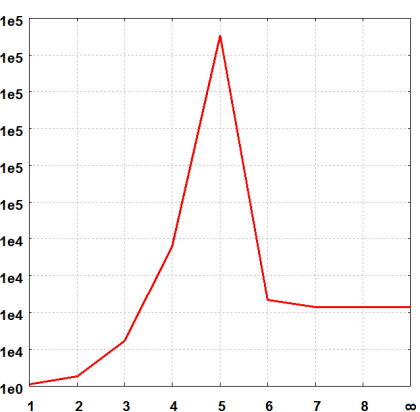

(e)

Figure 1: In figures (a) and (b) we depict the test image with white Gaussian noise of zero mean and standard deviation equal to 30 and its reconstruction with $\alpha=0.2$, respectively. In figure (c) we observe the behavior of the map $(0.1,1) \ni \eta \mapsto$ $\left|u_{\eta}-u_{\eta_{\infty}}\right|_{L^{2}}$, where $\eta_{\infty}=\eta_{9}=1-10^{-10}$. The $x$-axis in figures $1(\mathrm{~d}), 1(\mathrm{e})$ indicates the value $m$ in (41). In figure $1(\mathrm{~d})$, we observe $m \mapsto\left|u_{\eta_{m}}-u_{\eta_{m}-c}\right|_{L^{2}}$, where $c=10^{-3}$, and in Figure 1(e), the map $m \mapsto K_{\eta_{m}}$ (the total number of iterations associated with the parameter $\eta_{m}$ ) is shown.

the fact we are working in finite dimensions upon discretization. In fact, as noted before, step 2 of Algorithm 2 is equivalent to $\mathbf{s}_{\eta}^{k}=T\left(\eta P_{\mathcal{R}}\left(\mathbf{s}_{\eta}^{k-1}\right)\right)$. This iteration is at least linearly convergent since $\mathbf{s} \mapsto T\left(\eta P_{\mathcal{R}}(\mathbf{s})\right)$ is a contraction in the $q$-norm for $H_{0}$ (div), as observed in (26). For this purpose, recall that the $q$-norm is equivalent to the usual norm in $H_{0}$ (div). Since the Lipschitz constant is equal to $\eta$, it is expected that the increase of $\eta$ increases the number of iterations $K_{\eta}$ necessary to satisfy the small residual condition (38). This is indeed observed in Figure 1(e) for $1 \leq m \leq 5$. In infinite dimensions one would expect that this behavior continues for $m>5$. In finite dimensions, the weak convergence in Theorem 3.1(ii.) becomes strong convergence in the discrete $L^{2}(\Omega)$-norm, which appears to take place for $m>5$. This is the reason for the stabilization of the number of iterations necessary for the termination of the algorithm. An analogous reasoning applies to the behaviour of $m \mapsto\left|u_{\eta_{m}}-u_{\eta_{m}-c}\right|_{L^{2}}$ for $m>5$ in Figure 1(d).

It should be noted that the $L^{2}$-penalty term associated with the constraint $\mathbf{s}=\mathbf{t}$ in (20) does not increase the regularity of the solution as it happens with 
the penalization of the constraint $\mathbf{p}=\nabla u$ in (6). This enables the restoration of discontinuous features in images, as motivated by the total variation regularization.

3.3.2. Discrete augmented Lagrangian method for the pre-dual problem. We continue our investigation of the (pre-)dual problem by considering the discrete augmented Lagrangian associated with (30) given by

$$
\begin{aligned}
\mathcal{L}_{q, h}^{d}(\mathbf{s}, \mathbf{t} ; \boldsymbol{\mu}):=h^{2} \sum_{(i, j) \in \Omega_{h}} \frac{1}{2}\left|\left(\operatorname{div}_{h} \mathbf{s}\right)_{i, j}+f_{i, j}\right|^{2}+\frac{q}{2}\left|\mathbf{s}_{i, j}-\mathbf{t}_{i, j}\right|^{2} \\
+\boldsymbol{\mu}_{i, j} \cdot\left(\mathbf{s}_{i, j}-\mathbf{t}_{i, j}\right)+\delta_{\mathcal{R}_{h}}(\mathbf{t}) .
\end{aligned}
$$

For a fixed positive penalty parameter $q \geq 0$ we use an alternating direction minimization algorithm to generate a sequence $\left\{\left(\mathbf{s}_{q, h}^{k}, \mathbf{t}_{q, h}^{k}, \boldsymbol{\mu}_{q, h}^{k}\right)\right\}_{k \in \mathbb{N}}$ by Algorithm 4; see $[32,40]$ for details. Compared to (28a), we use here $\epsilon=0$.

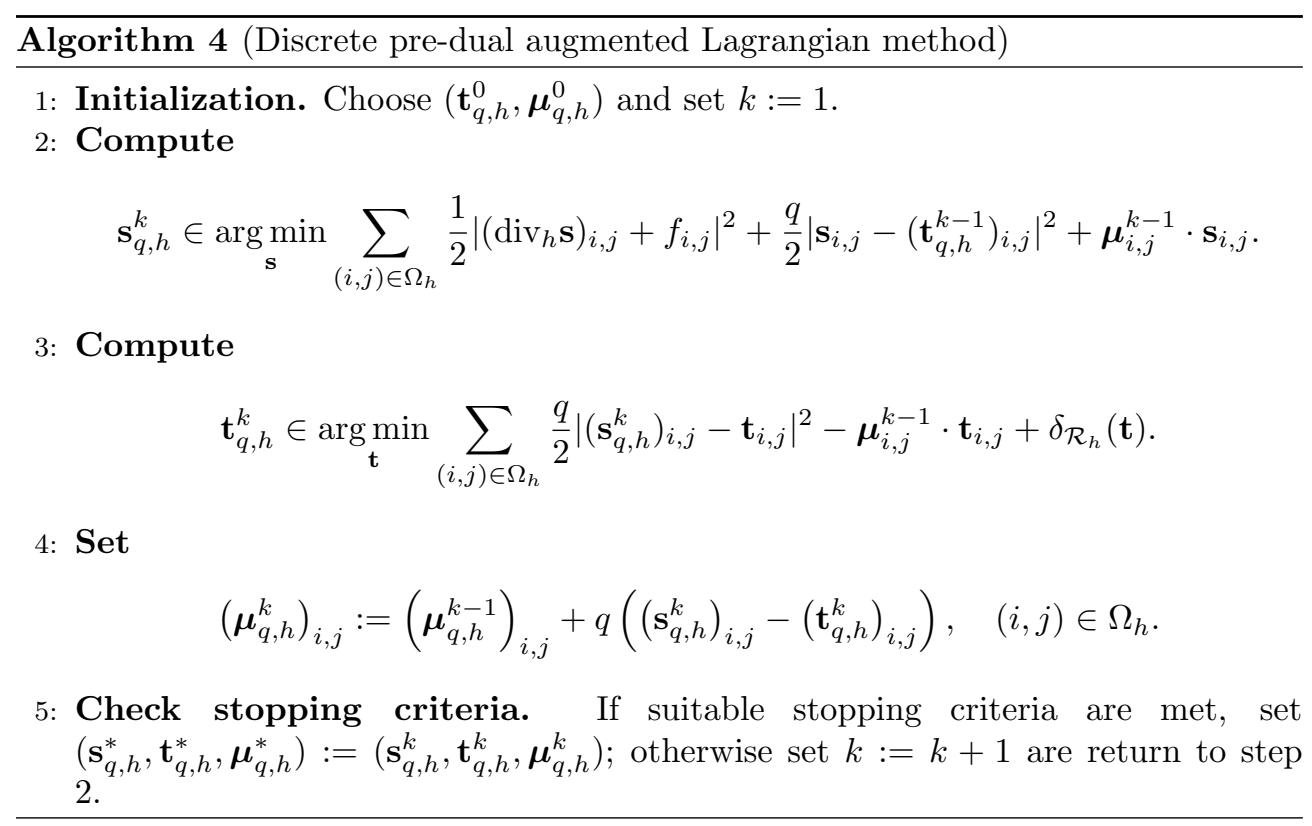

For a fixed $q$, as for the previous algorithms, suitable stopping criteria in step 5 of Algorithm 4 are based on the norm of the residuals of the first-order optimality conditions dropping below a user-specified tolerance. As observed before, a projectionlike technique gives an explicit formula for $\mathbf{t}_{q, h}^{k}$ in step 3. Hence, $\mathbf{t}_{q, h}^{k}$ satisfies the first-order condition for the $\mathbf{t}$-subproblem exactly. Thus, it is enough to check the residual of the first-order condition of the s-subproblem in the discrete analogue of $H^{-1}(\Omega)$. As before, we compute

$$
\mathbf{R}_{q, h}^{k}:=-\nabla_{h}\left(\operatorname{div}_{h} \mathbf{s}_{q, h}^{k}+f\right)+q\left(\mathbf{s}_{q, h}^{k}-\mathbf{t}_{q, h}^{k}\right)+\boldsymbol{\mu}_{q, h}^{k},
$$

find the solution of $-\Delta_{h} \tilde{\mathbf{R}}_{q, h}^{k}=\mathbf{R}_{q, h}^{k}$ with homogeneous Dirichlet boundary conditions on $\partial \Omega$ and otherwise proceed as in the previous subsection with some prescribed $\epsilon_{d}>0$ for the termination condition (38). 
Utilizing duality, an approximate solution to the discrete TV-problem is given by

$$
u_{q, h}^{*}=\operatorname{div}_{h} \mathbf{s}_{q, h}^{*}+f .
$$

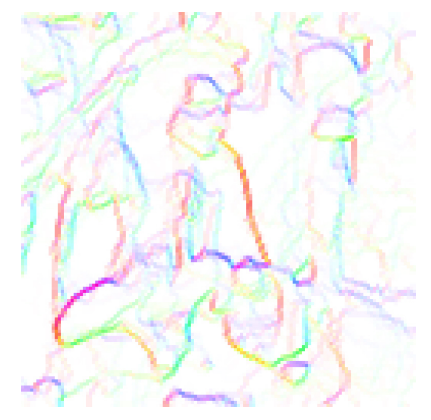

(a)

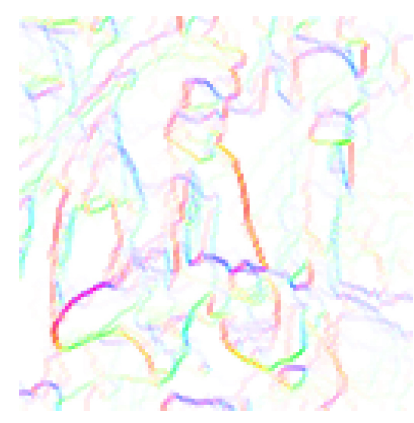

(b)

Figure 2: Figures (a) and (b) show the HSV-color-map, with $V=1$, for the vector fields $\boldsymbol{\mu}^{*}$ and $\nabla_{h} u^{*}$, respectively, as obtained by Algorithm 4. The original image is as in Figure 1(a)

A few words on the asymptotic behaviour of Algorithm 4 are in order; we specially concentrate on properties of $\boldsymbol{\mu}_{q, h}^{*}$. Suppose $\left\{\left(\mathbf{s}_{q, h}^{k}, \mathbf{t}_{q, h}^{k}, \boldsymbol{\mu}_{q, h}^{k}\right)\right\}_{k \in \mathbb{N}}$ is a sequence generated by Algorithm 4 and that it converges to $\left(\mathbf{s}^{*}, \mathbf{t}^{*}, \boldsymbol{\mu}^{*}\right):=\left(\mathbf{s}_{q, h}^{*}, \mathbf{t}_{q, h}^{*}, \boldsymbol{\mu}_{q, h}^{*}\right)$ with a fixed $q>0$ and $h$. Then the first-order optimality conditions associated with the steps of Algorithm 4 are given by

$$
\begin{gathered}
-\nabla_{h}\left(\operatorname{div}_{h} \mathbf{s}^{*}+f\right)+q\left(\mathbf{s}^{*}-\mathbf{t}^{*}\right)+\boldsymbol{\mu}^{*}=\mathbf{0} \\
\max \left\{\alpha,\left|\frac{\boldsymbol{\mu}^{*}}{q}+\mathbf{s}^{*}\right|\right\} \mathbf{t}^{*}=\alpha\left(\frac{\boldsymbol{\mu}^{*}}{q}+\mathbf{s}^{*}\right), \\
\mathbf{t}^{*}=\mathbf{s}^{*}
\end{gathered}
$$

Since $u^{*}=\operatorname{div}_{h} \mathbf{s}^{*}+f$, we have

$$
\boldsymbol{\mu}^{*}=\nabla_{h} u^{*}
$$

and further

$$
\max \left\{\alpha,\left|\mathbf{s}^{*}+\frac{\nabla_{h} u^{*}}{q}\right|\right\} \mathbf{s}^{*}=\alpha\left(\mathbf{s}^{*}+\frac{\nabla_{h} u^{*}}{q}\right) .
$$

It can be shown that $u^{*}$ and $\mathbf{s}^{*}$ satisfy the first-order optimality conditions (in finite dimensions) associated with the (pre-)dual formulation of the TV-problem (5). In the left plot of Figure 2 we depict the numerical solution $\boldsymbol{\mu}^{*}$, which was obtained with a very large penalty parameter $\left(q=2^{25}\right)$ and the same continuation technique as described in subsection 3.3.1. The regularization parameter was $\alpha=0.2, \epsilon_{d}=10^{-6}$ and we used the example in Figure 1(a). For the visualization we utilize an HSV-color space with $V=1$ to represent the vector fields of $\boldsymbol{\mu}^{*}$ and $\nabla_{h} u^{*}=\nabla_{h}\left(\operatorname{div}_{h} \mathbf{s}^{*}+f\right)$ in Figure 2(a) and Figure 2(b), respectively. This color map allows to represent vectors 
in an RGB color model, i.e., matching colors between the two plots correspond to matching vector orientations. From these plots we observe that $\boldsymbol{\mu}^{*}$ obtained from Algorithm 4 numerically converges indeed to $\nabla_{h} u^{*}$.

Unlike the penalty method in subsection 3.3.1 (which required certain limiting properties on $q_{n}$ and subsequently on $\eta$ ), the proposed algorithm in Algorithm 4 obtains an approximation to the solution of (16) without the need of driving the penalty $q>0$ parameter to infinity. Moreover, we numerically observe that Algorithm 4 achieves the stopping condition $\left|\nabla_{h} \tilde{\mathbf{R}}_{n, h}^{k}\right|_{\mathbf{L}^{2}} \leq \epsilon_{d}$ earlier than Algorithm 3 when the same penalty parameter is fixed in both algorithms . Note, however, that the previous assertions hold only in this finite dimensional setting and little is known about the infinite dimensional analogue of Algorithm 4 and its convergence behaviour.

\section{Staggered grid discretization for the pre-dual problem and numerical results}

In this section, we provide details of our discretization of the first-order optimality system associated with (20) or (30) on a staggered grid. Moreover, we include numerical evidence of the advantages of using such a staggered grid to solve the proposed systems and we compare with results obtained by other algorithms.

Considering the finite element method, Raviart-Thomas elements on a rectangular mesh are an appropriate choice for discretizing the spaces for minimizing the objectives in (20) and (30); see [17] for a specification of the Raviart-Thomas finite element space. This is due to the resulting discrete space being a finite dimensional subspace of $H_{0}$ (div). Here, we propose to use finite difference operators on a staggered grid. This is inspired by the fact that the lowest order Raviart-Thomas space on a rectangular mesh is equivalent to the "marker and cell" scheme (see [22]) which is a stable finite difference scheme for incompressible flow problems (see [26]).

\subsection{Finite difference operators on a staggered grid}

We consider the domain $\Omega=(0,1) \times(0,1)$ and the mesh size $h=1 / N$ for a positive integer $N \geq 2$. Figure 3(a) depicts an example for a grid associated with a $4 \times 4$ pixel image, i.e., $N=4$. The outermost boundary is the boundary of the image $\partial \Omega$. For the subsequent discretization of the involved operators we define the index sets

$$
\begin{aligned}
\Omega_{h}^{(a, b)}:= & \{(i, j) \in \mathbb{N} \times \mathbb{N} \mid 1 \leq i \leq N-a, 1 \leq j \leq N-b\}, \\
& \text { with }(a, b) \in\{(1,0),(0,0),(0,1)\}, \\
\bar{\Omega}_{h}^{(0,0)}:= & \Omega_{h}^{(0,0)} \cup\{(i, 0) \text { or }(i, N+1) \mid 1 \leq i \leq N\} \cup\{(0, j) \text { or }(N+1, j) \mid 1 \leq j \leq N\}, \\
\bar{\Omega}_{h}^{(1,0)}:= & \Omega_{h}^{(1,0)} \cup\{(0, j) \text { or }(N, j) \mid 1 \leq j \leq N\}, \\
\bar{\Omega}_{h}^{(0,1)}:= & \Omega_{h}^{(0,1)} \cup\{(i, 0) \text { or }(i, N) \mid 1 \leq i \leq N\} .
\end{aligned}
$$

The sets $\Omega_{h}^{(0,0)}, \Omega_{h}^{(1,0)}$ and $\Omega_{h}^{(0,1)}$ are identified with the set of $\bullet$-nodes, o-nodes, and $\square$-nodes in Figure 3, respectively. The set $\bar{\Omega}_{h}^{(0,0)}$ is identified with the •-nodes together with all the boundary nodes, $\bar{\Omega}_{h}^{(1,0)}$ with the o-nodes in addition to the nodes 
on the vertical boundary and $\bar{\Omega}_{h}^{(0,1)}$ with the $\square$-nodes and the nodes on the horizontal boundary, again depicted in Figure 3.

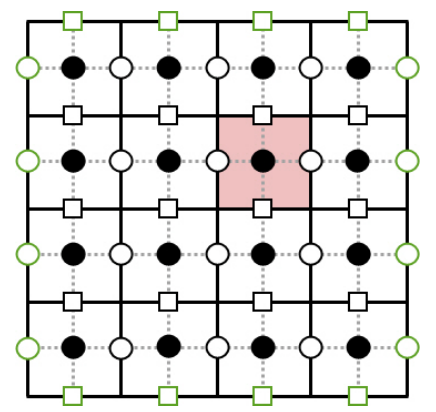

(a)

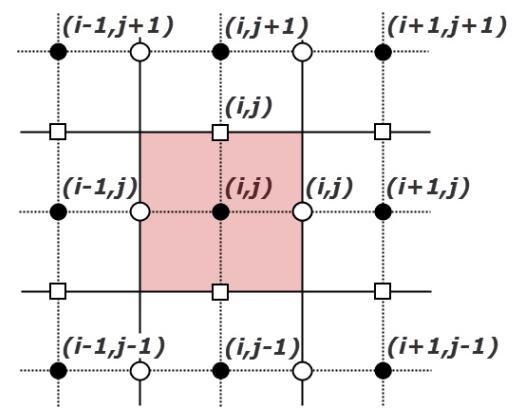

(b)

Figure 3: An example of a staggered grid for an image of size is $4 \times 4$. In (a) we depict $\Omega=(0,1) \times(0,1)$ with a discrete grid of mesh size $h=1 / 4$. Note that the Green nodes are exactly placed on the boundary $\partial \Omega$ of the domain $\Omega$. In (b) a rule of indexing for different variables in (20) and (29) is depicted.

We define sets of real-valued functions on a discrete domain by

$$
\mathcal{F}\left(\Omega_{h}^{(a, b)}\right):=\left\{f \mid f: \Omega_{h}^{(a, b)} \rightarrow \mathbb{R}\right\} .
$$

Since the mesh size $h$ is fixed, we simply write

$$
\mathcal{F}^{(a, b)}:=\mathcal{F}\left(\Omega_{h}^{(a, b)}\right) \quad \text { and } \quad \overline{\mathcal{F}}^{(a, b)}:=\mathcal{F}\left(\bar{\Omega}_{h}^{(a, b)}\right) .
$$

Note that following the identification of the sets $\Omega_{h}^{(a, b)}$ and $\bar{\Omega}_{h}^{(a, b)}$ with their corresponding nodes, we identify $\mathcal{F}^{(0,0)}, \mathcal{F}^{(1,0)}$, and $\mathcal{F}^{(0,1)}$ with the set of real-valued functions defined on the --nodes, the o-nodes, and the $\square$-nodes, respectively. Similar and analogous identifications are considered on the sets $\overline{\mathcal{F}}^{(a, b)}$.

Now, we define two finite difference operators on a staggered grid: the discrete divergence

$$
\operatorname{div}_{h}: \overline{\mathcal{F}}^{(1,0)} \times \overline{\mathcal{F}}^{(0,1)} \rightarrow \mathcal{F}^{(0,0)} \quad \text { by } \quad \operatorname{div}_{h} \mathbf{s}:=\partial_{1}^{-} s_{1}+\partial_{2}^{-} s_{2} \in \mathcal{F}^{(0,0)},
$$

and the discrete gradient

$$
\nabla_{h}: \mathcal{F}^{(0,0)} \rightarrow \mathcal{F}^{(1,0)} \times \mathcal{F}^{(0,1)} \quad \text { by } \quad \nabla_{h} u:=\left(\partial_{1}^{+} u, \partial_{2}^{+} u\right) \in \mathcal{F}^{(1,0)} \times \mathcal{F}^{(0,1)} .
$$

The above definitions include the standard finite forward and backward difference operators defined by

$$
\left(\partial_{1}^{ \pm} f\right)_{i, j}= \pm \frac{f_{i \pm 1, j}-f_{i, j}}{h} \quad \text { and } \quad\left(\partial_{2}^{ \pm} f\right)_{i, j}= \pm \frac{f_{i, j \pm 1}-f_{i, j}}{h} .
$$

The standard finite central difference operators are defined by

$$
\left(\partial_{1} f\right)_{i, j}=\frac{f_{i+1, j}-f_{i-1, j}}{2 h} \text { and }\left(\partial_{2} f\right)_{i, j}=\frac{f_{i, j+1}-f_{i, j-1}}{2 h} .
$$


Even though the discrete divergence $\operatorname{div}_{h}$ and gradient $\nabla_{h}$ are defined by standard finite forward and backward operators, they can be interpreted as a central difference scheme because of the structure of the node distribution on the staggered grid.

\subsection{Compatibility of adjoint operators and boundary condition}

We recall that the solution space of the Fenchel pre-dual problem (16) is $H_{0}$ (div). On a staggered grid the discrete version of $H_{0}($ div) becomes

$$
H_{0}^{\mathcal{F}}(\text { div }):=\left\{\begin{array}{l|l}
\left(s_{1}, s_{2}\right) \in \overline{\mathcal{F}}^{(1,0)} \times \overline{\mathcal{F}}^{(0,1)} & \begin{array}{l}
\left(s_{1}\right)_{i, j}=0,(i, j) \in \bar{\Omega}_{h}^{(1,0)} \backslash \Omega_{h}^{(1,0)} \\
\left(s_{2}\right)_{i, j}=0,(i, j) \in \bar{\Omega}_{h}^{(0,1)} \backslash \Omega_{h}^{(0,1)}
\end{array}
\end{array}\right\} .
$$

For this space, it can be shown that the discrete divergence (50) and gradient (51) are adjoint to each other under appropriate conditions. Indeed, for $\mathbf{s} \in H_{0}^{\mathcal{F}}$ (div) and $u \in \mathcal{F}^{(0,0)}$ we have

$$
\begin{aligned}
\left\langle u,-\operatorname{div}_{h} \mathbf{s}\right\rangle_{\mathcal{F}^{(0,0)}} & =\sum_{(i, j) \in \Omega_{h}^{(0,0)}} u_{i, j}\left(-\operatorname{div}_{h} \mathbf{s}\right)_{i, j} \\
& =\sum_{(i, j) \in \Omega_{h}^{(1,0)}}\left(s_{1}\right)_{i, j}\left(\partial_{1}^{+} u\right)_{i, j}+\sum_{(i, j) \in \Omega_{h}^{(0,1)}}\left(s_{2}\right)_{i, j}\left(\partial_{2}^{+} u\right)_{i, j} \\
& =\left\langle\mathbf{s}, \nabla_{h} u\right\rangle_{\mathcal{F}^{(1,0)} \times \mathcal{F}^{(0,1)}},
\end{aligned}
$$

where the last equality considers $\mathbf{s}$ restricted to $\Omega_{h}^{(1,0)} \times \Omega_{h}^{(0,1)}$. Note that (54) might not hold for arbitrary $\mathbf{s} \in \overline{\mathcal{F}}^{(1,0)} \times \overline{\mathcal{F}}^{(0,1)} \backslash H_{0}^{\mathcal{F}}($ div $)$.

The discrete divergence and gradient oprerators introduced by Chambolle in [8] are formally of the same form as the ones in (50) and (51), respectively. However, it should be noted that for the discrete operators in [8], evaluations for all variables are computed on the same nodes. Hence, in order to obtain that the discrete gradient is the adjoint of the negative discrete divergence, additional boundary conditions for the primal variable are required.

\subsection{Numerical realization of our algorithms}

We now provide details on the numerical implementation of Algorithm 2 on a staggered grid (the extension of the method for the minimization of (30) with $\tilde{\mathcal{R}}$ and $|\cdot|_{H_{0}(\text { div })}$ replaced by $\mathcal{R}$ and $|\cdot|_{L^{2}}$, respectively, is straightforward). Note that, in these settings, the discrete functionals in (35) and (42) are typical discretizations of (20) and (30), respectively, when all variables are evaluated on the $\bullet$-nodes; see Figure 3.

The variables $\bar{u}, f, \mathbf{s}, \mathbf{t}$ and $\boldsymbol{\lambda}$ in (17), (35) and (42) associated to discrete spaces are as follows:

$$
\bar{u}, f \in \mathcal{F}^{(0,0)}, \quad \mathbf{s} \in H_{0}^{\mathcal{F}}(\operatorname{div}), \quad \mathbf{t}, \boldsymbol{\lambda} \in \mathcal{F}^{(1,0)} \times \mathcal{F}^{(0,1)} .
$$

Since the first and second components of a vector field on a staggered grid are evaluated at different locations, we need an interpolation operator whenever a function value is needed at an alternative location. Here, we use a simple linear interpolation. In fact, 
for given $\mathbf{s} \in \mathcal{F}^{(1,0)} \times \mathcal{F}^{(0,1)}$, the following averaging operators are used:

$\mathcal{A}\left(s_{2}\right)_{i, j}=\frac{\left(s_{2}\right)_{i, j}+\left(s_{2}\right)_{i+1, j}+\left(s_{2}\right)_{i, j-1}+\left(s_{2}\right)_{i+1, j-1}}{4}, \quad$ for $(i, j) \in \Omega_{h}^{(1,0)}, s_{2} \in \overline{\mathcal{F}}^{(0,1)}$,

$\mathcal{A}\left(s_{1}\right)_{i, j}=\frac{\left(s_{1}\right)_{i, j}+\left(s_{1}\right)_{i, j+1}+\left(s_{1}\right)_{i-1, j}+\left(s_{1}\right)_{i-1, j+1}}{4}, \quad$ for $(i, j) \in \Omega_{h}^{(0,1)}, s_{1} \in \overline{\mathcal{F}}^{(1,0)}$.

Consequently and depending on the nodes, the absolute values of $\mathbf{s} \in \mathcal{F}^{(1,0)} \times \mathcal{F}^{(0,1)}$ are defined by

$$
\begin{aligned}
& \left|\mathbf{s}_{i, j}\right|^{2}=\left(s_{1}\right)_{i, j}^{2}+\mathcal{A}\left(s_{2}\right)_{i, j}^{2}, \quad \text { for }(i, j) \in \Omega_{h}^{(1,0)} \\
& \left|\mathbf{s}_{i, j}\right|^{2}=\mathcal{A}\left(s_{1}\right)_{i, j}^{2}+\left(s_{2}\right)_{i, j}^{2}, \quad \text { for }(i, j) \in \Omega_{h}^{(0,1)} \\
& \left|\mathbf{s}_{i, j}\right|^{2}=\left(\frac{\left(s_{1}\right)_{i, j}+\left(s_{1}\right)_{i-1, j}}{2}\right)^{2}+\left(\frac{\left(s_{2}\right)_{i, j}+\left(s_{2}\right)_{i, j-1}}{2}\right)^{2}, \text { for }(i, j) \in \Omega_{h}^{(0,0)} .
\end{aligned}
$$

It should be noted that the above definitions are consistent as the mesh size tends to zero under a continuity assumption: In fact, for $(i, j) \in \Omega_{h}^{(a, b)}$ with $(a, b) \in\{(1,0),(0,0),(0,1)\}$ let $s_{1}, s_{2} \in C(\bar{\Omega} ; \mathbb{R})$ and $\left(s_{k}\right)_{i, j}$ correspond to the pointwise evaluation of $s_{k}$ on the corresponding node. Then the relations (58)-(60) hold as $h \downarrow 0$. This yields the desired inequality $|\mathbf{s}(\mathbf{x})| \leq \alpha$ for all $\mathbf{x} \in \Omega$.

Suppose $q_{n}>0$ and $0<\eta<1$ are fixed and $\mathbf{t}_{\eta}^{0}$ is given. Denote by $\left\{\left(\mathbf{s}_{n}^{k}, \mathbf{t}_{n}^{k}\right)\right\}_{k \in \mathbb{N}}$ the sequence generated by Algorithm 2. According to first-order optimality, $\mathbf{s}_{n}^{k}$ solves the system

$$
\begin{aligned}
-\nabla(\operatorname{divs}+f)+q_{n}\left(\mathbf{s}-\eta \mathbf{t}_{n}^{k-1}\right) & =\mathbf{0} & & \text { in } \Omega, \\
\mathbf{s} \cdot \nu & =0 & & \text { on } \partial \Omega,
\end{aligned}
$$

where $\nu$ denotes the outward unit normal on $\partial \Omega$, and $\mathbf{t}_{n}^{k}$ is obtained in closed form by

$$
\mathbf{t}_{n}^{k}(\mathbf{x})= \begin{cases}\mathbf{s}_{n}^{k}(\mathbf{x}), & \text { if }\left|\mathbf{s}_{n}^{k}(\mathbf{x})\right| \leq \alpha, \mathbf{x} \in \Omega \\ \frac{\mathbf{s}_{n}^{k}(\mathbf{x})}{\left|\mathbf{s}_{n}^{k}(\mathbf{x})\right|}, & \text { if }\left|\mathbf{s}_{n}^{k}(\mathbf{x})\right|>\alpha, \quad \mathbf{x} \in \Omega\end{cases}
$$

Considering the staggered grid in Figure 3, we solve the two subproblems in Algorithm 2 by using the discrete operators (50) and (51). For a fixed mesh size $h$, the solution $\mathbf{s}_{n, h}^{k} \in H_{0}^{\mathcal{F}}$ (div) of the discrete s-subproblem is obtained by solving

$$
\begin{aligned}
-\partial_{1}^{+}\left(\left(\partial_{1}^{-} s_{1}\right)_{i, j}+\left(\partial_{2}^{-} s_{2}\right)_{i, j}\right)+q_{n}\left(s_{1}\right)_{i, j} & =\left(\partial_{1}^{+} f\right)_{i, j}+q_{n} \eta\left(\left(t_{1}\right)_{n, h}^{k-1}\right)_{i, j}, \quad(i, j) \in \Omega_{h}^{(1,0)}, \\
-\partial_{2}^{+}\left(\left(\partial_{1}^{-} s_{1}\right)_{i, j}+\left(\partial_{2}^{-} s_{2}\right)_{i, j}\right)+q_{n}\left(s_{2}\right)_{i, j} & =\left(\partial_{2}^{+} f\right)_{i, j}+q_{n} \eta\left(\left(t_{2}\right)_{n, h}^{k-1}\right)_{i, j}, \quad(i, j) \in \Omega_{h}^{(0,1)}, \\
\left(s_{1}\right)_{i, j} & =0, \quad(i, j) \in \bar{\Omega}_{h}^{(1,0)} \backslash \Omega_{h}^{(1,0)}, \\
\left(s_{2}\right)_{i, j} & =0, \quad(i, j) \in \bar{\Omega}_{h}^{(0,1)} \backslash \Omega_{h}^{(0,1)} .
\end{aligned}
$$


Further, $\mathbf{t}_{n, h}^{k} \in \mathcal{F}^{(1,0)} \times \mathcal{F}^{(0,1)}$ is computed as

$$
\begin{aligned}
& \left(\left(t_{1}\right)_{n, h}^{k}\right)_{i, j}=\left\{\begin{array}{lll}
\left(\left(s_{1}\right)_{n, h}^{k}\right)_{i, j}, & \text { if }\left|\mathbf{s}_{n, h}^{k}\right|_{i, j} \leq \alpha, & (i, j) \in \Omega_{h}^{(1,0)}, \\
\frac{\left(\left(s_{1}\right)_{n}^{k}\right)_{i, j}}{\left|\mathbf{s}_{n, h}^{k}\right|_{i, j}}, & \text { if }\left|\mathbf{s}_{n, h}^{k}\right|_{i, j}>\alpha, & (i, j) \in \Omega_{h}^{(1,0)},
\end{array}\right. \\
& \left(\left(t_{2}\right)_{n, h}^{k}\right)_{i, j}=\left\{\begin{array}{lll}
\left(\left(s_{2}\right)_{n, h}^{k}\right)_{i, j}, & \text { if }\left|\mathbf{s}_{n, h}^{k}\right|_{i, j} \leq \alpha, & (i, j) \in \Omega_{h}^{(0,1)}, \\
\frac{\left(\left(s_{2}\right)_{n, h}^{k}\right)_{i, j}}{\left|\mathbf{s}_{n, h}^{k}\right|_{i, j}}, & \text { if }\left|\mathbf{s}_{n, h}^{k}\right|_{i, j}>\alpha, & (i, j) \in \Omega_{h}^{(0,1)}
\end{array}\right.
\end{aligned}
$$

As the solution in $\mathbf{t}$ is exact, the iteration along $k$ is stopped when the discrete residual of the optimality condition for the s-subproblem is sufficiently small. In fact, since the equation in the first line of (61) holds in $H^{-1}(\Omega)$, a special step is require to measure the residual. For this purpose, after we extend $\mathbf{t}_{n, h}^{k} \in \mathcal{F}^{(1,0)} \times \mathcal{F}^{(0,1)}$ to $H_{0}^{\mathcal{F}}$ (div) and $f \in \mathcal{F}^{(0,0)}$ to $\overline{\mathcal{F}}^{(0,0)}$ by homogeneous Neumann boundary conditions on $\partial \Omega$, we compute the vector field

$$
\mathbf{R}_{n, h}^{k}:=-\nabla_{h}\left(\operatorname{div}_{h} \mathbf{s}_{n, h}^{k}+f\right)+q_{n}\left(\mathbf{s}_{n, h}^{k}-\eta \mathbf{t}_{n, h}^{k}\right) \in \overline{\mathcal{F}}^{(1,0)} \times \overline{\mathcal{F}}^{(0,1)},
$$

and find $\tilde{\mathbf{R}}_{n, h}^{k}=\left(\left(\tilde{R}_{1}\right)_{n, h}^{k},\left(\tilde{R}_{2}\right)_{n, h}^{k}\right) \in \overline{\mathcal{F}}^{(0,0)} \times \overline{\mathcal{F}}^{(0,0)}$ as the solution of

$$
\begin{array}{cc}
-\left(\left(\partial_{1}^{+} \partial_{1}^{-}+\partial_{2}^{+} \partial_{2}^{-}\right)\left(\tilde{R}_{l}\right)_{n, h}^{k}\right)_{i, j}=\frac{1}{2}\left(\left(\left(R_{l}\right)_{n, h}^{k}\right)_{i, j}+\left(\left(R_{l}\right)_{n, h}^{k}\right)_{i-(2-l), j-(l-1)}\right), & (i, j) \in \begin{array}{c}
\Omega_{h}^{(0,0)}, \\
(66)
\end{array} \\
\left(\left(\tilde{R}_{l}\right)_{n, h}^{k}\right)_{i, j}=0, \quad(i, j) \in \bar{\Omega}_{h}^{(0,0)} \backslash \Omega_{h}^{(0,0)}, & (67)
\end{array}
$$

for $l \in\{1,2\}$. The numerical solution, $\mathbf{s}_{n, h}^{*}:=\mathbf{s}_{n, h}^{k}$, is obtained for the smallest $k \geq 1$ such that

$$
\left|\nabla_{h} \tilde{\mathbf{R}}_{n, h}^{k}\right|_{\mathbf{L}^{2}} \approx\left(h^{2} \sum_{\substack{l \in\{1,2\} \\(i, j) \in \Omega_{h}^{(0,0)}}}\left|\left(\partial_{1}\left(\tilde{R}_{l}\right)_{n, h}^{k}\right)_{i, j}\right|^{2}+\left|\left(\partial_{2}\left(\tilde{R}_{l}\right)_{n, h}^{k}\right)_{i, j}\right|^{2}\right)^{\frac{1}{2}} \leq \epsilon_{d},
$$

for a prescribed stopping tolerance $\epsilon_{d}>0$.

The approximate solution of the discrete TV-problem is then given by

$$
\left(u_{n, h}^{*}\right)_{i, j}=\left(\operatorname{div}_{h} \mathbf{s}_{n, h}^{*}\right)_{i, j}+f_{i, j}, \quad(i, j) \in \Omega_{h}^{(0,0)} .
$$

For increasing $q_{n}$, we use the same continuation scheme as described before.

\subsection{Numerical Results}

Next we compare our results numerically with those obtained from different algorithms. In all examples below the respective image intensity is scaled to $[0,1]$. For simplicity we use the following abbreviations for the methods of Sections 2 and 3 :

- $P_{A L M}$ : Primal augmented Lagrangian method of subsection 2.1.1.

- $D_{A L M}$ : The augmented Lagrangian method of the Fenchel pre-dual formulation in subsection 3.3.2. 


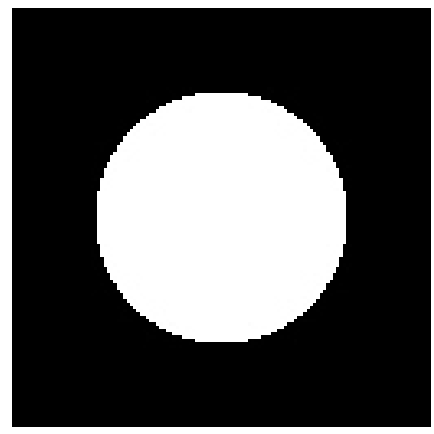

(a)

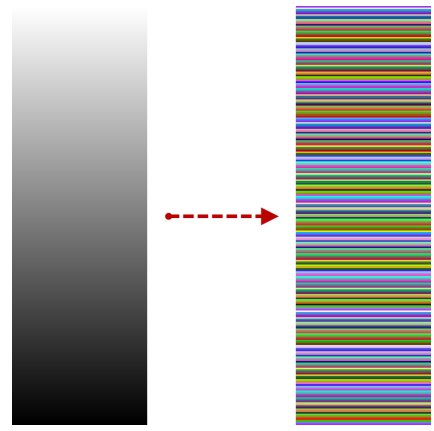

(d)

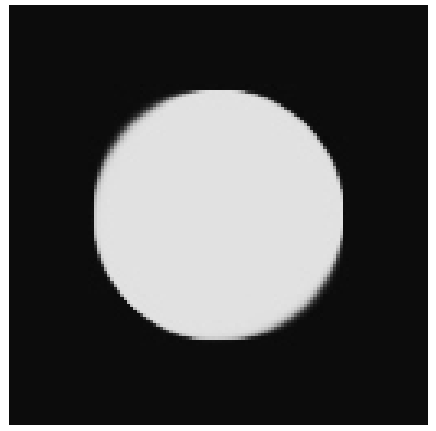

(b)

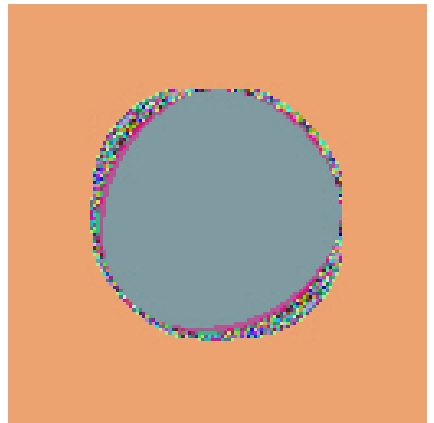

(e)

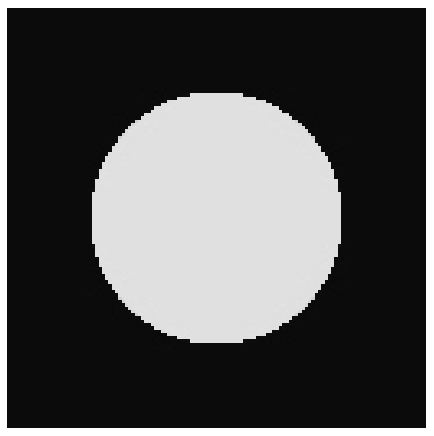

(c)

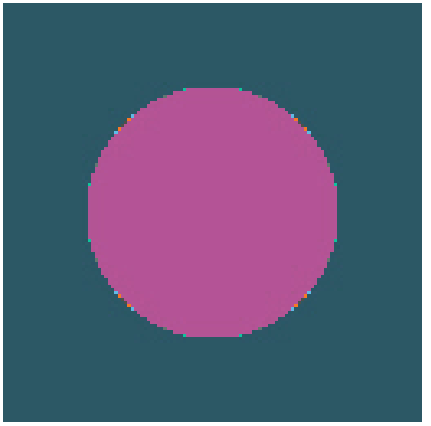

(f)

Figure 4: A qualitative comparison between $P_{A L M}$ and $D_{A L M}$ : (a) The $128 \times 128$ image $f$ for the TV-problem. The results from $P_{A L M}$ and $D_{A L M}$ are shown in (b) and (c), respectively. The color table to change a gray-scale image into a color image is shown in (d). In (e) and (f), we show the correspondingly colored versions of the images in (b) and (c), respectively. It is easy to observe that $D_{A L M}$ generates an almost piecewise constant image.

Note that we use the same discretization as in [42] for $P_{A L M}$. Unless otherwise specified, we use the previously described discretization on the staggered grid of subsection 4.1 for $D_{A L M}$.

First, we provide a qualitative comparison between $P_{A L M}$ and $D_{A L M}$ in Figure 4 . In order to ensure a fair comparison, we choose an example with

$$
f=\chi_{\mathcal{C}}, \quad \mathcal{C}:=\{\mathbf{x} \in(0,1) \times(0,1):|\mathbf{x}-(0.5,0.5)| \leq 0.3\}
$$

with known exact solution; see [38] or [39]. Figure 4(a) shows the $128 \times 128$ image $f$ in the TV-problem . For $P_{A L M}, \epsilon_{p}=10^{-2}, r_{0}=2^{-10}$ and $r_{n+1}=2 r_{n}$ are used. Figure 4(b) displays the numerical solution obtained by $P_{A L M}$ with penalty parameter $r_{22}=2^{12}$. For $D_{A L M}, \epsilon_{d}=10^{-6}, q_{0}=2^{10}$ and $q_{n+1}=2 q_{n}$ are used. Figure 4(c) shows the numerical solution obtained by $D_{A L M}$ with penalty parameter $q_{15}=2^{25}$. We use the same idea as proposed in [9] to change a gray-scale image into a color image based on the color table in Figure 4(d). Figure 4(e) and Figure 4(f) are the 
color images corresponding to Figure 4(b) and Figure 4(c), respectively, based on the aforementioned color table.

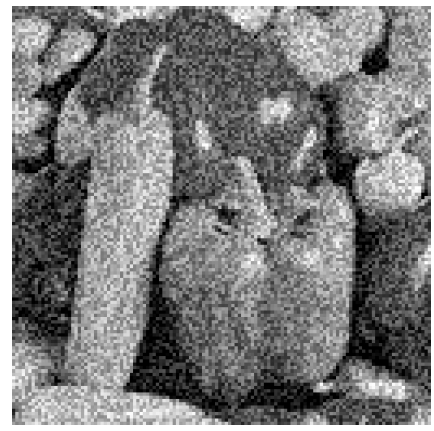

(a)

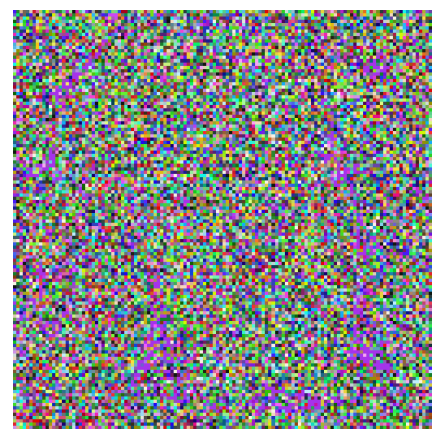

(d)

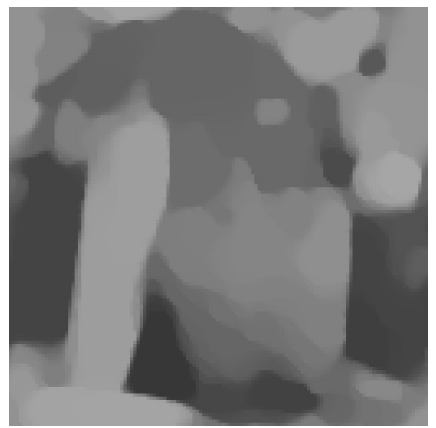

(b)

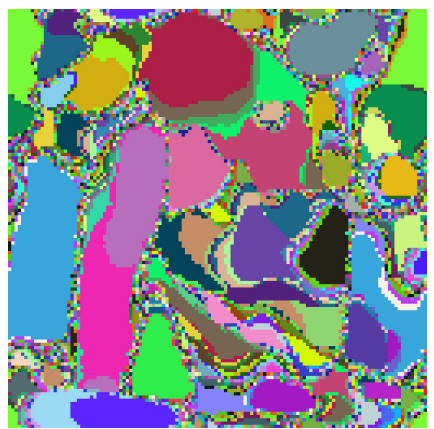

(e)

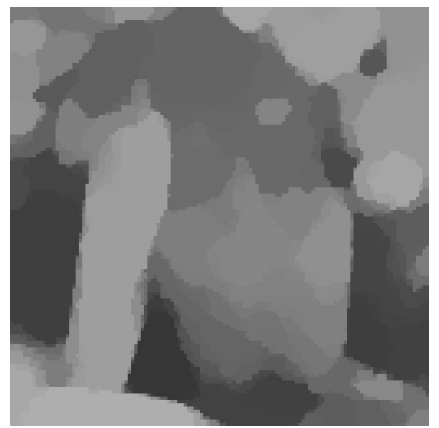

(c)

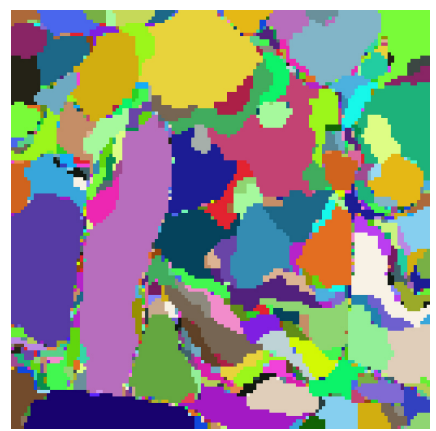

(f)

Figure 5: A qualitative comparison between $P_{A L M}$ and $D_{A L M}$ : (a) The $128 \times 128$ image $f$ in the TV-problem . The results for $P_{A L M}$ and $D_{A L M}$ can be seen in (b) and (c), respectively. Figures (d), (e), and (f) are the corresponding color images to (a), (b) and (c), respectively, based on a color table in Figure 4(d).

We readily observe that the results of $P_{A L M}$ in Figures $4(\mathrm{~b})$ and $4(\mathrm{e})$ suffer from blurriness depending on radial directions from the center of the circle. The direction dependent error is caused by the one-sided discretization scheme in [42]. It is significantly reduced by using the upwind finite-difference method in [9]. The errors responsible for blurry regions in the reconstruction cannot be overcome by the algorithms in Section 2 as the solution space in case of a finite penalty parameter is $W^{1,1}(\Omega)$. The latter does not admit edge structures. On the other hand, the Fenchel pre-dual formulation is crucially different in this respect. The numerical result from $D_{A L M}$ seems to approximate a piece-wise constant function with the exception of a small number of pixels (compare, for example, with the results obtained in [9, Figure 5.1]). We also note that the one-sided discretization scheme of [42] for the algorithms in Section 3 generate a result similar to Figure 4(b).

For a further detailed and exhaustive study on the anisotropy induced by discretization methods and their connection with infinite dimensional models we refer 
to $[28]$.

In Figure 5, the original image is degraded by Gaussian white noise with a standard deviation of 20 . Figure 5 (a) shows the $128 \times 128$ noisy image $f$ in the TVproblem. A relatively large regularization parameter $\alpha=1$ is used, as we primarily want to study discretization aspects. In this respect, recovering "too" many detailed features in Figure 5(c) would render the comparison of effects at boundaries between constant image features impossible. For $P_{A L M}, \epsilon_{p}=10^{-2}, r_{0}=2^{-10}$ and $r_{n+1}=2 r_{n}$ are used. Figure 5(b) depicts the numerical solution obtained by $P_{A L M}$ when the penalty parameter is $r_{30}=2^{20}$. For $D_{A L M}, \epsilon_{d}=10^{-6}, q_{0}=2^{10}$ and $q_{n+1}=2 q_{n}$ are used. Figure $5(\mathrm{c})$ depicts the numerical solution obtained by $D_{A L M}$ when the penalty parameter reaches $q_{15}=2^{25}$. Figures $5(\mathrm{~d}), 5(\mathrm{e})$, and $5(\mathrm{f})$ are the color images corresponding to $5(\mathrm{a}), 5(\mathrm{~b})$, and $5(\mathrm{c})$, respectively, based on the color table in Figure 4(d). We observe a similar behavior as in Figure 4. The numerical result from $D_{A L M}$ is piecewise constant which is supposed to be a solution of the TV-problem, whereas the non-staggered grid is responsible for blurring effects at boundaries between contour pieces.

\section{Conclusion}

While variable splitting methods have been used successfully in image processing over the recent years, usually with the understanding that they solve a discrete version of, e.g., the TV-problem, the situation for the original, genuinely infinite dimensional TV-problem is different.

Primal variable splitting techniques are in general not well-defined as the existence of a solution of the associated penalty problem cannot be guaranteed. Rather it can be shown that for increasing penalty parameters, $\epsilon$-minimizers (in the sense of Ekeland's variational principle) converge to the solution of the TV-problem. Nevertheless, the alternating iteration schemes, often utilized for solving the penalty problem, appear functional, as the associated subproblems admit (unique) solutions. Their convergence, obviously, is jeopardized by the possible non-existence of solutions of the associated minimization problem.

The situation for the pre-dual problem (note that the dual would lead to a problem in the dual of the non-reflexive space of functions of bounded variation) is entirely different: (i) Variable splitting techniques are well-defined in the sense that associated penalty or augmented Lagrangian based problems admit solutions in function space; (ii) the pre-dual is posed in Hilbert space; and (iii) the associated alternating minimization, Bregman splitting or augmented Lagrangian methods exhibit guaranteed convergence properties and perform better than their primal counterparts (upon discretization).

Finally, as the pre-dual is posed in $H_{0}$ (div), staggered grids are appropriate for discretization as they provided stable (and numerically significantly less diffusive and somewhat isotropic) finite difference schemes. In this paper this was validated

numerically including a comparison with other specialized discretization schemes for dual formulations of the discrete TV-problem.

\section{Appendix A. Appendix : Proofs of Theorems 2.1 and 2.2}

Proof. [Theorem 2.1] We split the proof into several steps. 
Step 1: The sequence $\left\{u_{n}\right\}$ is bounded in $B V(\Omega)$. By the definition of $\left\{\left(u_{n}, \mathbf{p}_{n}\right)\right\}$ in (7) we have $\mathcal{E}_{n}^{p}\left(u_{n}, \mathbf{p}_{n}\right) \leq \mathcal{E}_{n}^{p}(0,0)+\epsilon_{n}=\frac{1}{2} \int_{\Omega}|f|^{2}+\epsilon_{n}$. Thus, $\left\{\frac{r_{n}}{2}\left|\mathbf{p}_{n}-\nabla u_{n}\right|_{\mathbf{L}^{2}(\Omega)}^{2}\right\}$ is bounded. Also, $r_{n} \uparrow \infty$ and hence $\left|\mathbf{p}_{n}-\nabla u_{n}\right|_{\mathbf{L}^{2}(\Omega)} \rightarrow 0$ as $n \rightarrow \infty$ and, by Hölder's and the triangle inequalities, $\delta_{n}:=\alpha \int_{\Omega}\left|\mathbf{p}_{n}\right|-\alpha \int_{\Omega}\left|\nabla u_{n}\right|$ satisfies $\lim _{n \rightarrow \infty} \delta_{n}=0$.

From the definition of $\delta_{n}$ and $\left(u_{n}, \mathbf{p}_{n}\right)$, we obtain

$$
\begin{aligned}
-\delta_{n}+\frac{1}{2} \int_{\Omega}|f|^{2} & =\alpha\left(\int_{\Omega}\left|\nabla u_{n}\right|-\int_{\Omega}\left|\mathbf{p}_{n}\right|\right)+\frac{1}{2} \int_{\Omega}|f|^{2} \\
& \geq \alpha\left(\int_{\Omega}\left|\nabla u_{n}\right|-\int_{\Omega}\left|\mathbf{p}_{n}\right|\right)+\mathcal{E}_{n}^{p}\left(u_{n}, \mathbf{p}_{n}\right)-\epsilon_{n} \\
& =\alpha \int_{\Omega}\left|\nabla u_{n}\right|+\frac{1}{2} \int_{\Omega}\left|u_{n}-f\right|^{2}+\frac{r_{n}}{2}\left|\mathbf{p}_{n}-\nabla u_{n}\right|_{\mathbf{L}^{2}}^{2}-\epsilon_{n} \\
& \geq \alpha \int_{\Omega}\left|\nabla u_{n}\right|+\frac{1}{2|\Omega|}\left(\int_{\Omega}\left|u_{n}-f\right|\right)^{2}+\frac{r_{n}}{2}\left|\mathbf{p}_{n}-\nabla u_{n}\right|_{\mathbf{L}^{2}}^{2}-\epsilon_{n} \\
& \geq \alpha \int_{\Omega}\left|\nabla u_{n}\right|+\frac{1}{2|\Omega|}\left(\int_{\Omega}\left|u_{n}\right|-\int_{\Omega}|f|\right)^{2}+\frac{r_{n}}{2}\left|\mathbf{p}_{n}-\nabla u_{n}\right|_{\mathbf{L}^{2}}^{2}-\epsilon_{n},
\end{aligned}
$$

where Hölder's inequality yields (A.4) and the reverse triangle inequality (A.5).

Since $\delta_{n} \rightarrow 0$ and $\epsilon_{n} \downarrow 0$, we find that the sequences

$$
\left\{\int_{\Omega}\left|u_{n}\right|\right\} \quad \text { and } \quad\left\{\int_{\Omega}\left|\mathcal{D} u_{n}\right|\right\}
$$

are bounded (note that $\int_{\Omega}\left|\mathcal{D} u_{n}\right|=\int_{\Omega}\left|\nabla u_{n}\right|$ for each $n$, since $u_{n} \in W^{1,1}(\Omega)$ ), i.e., $\left\{u_{n}\right\}$ is bounded in $B V(\Omega)$.

Step 2: $\underline{\lim }_{n \rightarrow \infty} r_{n}\left|\mathbf{p}_{n}-\nabla u_{n}\right|_{L^{2}}^{2}=0$. From the definition of $\left\{\left(u_{n}, \mathbf{p}_{n}\right)\right\}$ in (7), we have that $\mathcal{E}_{n}^{p}\left(u_{n}, \mathbf{p}_{n}\right) \leq \mathcal{E}_{n}^{p}(u, \nabla u)+\epsilon_{n}$ for any $u \in W^{1,1}(\Omega)$, i.e.,

$$
\frac{1}{2} \int_{\Omega}\left|u_{n}-f\right|^{2}+\alpha \int_{\Omega}\left|\mathbf{p}_{n}\right|+\frac{r_{n}}{2}\left|\mathbf{p}_{n}-\nabla u_{n}\right|_{\mathbf{L}^{2}}^{2} \leq \frac{1}{2} \int_{\Omega}|u-f|^{2}+\alpha \int_{\Omega}|\nabla u|+\epsilon_{n} .
$$

From the definition of $\delta_{n}$, we get $\alpha \int_{\Omega}\left|\mathbf{p}_{n}\right|=\delta_{n}+\alpha \int_{\Omega}\left|\nabla u_{n}\right|$. Since $\left\{u_{n}\right\}$ is bounded in $B V(\Omega)$ and, thus, in $L^{2}(\Omega)$ (recall that $B V(\Omega) \hookrightarrow L^{2}(\Omega)$; see [2]) there is $\bar{u} \in B V(\Omega)$ for which $u_{n} \rightarrow \bar{u}$ in $L^{2}(\Omega)$, along a subsequence. Since the embedding $B V(\Omega) \hookrightarrow L^{1}(\Omega)$ is compact (see [19]), we observe that $u_{n} \rightarrow \bar{u}$ in $L^{1}(\Omega)$ (along a subsequence of $\left\{u_{n}\right\}$ which we also denote by $\left\{u_{n}\right\}$ ). This implies $\int_{\Omega}|\mathcal{D} \bar{u}| \leq \underline{\lim }_{n \rightarrow \infty} \int_{\Omega}\left|\nabla u_{n}\right|$. Thus, we have $\int_{\Omega}|\mathcal{D} \bar{u}| \leq \underline{\lim }_{n \rightarrow \infty} \int_{\Omega}\left|\mathbf{p}_{n}\right|$ and therefore

$$
\begin{aligned}
& \frac{1}{2} \int_{\Omega}|\bar{u}-f|^{2}+\alpha \int_{\Omega}|\mathcal{D} \bar{u}|+a \leq \\
& \underline{\lim _{n \rightarrow \infty}}\left(\frac{1}{2} \int_{\Omega}\left|u_{n}-f\right|^{2}+\alpha \int_{\Omega}\left|\mathbf{p}_{n}\right|+\frac{r_{n}}{2}\left|\mathbf{p}_{n}-\nabla u_{n}\right|_{\mathbf{L}^{2}}^{2}\right),
\end{aligned}
$$

where $a=\underline{\lim }_{n \rightarrow \infty} \frac{r_{n}}{2}\left|\mathbf{p}_{n}-\nabla u_{n}\right|_{\mathbf{L}^{2}}^{2}$. Here we have used the weak lower semicontinuity of the norm and elementary properties of lim. Therefore, from (A.7), we infer

$$
0 \leq \frac{1}{2} \int_{\Omega}|\bar{u}-f|^{2}+\alpha \int_{\Omega}|\mathcal{D} \bar{u}|+a \leq \frac{1}{2} \int_{\Omega}|u-f|^{2}+\alpha \int_{\Omega}|\nabla u|,
$$


for all $u \in W^{1,1}(\Omega)$.

Since $\bar{u} \in B V(\Omega) \cap L^{2}(\Omega)$, one can argue the existence of $\left\{v_{k}\right\} \subset C^{\infty}(\bar{\Omega}) \subset$ $W^{1,1}(\Omega)$ such that $\lim _{k \rightarrow \infty}\left|v_{k}-\bar{u}\right|_{L^{2}}=0$ and $\lim _{k \rightarrow \infty} \int_{\Omega}\left|\nabla v_{k}\right|=\int_{\Omega}|\mathcal{D} \bar{u}|$ (see for example [7]). We choose $u=v_{k}$ in the inequality (A.9) and take the limit as $k \rightarrow \infty$ to obtain $a=\underline{\lim }_{n \rightarrow \infty} \frac{r_{n}}{2}\left|\mathbf{p}_{n}-\nabla u_{n}\right|_{L^{2}(\Omega)}^{2}=0$.

Step 3: $\bar{u} \in B V(\Omega)$ is the minimizer. Finally, since $a=0$ in (A.9), we have that

$$
\frac{1}{2} \int_{\Omega}|\bar{u}-f|^{2}+\alpha \int_{\Omega}|\mathcal{D} \bar{u}| \leq \frac{1}{2} \int_{\Omega}|w-f|^{2}+\alpha \int_{\Omega}|\mathcal{D} w|
$$

for all $w \in B V(\Omega)$ by using the same density argument as in the preceding paragraph, i.e., $\bar{u}$ is a minimizer of the TV-problem .

Step 4: $u_{n} \rightarrow \bar{u}$ in the $L^{2}(\Omega)$-sense and $\int_{\Omega}\left|\mathbf{p}_{n}\right| \rightarrow \int_{\Omega}|\mathcal{D} \bar{u}|$ (along subsequences). Using $a=\underline{\lim }_{n \rightarrow \infty} \frac{r_{n}}{2}\left|\mathbf{p}_{n}-\nabla u_{n}\right|_{\mathbf{L}^{2}}^{2}=0$ and the inequalities in (A.7) and (A.8), we obtain that

$$
\varliminf_{n \rightarrow \infty}\left(\frac{1}{2} \int_{\Omega}\left|u_{n}-f\right|^{2}+\alpha \int_{\Omega}\left|\mathbf{p}_{n}\right|\right)=\frac{1}{2} \int_{\Omega}|\bar{u}-f|^{2}+\alpha \int_{\Omega}|\mathcal{D} \bar{u}|,
$$

where the density argument of Step 3 has been used. Then, we consider a subsequence of $\left\{\left(u_{n}, \mathbf{p}_{n}\right)\right\}$ (again denoted by $\left\{\left(u_{n}, \mathbf{p}_{n}\right)\right\}$ ) for which the previous relation holds when "lim" is replaced by "lim". From the previous steps, we know that $\int_{\Omega}|\bar{u}-f|^{2} \leq$ $\underline{\lim }_{n \rightarrow \infty} \int_{\Omega}\left|u_{n}-f\right|^{2}$, due to $u_{n} \rightarrow \bar{u}$ in the $L^{2}(\Omega)$-sense, and $\int_{\Omega}|\mathcal{D} \bar{u}| \leq \underline{\lim }_{n \rightarrow \infty} \int_{\Omega}\left|\mathbf{p}_{n}\right|$. Hence, we have $-\underline{\lim }_{n \rightarrow \infty} \int_{\Omega}\left|\mathbf{p}_{n}\right| \leq-\int_{\Omega}|\mathcal{D} \bar{u}|$ and equivalently $\varlimsup_{n \rightarrow \infty} \int_{\Omega}\left(-\left|\mathbf{p}_{n}\right|\right) \leq$ $-\int_{\Omega}|\mathcal{D} \bar{u}|$. Therefore, we obtain

$$
\begin{aligned}
\frac{1}{2} \int_{\Omega}|\bar{u}-f|^{2} & \leq \varliminf_{n \rightarrow \infty} \frac{1}{2} \int_{\Omega}\left|u_{n}-f\right|^{2} \\
& =\varliminf_{n \rightarrow \infty}\left(\frac{1}{2} \int_{\Omega}\left|u_{n}-f\right|^{2}+\alpha \int_{\Omega}\left|\mathbf{p}_{n}\right|-\alpha \int_{\Omega}\left|\mathbf{p}_{n}\right|\right) \\
& \leq \varlimsup_{n \rightarrow \infty}\left(\frac{1}{2} \int_{\Omega}\left|u_{n}-f\right|^{2}+\alpha \int_{\Omega}\left|\mathbf{p}_{n}\right|-\alpha \int_{\Omega}\left|\mathbf{p}_{n}\right|\right) \\
& \leq \frac{1}{2} \int_{\Omega}|\bar{u}-f|^{2}+\alpha \int_{\Omega}|\mathcal{D} \bar{u}|+\varlimsup_{n \rightarrow \infty}\left(-\alpha \int_{\Omega}\left|\mathbf{p}_{n}\right|\right) \\
& \leq \frac{1}{2} \int_{\Omega}|\bar{u}-f|^{2}+\alpha \int_{\Omega}|\mathcal{D} \bar{u}|-\alpha \int_{\Omega}|\mathcal{D} \bar{u}| \\
& =\frac{1}{2} \int_{\Omega}|\bar{u}-f|^{2} .
\end{aligned}
$$

Hence, $\underline{\lim }_{n \rightarrow \infty} \int_{\Omega}\left|u_{n}-f\right|^{2}=\int_{\Omega}|\bar{u}-f|^{2}$, and along a subsequence of $\left\{u_{n}\right\}$ we have $\int_{\Omega}\left|u_{n}-f\right|^{2} \rightarrow \int_{\Omega}|\bar{u}-f|^{2}$. We also have $\left(u_{n}-f\right) \rightarrow(\bar{u}-f)$ in $L^{2}(\Omega)$. Therefore, as $L^{2}(\Omega)$ is a locally uniformly convex space, $\left(u_{n}-f\right) \rightarrow(\bar{u}-f)$ in $L^{2}(\Omega)$ and hence $\left|u_{n}-\bar{u}\right|_{L^{2}} \rightarrow 0$ along a subsequence.

Finally, since we have

$$
\lim _{n \rightarrow \infty}\left(\frac{1}{2} \int_{\Omega}\left|u_{n}-f\right|^{2}+\alpha \int_{\Omega}\left|\mathbf{p}_{n}\right|\right)=\frac{1}{2} \int_{\Omega}|\bar{u}-f|^{2}+\alpha \int_{\Omega}|\mathcal{D} \bar{u}|,
$$

and $\left(u_{n}-f\right) \rightarrow(\bar{u}-f)$ in $L^{2}(\Omega)$ along a subsequence of $\left\{\left(u_{n}, \mathbf{p}_{n}\right)\right\}$, we have that $\lim _{n \rightarrow \infty} \int_{\Omega}\left|\mathbf{p}_{n}\right|=\int_{\Omega}|\mathcal{D} \bar{u}|$. 
Proof. [Theorem 2.2] The proof is split into two steps.

Step 1: The sequence $\left\{u_{n}\right\}$ is bounded in $B V(\Omega)$. By the definition of $\left\{\left(u_{n}, \mathbf{p}_{n}\right)\right\}$ we have $\mathcal{L}_{r_{n}}^{p}\left(u_{n}, \mathbf{p}_{n}, \boldsymbol{\lambda}_{n}\right) \leq \mathcal{L}_{r_{n}}^{p}\left(0,0, \boldsymbol{\lambda}_{n}\right)+\epsilon_{n}$. Then, upon completing squares, we find that $r_{n}\left|\mathbf{p}_{n}-\nabla u_{n}\right|_{\mathbf{L}^{2}}^{2}$ is bounded by (12). This implies $\lim _{n \rightarrow \infty}\left|\mathbf{p}_{n}-\nabla u_{n}\right|_{\mathbf{L}^{2}}=0$ and $\lim _{n \rightarrow \infty}\left\langle\boldsymbol{\lambda}_{n}, \mathbf{p}_{n}-\nabla u_{n}\right\rangle_{\mathbf{L}^{2}}=0$. Then, arguing as in Step 1 of the proof of Theorem 2.1 , we obtain

$$
\begin{aligned}
& -\delta_{n}+\frac{1}{2} \int_{\Omega}|f|^{2} \geq \\
& \alpha \int_{\Omega}\left|\nabla u_{n}\right|+\frac{1}{2|\Omega|}\left(\int_{\Omega}\left|u_{n}\right|-\int_{\Omega}|f|\right)^{2}+\frac{r_{n}}{2}\left|\mathbf{p}_{n}-\nabla u_{n}\right|_{L^{2}(\Omega)}^{2}+\left\langle\boldsymbol{\lambda}_{n}, \mathbf{p}_{n}-\nabla u_{n}\right\rangle_{\mathbf{L}^{2}}-\epsilon_{n},
\end{aligned}
$$

where $\delta_{n}=\alpha\left(\int_{\Omega}\left|\mathbf{p}_{n}\right|-\int_{\Omega}\left|\nabla u_{n}\right|\right)$. The latter expression tends to zero as $n \rightarrow \infty$ since $\lim _{n \rightarrow \infty}\left|\mathbf{p}_{n}-\nabla u_{n}\right|_{\mathbf{L}^{2}}=0$. Hence, as $\epsilon_{n} \downarrow 0$ we have that the sequences

$$
\left\{\int_{\Omega}\left|u_{n}\right|\right\} \text { and }\left\{\int_{\Omega}\left|\mathcal{D} u_{n}\right|\right\}
$$

are bounded. Thus, we conclude that $\left\{u_{n}\right\}$ in $W^{1,1}(\Omega)$ is bounded in $B V(\Omega)$.

Step 2: $\varliminf_{n \rightarrow \infty} r_{n}\left|\mathbf{p}_{n}-\nabla u_{n}\right|_{L^{2}}^{2}=0$, existence of a minimizer and appropriate convergence. From the definition of $\left\{\left(u_{n}, \mathbf{p}_{n}\right)\right\}$, we infer $\mathcal{L}_{r_{n}}^{p}\left(u_{n}, \mathbf{p}_{n}, \boldsymbol{\lambda}_{n}\right) \leq$ $\mathcal{L}_{r_{n}}^{p}\left(u, \nabla u, \boldsymbol{\lambda}_{n}\right)+\epsilon_{n}$ for any $u \in W^{1,1}(\Omega)$. Since we know that $\lim _{n \rightarrow \infty}\left\langle\boldsymbol{\lambda}_{n}, \mathbf{p}_{n}-\right.$ $\left.\nabla u_{n}\right\rangle_{\mathbf{L}^{2}}=0$, we argue as in the Step 2 of the proof of Theorem 2.1 and obtain a subsequence of $\left\{u_{n}\right\}$ ( also denoted by $\left\{u_{n}\right\}$ ) and $\bar{u} \in B V(\Omega)$ such that $u_{n} \rightarrow \bar{u}$ in $L^{2}(\Omega)$ and $\int_{\Omega}|\mathcal{D} u| \leq \underline{\lim }_{n \rightarrow \infty} \int_{\Omega}\left|\nabla u_{n}\right|$. Then, $\int_{\Omega}|\mathcal{D} u| \leq \underline{\lim }_{n \rightarrow \infty} \int_{\Omega}\left|\mathbf{p}_{n}\right|$ due to $\delta_{n}=\alpha\left(\int_{\Omega}\left|\mathbf{p}_{n}\right|-\int_{\Omega}\left|\nabla u_{n}\right|\right)$ going to 0 as $n \rightarrow \infty$, and we observe

$$
0 \leq \frac{1}{2} \int_{\Omega}|\bar{u}-f|^{2}+\alpha \int_{\Omega}|\mathcal{D} \bar{u}|+a \leq \frac{1}{2} \int_{\Omega}|u-f|^{2}+\alpha \int_{\Omega}|\nabla u|,
$$

where $a=\varliminf_{n \rightarrow \infty} \frac{r_{n}}{2}\left|\mathbf{p}_{n}-\nabla u_{n}\right|_{L^{2}(\Omega)}^{2}$. A density argument analogous to the one at the end of Step 2 and Step 3 of the proof of Theorem 2.1 implies that $a=0$ and consequently that $\bar{u}$ is a minimizer of the TV- problem . Finally, Step 4 in the proof of Theorem 2.1 is valid here and the assertion follows.

\section{References}

[1] R. A. Adams and J. J. F. Fournier. Sobolev Spaces. Academic Press, second edition, 2003.

[2] L. Ambrosio, N. Fusco, and D. Pallara. Functions of Bounded Variation and Free Discontinuity Problems. Oxford Mathematical Monographs, 2000.

[3] H. Attouch, G. Buttazzo, and G. Michaille. Variational Analysis in Sobolev and BV Spaces. MPS-SIAM, 2006.

[4] H. Brézis. Opérateurs maximaux monotones et semi-groupes de contractions dans les espaces de Hilbert. North-Holland Publishing Co., Amsterdam, 1973. North-Holland Mathematics Studies, No. 5. Notas de Matemática (50).

[5] R. E. Bruck, Jr. An iterative solution of a variational inequality for certain monotone operators in Hilbert space. Bull. Amer. Math. Soc., 81(5):890-892, 1975.

[6] R. E. Bruck, Jr. Corrigendum: "An iterative solution of a variational inequality for certain monotone operators in Hilbert space" (Bull. Amer. Math. Soc. 81 (1975), no. 5, 890-892). Bull. Amer. Math. Soc., 82(2):353, 1976.

[7] E. Casas, K. Kunisch, and C. Pola. Regularization by functions of bounded variation and applications to image enhancement. Applied Mathematics and Optimization, 40:229-257, 1999. 
[8] A. Chambolle. An algorithm for total variational minimization and applications. J. Math. Imaging Vis., 20:89-97, 2004.

[9] A. Chambolle, S.E. Levine, and B.J. Lucier. An upwind finite-difference method for total variation-based image smoothing. SIAM J. Imaging Sci., 4:277-299, 2011.

[10] R. Chan, M. Tao, and X. Yuan. Constrained total variation deblurring models and fast algorithms based on alternating direction method of multipliers. SIAM Journal on Imaging Sciences, 6(1):680-697, 2013.

[11] T.F. Chan, G.H. Golub, and P. Mulet. A nonlinear primal-dual method for total variation-based image restoration. SIAM J. Sci. Comput., 20:1964-1977, 1999.

[12] G. Hong-Gang Chen. Forward-backward splitting techniques: Theory and applications. ProQuest LLC, Ann Arbor, MI, 1994. Thesis (Ph.D.)-University of Washington.

[13] K. Chen, Y. Dong, and M. Hintermüller. A nonlinear multigrid solver with line GaussSeidel-semismooth-Newton smoother for the Fenchel pre-dual in total variation based image restoration. Inverse Problems and Imaging, 5(2):323-339, 2011.

[14] P. Chen, J. Huang, and X. Zhang. A primal-dual fixed point algorithm for convex separable minimization with applications to image restoration. Inverse Problems, 29(2):025011, 2013.

[15] Y. Chen, W. Hager, F. Huang, D. Phan, X. Ye, and W. Yin. Fast algorithms for image reconstruction with application to partially parallel $\mathrm{mr}$ imaging. SIAM Journal on Imaging Sciences, 5(1):90-118, 2012.

[16] J. Eckstein. Splitting methods for monotone operators with applications to parallel optimization. $\mathrm{PhD}$ thesis, Massachusetts Institute of Technology. Dept. of Civil Engineering., 1989.

[17] A. Ern and J.L. Guermond. Theory and Practice of Finite Elements. Number v. 159 in Applied Mathematical Sciences. Springer, 2004.

[18] D. Gabay. Applications of the method of multipliers to variational inequalities. In M. Fortin and R. Glowinski, editors, Augmented Lagrange Methods: Applications to the Solution of Boundary-valued Problems. North-Holland, Amsterdam, Netherlands, 1983.

[19] E. Giusti. Minimal Surfaces and Functions of Bounded Variation. Birkhäuser, 1984.

[20] A. A. Goldstein. Convex programming in Hilbert space. Bull. Amer. Math. Soc., 70:709-710, 1964.

[21] T. Goldstein and S. Osher. The split Bregman method for L1-regularized problems. SIAM J. Img. Sci., 2:323-343, 2009.

[22] F. H. Harlow and J. E. Welch. Numerical calculation of time-dependent viscous incompressible flow of fluid with free surface. Phys. Fluids, 8(12):2182-2189, 1965.

[23] M. Hintermüller and K. Kunisch. Total bounded variation regularization as a bilaterally constrained optimization problem. SIAM Journal of Applied Mathematics, 64:1311-1333, 2004.

[24] M. Hintermüller and G. Stadler. An infeasible primal-dual algorithm for total bounded variationbased inf-convolution-type image restoration. SIAM Journal of Scientific Computing, 28:1$23,2006$.

[25] K. Ito and K. Kunisch. An active set strategy based on the augmented Lagrangian formulation for image restoration. ESAIM: Mathematical Modelling and Numerical Analysis, 33:1-21, 1999.

[26] G. Kanschat. Divergence-free discontinuous Galerkin schemes for the Stokes equations and the MAC scheme. Int. J. Numer. Methods Fluids, 56(7):941-950, 2008.

[27] S. Kurcyusz. On the existence and nonenxistence of Lagrange multipliers in banach spaces. Journal of Optimization Theory and Applications, 20(1):81-110, 1976.

[28] J. Lellmann, B. Lellmann, F. Widmann, and C. Schnörr. Discrete and continuous models for partitioning problems. Int. J. Comp. Vis, 104(3):241-269, 2013.

[29] P.-L. Lions. Une méthode itérative de résolution d'une inéquation variationnelle. Israel J. Math., 31(2):204-208, 1978.

[30] P.-L. Lions and B. Mercier. Splitting algorithms for the sum of two nonlinear operators. SIAM J. Numer. Anal., 16(6):964-979, 1979.

[31] D. G. Luenberger. Optimization by Vector Space Methods. John Wiley \& Sons, 1969.

[32] M. Ng, P. Weiss, and X. Yuan. Solving constrained total-variation image restoration and reconstruction problems via alternating direction methods. SIAM Journal on Imaging Sciences, 32(5):2710-2736, 2010.

[33] Gregory B. Passty. Ergodic convergence to a zero of the sum of monotone operators in Hilbert space. J. Math. Anal. Appl., 72(2):383-390, 1979.

[34] R. R. Phelps. Lectures on maximal monotone operators. Extracta Math., 12(3):193-230, 1997.

[35] L. I. Rudin, S. Osher, and E. Fatemi. Nonlinear total variation based noise removal algorithms. Physica D, 60:259-268, 1992. 
[36] S. Setzer. Operator splittings, Bregman methods and frame shrinkage in image processing. Int. J. Comput. Vis, 92(3):265-280, 2011.

[37] R. E. Showalter. Monotone operators in Banach space and nonlinear partial differential equations, volume 49 of Mathematical Surveys and Monographs. American Mathematical Society, Providence, RI, 1997.

[38] D. M. Strong and T. F. Chan. Exact solutions to total variation regularization problems. Technical report, UCLA CAM Report, 1996.

[39] D. M. Strong and T. F. Chan. Edge-preserving and scale-dependent properties of total variation regularization. Inverse Problems, 19:165-187, 2003.

[40] X.-C. Tai and C. Wu. Augmented Lagrangian method, dual methods and split Bregman iteration for ROF model. In SSVM '09: Proceedings of the Second International Conference on Scale Space and Variational Methods in Computer Vision, pages 502-513, Berlin, Heidelberg, 2009. Springer-Verlag.

[41] Y. Wang, J. Yang, W. Yin, and Y. Zhang. A new alternating minimization algorithm for total variation image reconstruction. SIAM Journal on Imaging Sciences, 1:248-272, 2008.

[42] C. Wu and X.-C. Tai. Augmented Lagrangian method, dual methods, and split Bregman iteration for ROF, vectorial TV, and high order models. SIAM Journal on Imaging Sciences, 3(3):300-339, 2010

[43] J. Yang, W. Yin, Y. Zhang, and Y. Wang. A fast algorithm for edge-preserving variational multichannel image restoration. SIAM Journal on Imaging Sciences, 2(2):569-592, 2009.

[44] J. Zowe and S. Kurcyusz. Regularity and stability for the mathematical programming problem in Banach spaces. Applied Mathematics and Optimization, 5:49-62, 1979. 